\title{
Molecules, shocks, and disk in the axi-symmetric wind of the MS-type AGB star RS Cancri`
}

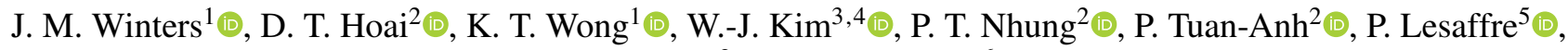 \\ P. Darriulat ${ }^{2}$, and T. Le Bertre ${ }^{6}(0)$ \\ ${ }^{1}$ Institut de Radioastronomie Millimétrique (IRAM), 300 rue de la Piscine, Domaine Universitaire, 38406 St. Martin d'Hères, France \\ e-mail: winters@iram.fr \\ ${ }^{2}$ Department of Astrophysics, Vietnam National Space Center (VNSC), Vietnam Academy of Science and Technology (VAST), \\ 18 Hoang Quoc Viet, Cau Giay, Ha Noi, Vietnam \\ ${ }^{3}$ Instituto de Radioastronomía Milimétrica (IRAM), Av. Divina Pastora 7, Núcleo Central, 18012, Granada, Spain \\ ${ }^{4}$ I. Physikalisches Institut, Universität zu Köln, Zülpicher Str. 77, 50937 Köln, Germany \\ ${ }^{5}$ Laboratoire de Physique de l'École Normale Supérieure, 24 rue Lhomond, 75231 Paris, France \\ ${ }^{6}$ LERMA, UMR 8112, CNRS and Observatoire de Paris, PSL Research University, 61 av. de l'Observatoire, 75014 Paris, France
}

Received 29 June 2021 / Accepted 29 November 2021

\begin{abstract}
Context. The latest evolutionary phases of low- and intermediate-mass stars are characterized by complex physical processes like turbulence, convection, stellar pulsations, magnetic fields, condensation of solid particles, and the formation of massive outflows that inject freshly produced heavy elements and dust particles into the interstellar medium.

Aims. By investigating individual objects in detail, we wish to analyze and disentangle the effects of the interrelated physical processes on the structure of the wind-forming regions around them.

Methods. We use the Northern Extended Millimeter Array to obtain spatially and spectrally resolved observations of the semiregular asymptotic giant branch (AGB) star RS Cancri and apply detailed 3D reconstruction modeling and local thermodynamic equilibrium radiative transfer calculations in order to shed light on the morpho-kinematic structure of its inner, wind-forming environment.

Results. We detect 32 lines of 13 molecules and isotopologs (CO, SiO, $\left.\mathrm{SO}, \mathrm{SO}_{2}, \mathrm{H}_{2} \mathrm{O}, \mathrm{HCN}, \mathrm{PN}\right)$, including several transitions from vibrationally excited states. $\mathrm{HCN}, \mathrm{H}^{13} \mathrm{CN}$, and millimeter vibrationally excited $\mathrm{H}_{2} \mathrm{O}, \mathrm{SO},{ }^{34} \mathrm{SO}, \mathrm{SO}_{2}$, and $\mathrm{PN}$ are detected for the first time in RS Cnc. Evidence for rotation is seen in $\mathrm{HCN}, \mathrm{SO}, \mathrm{SO}_{2}$, and $\mathrm{SiO}(v=1)$. From $\mathrm{CO}$ and $\mathrm{SiO}$ channel maps, we find an inner, equatorial density enhancement, and a bipolar outflow structure with a mass-loss rate of $1 \times 10^{-7} M_{\odot} \mathrm{yr}^{-1}$ for the equatorial region and of $2 \times 10^{-7} M_{\odot} \mathrm{yr}^{-1}$ for the polar outflows. The ${ }^{12} \mathrm{CO} /{ }^{13} \mathrm{CO}$ ratio is measured to be $\sim 20$ on average, $24 \pm 2$ in the polar outflows and $19 \pm 3$ in the equatorial region. We do not find direct evidence of a companion that might explain this kind of kinematic structure, and explore the possibility that a magnetic field might be the cause of it. The innermost molecular gas is influenced by stellar pulsation and possibly by convective cells that leave their imprint on broad wings of certain molecular lines, such as $\mathrm{SiO}$ and $\mathrm{SO}$.

Conclusions. RS Cnc is one of the few nearby, low-mass-loss-rate, oxygen-rich AGB stars with a wind displaying both an equatorial disk and bipolar outflows. Its orientation with respect to the line of sight is particularly favorable for a reliable study of its morphokinematics. Nevertheless, the mechanism causing early spherical symmetry breaking remains uncertain, calling for additional high spatial- and spectral-resolution observations of the emission of different molecules in different transitions, along with more thorough investigation of the coupling among the different physical processes at play.
\end{abstract}

Key words. stars: AGB and post-AGB - circumstellar matter - stars: mass-loss - stars: winds, outflows stars: individual: $\mathrm{RS} \mathrm{Cnc}$ - radio lines: stars

\section{Introduction}

Mass-loss in red giants is due to a combination of stellar pulsations and radiation pressure on dust forming in dense shocked regions in the outer stellar atmosphere (e.g., Höfner \& Olofsson 2018). Even if the basic principles are understood, a fully consistent picture - including the role of convection, the time-dependent chemistry, and a consistent description of dust formation - still needs to be developed. In particular, the contribution of transparent grains to the acceleration of matter close

${ }^{\star}$ NOEMA data (FITS format) are only available at the CDS via anonymous ftp to cdsarc.u-strasbg.fr (130.79.128.5) or via http: //cdsarc.u-strasbg.fr/viz-bin/cat/J/A+A/658/A135 to the stellar photosphere (Norris et al. 2012) still needs to be assessed.

The mechanisms shaping circumstellar environments around asymptotic giant branch (AGB) stars are vividly debated. Among them, magnetic fields (Matt et al. 2000; Duthu et al. 2017), binarity (Theuns \& Jorissen 1993; Mastrodemos \& Morris 1999; Decin et al. 2020), stellar rotation (Dorfi \& Höfner 1996), and common-envelope evolution (Olofsson et al. 2015; Glanz \& Perets 2018) have been considered.

A major difficulty is to explain the observed velocity field in axi-symmetrical sources, with larger velocities at high latitudes than at low latitudes (Hoai et al. 2014; Nhung et al. 2015b). Also, recent observations of rotating structures and streams bring additional conundrums (Tuan-Anh et al. 2019; Hoai et al. 2019). 
We have concentrated our efforts on two relatively close $(d \sim 150 \mathrm{pc})$ sources that show composite profiles in CO rotational lines (Winters et al. 2003): EP Aqr (Winters et al. 2007, hereafter referred to as W2007), and RS Cnc (Libert et al. 2010). Data obtained at IRAM show that these two sources have an axi-symmetrical structure with a low-velocity $\left(\sim 2 \mathrm{~km} \mathrm{~s}^{-1}\right)$ wind close to the equatorial plane, and faster $\left(\sim 8 \mathrm{~km} \mathrm{~s}^{-1}\right)$ outflows around the polar axes (Hoai et al. 2014; Nhung et al. 2015b). For EP Aqr, W2007 find a radial dependence of the density showing intermediate maxima. Additional data obtained with ALMA (Nhung et al. 2019b; Homan et al. 2018b) reveal a spiral structure explaining the earlier W2007 results.

RS Cnc is one of the best examples of the interaction between the stellar wind from an AGB star and the surrounding interstellar medium (Hoai et al. 2014). Its high declination makes RS Cnc an ideal target for the Northern Extended Millimeter Array (NOEMA). Previous studies based on IRAM data show that it is a twin of EP Aqr, but observed at a different angle, with a polar axis inclined at about $30^{\circ}$ with respect to the line of sight (Libert et al. 2010; Hoai et al. 2014; Nhung et al. 2015b). This is favorable for studying polar and equatorial structures simultaneously, whereas the different viewing angle between EP Aqr and RS Cnc can be exploited to discriminate between different models in explaining the observed composite CO line profiles (Le Bertre et al. 2016). In contrast to EP Aqr, technetium is detected in the atmosphere of RS Cnc (Lebzelter \& Hron 1999), proving that it is evolving along the thermal pulsing asymptotic giant branch (TP-AGB) in the Hertzsprung-Russell (HR) diagram. From a chemical point of view, RS Cnc is in a slightly more advanced evolutionary stage on the AGB, as indicated by its spectral classification as an MS star (see below) and by a higher photospheric ratio of ${ }^{12} \mathrm{C} /{ }^{13} \mathrm{C}(\sim 35$; Smith \& Lambert (1986), but see Sect. 4.1 for an improved evaluation based on $\mathrm{CO}$ rotational lines from the circumstellar environment).

$\mathrm{RS}$ Cnc is a semi-regular variable star with periods of $\approx 122 \mathrm{~d}$ and $\approx 248$ days (Adelman \& Dennis 2005), located at a distance of $\approx 150$ pc (Gaia Collaboration 2021; Bailer-Jones et al. 2021). It is listed as S-star CSS 589 in Stephenson (1984) based on its spectral classification M6S given in Keenan (1954). With its weak $\mathrm{ZrO}$ bands, its chemical type is intermediate between $\mathrm{M}$ and $\mathrm{S}$ (Keenan 1954). The stellar temperature is estimated to $T_{*} \approx 3200 \mathrm{~K}$ and its luminosity is $L_{*} \approx 4950 L_{\odot}$ (Dumm \& Schild 1998). From CO rotational line observations, two circumstellar wind components were identified: an equatorial structure expanding at about $2 \mathrm{~km} \mathrm{~s}^{-1}$ and a bipolar outflow reaching a terminal velocity of $v_{\text {exp }} \approx 8 \mathrm{~km} \mathrm{~s}^{-1}$ (Libert et al. 2010; Hoai et al. 2014), carrying mass-loss rates of $4 \times 10^{-8} M_{\odot} \mathrm{yr}^{-1}$ and $8 \times 10^{-8} M_{\odot} \mathrm{yr}^{-1}$, respectively (see Sect. 4.1 for an improved value of the mass-loss rate derived here). Lines of ${ }^{12} \mathrm{CO}$, ${ }^{13} \mathrm{CO}, \mathrm{SiO}$, and $\mathrm{HI}$ were detected from previous observations at millimeter $(\mathrm{mm})$ and radio wavelengths (Nyman et al. 1992; Danilovich et al. 2015; de Vicente et al. 2016; Gérard \& Le Bertre 2003; Matthews \& Reid 2007).

NOEMA was recently equipped with the wide band correlator PolyFiX, covering a total bandwidth of $15.6 \mathrm{GHz}$ and therefore offering the potential to observe several lines from different species simultaneously. In this paper we present new data obtained with NOEMA in D- and A-configuration, complemented by short spacing observations obtained at the IRAM $30 \mathrm{~m}$ telescope. Observational details are summarized in Sect. 2 and our results are presented in Sect. 3. Section 4 contains a discussion of the morphological structures and compares them to similar structures found in EP Aqr. Our conclusions are summarized in Sect. 5.

\section{Observations}

New observations of RS Cnc have been obtained in $\mathrm{CO}(2-1)$ with NOEMA/WideX in the (extended) nine-antenna Aconfiguration in December 2016 (Nhung et al. 2018) and with NOEMA/PolyFiX in the (compact) nine-antenna Dconfiguration during the science verification phase of PolyFiX in December 2017 and in the ten-antenna A-configuration in February 2020. The WideX correlator covered an instantaneous bandwidth of $3.8 \mathrm{GHz}$ in two orthogonal polarizations with a channel spacing of $2 \mathrm{MHz}$. Additionally, up to eight high-spectral resolution units could be placed on spectral lines, providing channel spacings down to $39 \mathrm{kHz}$. WideX was decommissioned in September 2017 and replaced in December 2017 by the new correlator PolyFiX. This new correlator simultaneously covers $7.8 \mathrm{GHz}$ in two sidebands and for both polarizations, and provides a channel spacing of $2 \mathrm{MHz}$ throughout the $15.6 \mathrm{GHz}$ total bandwidth. In addition, up to 128 high-spectral-resolution "chunks" can be placed in the $15.6 \mathrm{GHz}$-wide frequency range covered by PolyFiX for both polarizations, each providing a fixed channel spacing of $62.5 \mathrm{kHz}$ over their $64 \mathrm{MHz}$ bandwidth.

RS Cnc was observed with two individual frequency setups covering a total frequency range of $\approx 32 \mathrm{GHz}$ in the $1.3 \mathrm{~mm}$ atmospheric window (see Fig. 1). We used the two quasars J0923+282 and $0923+392$ as phase and amplitude calibrators; these were observed every $\approx 20 \mathrm{~min}$. Pointing and focus of the telescopes was checked about every hour, and corrected when necessary. The bandpass was calibrated on the strong quasars 3C84 and 3C273, and the absolute flux scale was fixed on MWC349 and LkHa101, respectively. The accuracy of the absolute flux calibration at $1.3 \mathrm{~mm}$ is estimated to be better than $20 \%$.

In order to add the short spacing information filtered out by the interferometer, in May and July 2020 we observed at the IRAM $30 \mathrm{~m}$ telescope maps of $1^{\prime}$ by $1^{\prime}$ using the On-The-Fly (OTF) mode. This turned out to be necessary for the ${ }^{12} \mathrm{CO}(2-$ 1) and ${ }^{13} \mathrm{CO}(2-1)$ lines but was not needed for the $\mathrm{SiO}$ lines, whose emitting region was found to be smaller than $\sim 3^{\prime \prime}$. In the case of the ${ }^{12} \mathrm{CO}(2-1)$ and ${ }^{13} \mathrm{CO}(2-1)$ lines, the interferometer filters out large-scale structures that account for about two-thirds and three-quarters, respectively, of the total line flux, information that is recovered by adding the short spacing data from the OTF map. A comparison of the respective line profiles is shown in Fig. A.1.

The data were calibrated and imaged within the GILDAS ${ }^{1}$ suite of software packages using CLIC for the NOEMA data calibration and the $u v$ table creation, CLASS for calibrating the OTF maps, and the MAPPING package for merging and subsequent $u v$ fitting, imaging, and self-calibration of the combined data sets. Continuum data were extracted for each sideband of the two frequency setups individually by filtering out spectral lines, and then averaging over $400 \mathrm{MHz}$ bins to properly rescale the $u v$ coordinates to the mean frequency of each bin. Phase self-calibration was performed on the corresponding continuum data. The gain table containing the self-calibration solutions was then applied to the spectral line $u v$ tables using the SELFCAL procedures provided in MAPPING.

The resulting data sets were imaged applying either natural weighting, or, on the high-signal-to-noise $(\mathrm{S} / \mathrm{N})$ cubes, by applying robust weighting with a threshold of 0.1 to increase the spatial resolution by typically a factor 2 . The resulting dirty maps were then CLEANed using the Hogbom algorithm (Högbom 1974).

\footnotetext{
1 https://www.iram.fr/IRAMFR/GILDAS
} 
Table 1. Properties of the combined data sets for all detected lines.

\begin{tabular}{|c|c|c|c|c|c|c|c|c|c|}
\hline Line & $\begin{array}{l}\text { Frequency } \\
(\mathrm{GHz})\end{array}$ & $\begin{array}{r}E_{u} / k \\
(\mathrm{~K})\end{array}$ & $\begin{array}{r}\text { Peak flux } \\
\text { (Jy) }\end{array}$ & $\begin{array}{r}\text { FWHP } \\
(\operatorname{arcsec})\end{array}$ & $\begin{array}{r}\text { beam size } \\
\left(\operatorname{arcsec}^{2}\right)\end{array}$ & $\begin{array}{l}\text { PA } \\
(\operatorname{deg})\end{array}$ & $\begin{array}{c}1 \sigma \text { noise } \\
\left(\mathrm{mJy} \mathrm{beam}^{-1}\right)\end{array}$ & $\begin{array}{r}\text { vel.res } \\
\left(\mathrm{km} \mathrm{s}^{-1}\right)\end{array}$ & Comments ${ }^{(a)}$ \\
\hline${ }^{12} \mathrm{CO}(2-1)$ & 230.538000 & 16.6 & $53.971 \pm 10.841$ & $6.16 \pm 0.01$ & $0.48 \times 0.30$ & 36 & 2.88 & 0.5 & $\mathrm{~A}+\mathrm{D}+30 \mathrm{~m}, \mathrm{rw}$ \\
\hline${ }^{13} \mathrm{CO}(2-1)$ & 220.398684 & 15.9 & $4.693 \pm 0.948$ & $7.20 \pm 0.01$ & $0.50 \times 0.31$ & 35 & 2.79 & 0.5 & $\mathrm{~A}+\mathrm{D}+30 \mathrm{~m}, \mathrm{rw}$ \\
\hline $\mathrm{SiO}(v=0,5-4)$ & 217.104919 & 31.3 & $17.464 \pm 3.523$ & $1.71 \pm 0.01$ & $0.51 \times 0.32$ & 36 & 3.38 & 0.5 & $\mathrm{~A}+\mathrm{D}, \mathrm{rw}, \mathrm{sc}$ \\
\hline $\mathrm{SiO}(v=1,5-4)$ & 215.596018 & 1800.2 & $0.105 \pm 0.025$ & $0.19 \pm 0.02$ & $0.58 \times 0.43$ & 38 & 1.71 & 1.0 & A, nw, sc, Feb 2020: no maser \\
\hline $\mathrm{SiO}(v=1,5-4)$ & 215.596018 & 1800.2 & $0.105 \pm 0.025$ & $0.19 \pm 0.02$ & $2.10 \times 1.80$ & 0 & 2.71 & 0.5 & D, nw, sc, Dec 2017: maser \\
\hline $\mathrm{SiO}(v=2,5-4)$ & 214.088575 & 3552.1 & $0.013 \pm 0.005$ & $0.35 \pm 0.09$ & $1.00 \times 0.74$ & 35 & 1.03 & 3.0 & $\mathrm{~A}+\mathrm{D}, \mathrm{nw}$, double peak profile $(?)$ \\
\hline $\mathrm{SiO}(v=0,6-5)$ & 260.518009 & 43.8 & $23.906 \pm 4.817$ & $1.62 \pm 0.01$ & $0.43 \times 0.26$ & 32 & 3.39 & 0.5 & $\mathrm{~A}+\mathrm{D}, \mathrm{rw}, \mathrm{sc}$ \\
\hline $\mathrm{SiO}(v=1,6-5)$ & 258.707324 & 1812.7 & $0.168 \pm 0.038$ & $0.11 \pm 0.01$ & $0.60 \times 0.42$ & 26 & 1.96 & 1.0 & $\mathrm{~A}+\mathrm{D}, \mathrm{nw}, \mathrm{sc}$ \\
\hline${ }^{29} \mathrm{SiO}(v=0,5-4)$ & 214.385752 & 30.9 & $5.372 \pm 1.083$ & $1.19 \pm 0.01$ & $0.52 \times 0.32$ & 37 & 1.12 & 3.0 & $\mathrm{~A}+\mathrm{D}, \mathrm{rw}, \mathrm{sc}$ \\
\hline $\mathrm{Si}^{17} \mathrm{O}(v=0,6-5)$ & 250.744695 & 42.1 & $0.340 \pm 0.076$ & $0.88 \pm 0.04$ & $1.90 \times 1.50$ & 36 & $4.15^{(b)}$ & 3.0 & $\mathrm{D}, \mathrm{nw}, \mathrm{sc}$ tentative identification \\
\hline${ }^{29} \mathrm{Si}^{17} \mathrm{O}(v=0,6-5)$ & 247.481525 & 41.6 & $0.020 \pm 0.008$ & $0.73 \pm 0.44$ & $1.90 \times 1.50$ & 26 & 2.10 & 3.0 & $\mathrm{D}, \mathrm{nw}, \mathrm{sc}$, tentative detection \\
\hline $\mathrm{SO}(5(5)-4(4))$ & 215.220653 & 44.1 & $0.455 \pm 0.093$ & $0.79 \pm 0.01$ & $0.51 \times 0.32$ & 36 & 1.16 & 3.0 & $\mathrm{~A}+\mathrm{D}, \mathrm{rw}, \mathrm{sc}$ \\
\hline $\mathrm{SO}(6(5)-5(4))$ & 219.949442 & 35.0 & $0.634 \pm 0.130$ & $0.80 \pm 0.01$ & $0.50 \times 0.31$ & 36 & 1.17 & 3.0 & $\mathrm{~A}+\mathrm{D}, \mathrm{rw}, \mathrm{sc}$ \\
\hline $\operatorname{SO}(6(6)-5(5))$ & 258.255826 & 56.5 & $0.870 \pm 0.178$ & $0.74 \pm 0.01$ & $0.43 \times 0.27$ & 32 & 1.59 & 3.0 & $\mathrm{~A}+\mathrm{D}, \mathrm{rw}, \mathrm{sc}$ \\
\hline $\mathrm{SO}(7(6)-6(5))$ & 261.843721 & 47.6 & $1.168 \pm 0.238$ & $0.78 \pm 0.01$ & $0.43 \times 0.26$ & 32 & 1.38 & 3.0 & $\mathrm{~A}+\mathrm{D}, \mathrm{rw}, \mathrm{sc}$ \\
\hline${ }^{34} \mathrm{SO}(6(5)-5(4))$ & 215.839920 & 34.4 & $0.030 \pm 0.009$ & $0.92 \pm 0.11$ & $0.91 \times 0.80$ & 69 & 0.86 & 3.0 & $\mathrm{~A}+\mathrm{D}, \mathrm{nw}$ \\
\hline${ }^{34} \mathrm{SO}(5(6)-4(5))$ & 246.663470 & 49.9 & $0.026 \pm 0.009$ & $0.93 \pm 0.14$ & $0.69 \times 0.48$ & 26 & 0.99 & 3.0 & $\mathrm{~A}+\mathrm{D}, \mathrm{nw}$ \\
\hline $\mathrm{SO}_{2}(16(3,13)-16(2,14))$ & 214.689394 & 147.8 & $0.021 \pm 0.006$ & $0.50 \pm 0.06$ & $0.90 \times 0.68$ & 37 & 1.06 & 3.0 & $\mathrm{~A}+\mathrm{D}, \mathrm{nw}, \mathrm{sc}$ \\
\hline $\mathrm{SO}_{2}(22(2,20)-22(1,21))$ & 216.643304 & 248.4 & $0.023 \pm 0.007$ & $0.38 \pm 0.05$ & $0.89 \times 0.67$ & 36 & 1.11 & 3.0 & $\mathrm{~A}+\mathrm{D}, \mathrm{nw}, \mathrm{sc}$ \\
\hline $\mathrm{SO}_{2}(28(3,25)-28(2,26))$ & 234.187057 & 403.0 & $0.022 \pm 0.006$ & $0.19 \pm 0.05$ & $0.71 \times 0.56$ & 46 & 1.28 & 3.0 & $\mathrm{~A}+\mathrm{D}, \mathrm{nw}, \mathrm{sc}$ \\
\hline $\mathrm{SO}_{2}(14(0,14)-13(1,13))$ & 244.254218 & 93.9 & $0.043 \pm 0.011$ & $0.43 \pm 0.03$ & $0.69 \times 0.49$ & 27 & 1.00 & 3.0 & $\mathrm{~A}+\mathrm{D}, \mathrm{nw}, \mathrm{sc}$ \\
\hline $\mathrm{SO}_{2}(10(3,7)-10(2,8))$ & 245.563422 & 72.7 & $0.025 \pm 0.007$ & $0.36 \pm 0.04$ & $0.69 \times 0.49$ & 28 & 1.00 & 3.0 & $\mathrm{~A}+\mathrm{D}, \mathrm{nw}, \mathrm{sc}$ \\
\hline $\mathrm{SO}_{2}(15(2,14)-15(1,15))$ & 248.057402 & 119.3 & $0.015 \pm 0.005$ & $0.26 \pm 0.06$ & $0.69 \times 0.48$ & 27 & 1.07 & 3.0 & $\mathrm{~A}+\mathrm{D}, \mathrm{nw}, \mathrm{sc}$ \\
\hline $\mathrm{SO}_{2}(32(4,28)-32(3,29))$ & 258.388716 & 531.1 & $0.020 \pm 0.006$ & $0.21 \pm 0.04$ & $0.63 \times 0.45$ & 25 & 1.10 & 3.0 & $\mathrm{~A}+\mathrm{D}, \mathrm{nw}, \mathrm{sc}$ \\
\hline $\mathrm{SO}_{2}(9(3,7)-9(2,8))$ & 258.942199 & 63.5 & $0.026 \pm 0.008$ & $0.49 \pm 0.05$ & $0.64 \times 0.45$ & 26 & 1.08 & 3.0 & $\mathrm{~A}+\mathrm{D}, \mathrm{nw}, \mathrm{sc}$ \\
\hline $\mathrm{SO}_{2}(30(4,26)-30(3,27))$ & 259.599448 & 471.5 & $0.022 \pm 0.005$ & $0.12 \pm 0.03$ & $0.63 \times 0.45$ & 25 & 1.03 & 3.0 & $\mathrm{~A}+\mathrm{D}, \mathrm{nw}, \mathrm{sc}$ \\
\hline $\mathrm{SO}_{2}(30(3,27)-30(2,28))$ & 263.543953 & 459.0 & $0.019 \pm 0.006$ & $0.16 \pm 0.04$ & $0.61 \times 0.42$ & 26 & 1.25 & 3.0 & $\mathrm{~A}+\mathrm{D}, \mathrm{nw}, \mathrm{sc}$ \\
\hline $\mathrm{SO}_{2}(34(4,30)-34(3,31))$ & 265.481972 & 594.7 & $0.020 \pm 0.006$ & $0.19 \pm 0.04$ & $0.61 \times 0.42$ & 26 & 1.27 & 3.0 & $\mathrm{~A}+\mathrm{D}, \mathrm{nw}, \mathrm{sc}$ \\
\hline $\mathrm{H}_{2} \mathrm{O}\left(v_{2}=1,5(5,0)-6(4,3)\right)$ & $232.686700^{(c)}$ & 3462.0 & $0.029 \pm 0.007$ & unresolved & $0.71 \times 0.57$ & 47 & 1.17 & 3.0 & $\mathrm{~A}+\mathrm{D}, \mathrm{nw}, \mathrm{sc}, \mathrm{JPL}$ \\
\hline $\mathrm{H}_{2} \mathrm{O}\left(v_{2}=1,7(7,0)-8(6,3)\right)$ & $263.451357^{(d)}$ & 4474.7 & $0.021 \pm 0.005$ & unresolved & $0.61 \times 0.42$ & 26 & 1.17 & 3.0 & $\mathrm{~A}+\mathrm{D}, \mathrm{nw}, \mathrm{sc}, \mathrm{JPL}$ \\
\hline $\mathrm{HCN}(3-2)$ & 265.886434 & 25.5 & $1.116 \pm 0.234$ & $0.76 \pm 0.01$ & $0.42 \times 0.26$ & 32 & 4.80 & 0.5 & $\mathrm{~A}+\mathrm{D}, \mathrm{rw}, \mathrm{sc}$ \\
\hline $\mathrm{H}^{13} \mathrm{CN}(3-2)$ & 259.011798 & 24.9 & $0.041 \pm 0.011$ & $0.71 \pm 0.05$ & $0.64 \times 0.45$ & 26 & 0.95 & 3.0 & $\mathrm{~A}+\mathrm{D}, \mathrm{nw}, \mathrm{sc}$ \\
\hline $\mathrm{PN}(N=5-4, J=6-5)$ & 234.935694 & 33.8 & $0.028 \pm 0.009$ & $0.80 \pm 0.10$ & $0.70 \times 0.56$ & 47 & 1.00 & 3.0 & $\mathrm{~A}+\mathrm{D}, \mathrm{nw}, \mathrm{sc}$ \\
\hline
\end{tabular}

Notes. Line frequencies and upper level energies are from the CDMS (Müller et al. 2005), unless otherwise stated. The quoted flux uncertainties include the rms of the fits and the absolute flux calibration accuracy of $20 \%$, the uncertainties quoted for the source sizes refer to the rms errors of the Gaussian fits (see text). ${ }^{(a)}$ A: NOEMA A-configuration, D: NOEMA D-configuration, $30 \mathrm{~m}$ : short spacing data, rw: robust weighting, nw: natural weighting, sc: self-calibrated, JPL: Spectral line catalog by NASA/JPL (Pickett et al. 1998). ${ }^{(b)}$ Increased noise at band edge. ${ }^{(c)}$ Belov et al. (1987). ${ }^{(d)}$ Pearson et al. (1991).

The beam characteristics and sensitivities of the individual combined data sets from A- and D-configuration (and including the pseudo-visibilities from the OTF maps, where appropriate) are listed in Table 1 for all detected lines.

\section{Results}

The PolyFiX data, covering the frequency ranges $213-221 \mathrm{GHz}$ (setup1, LSB), 228-236 GHz (setup1, USB), 243-251 GHz (setup2, LSB), and 258-266 GHz (setup2, USB) with two setups (see Fig. 1), showed different lines of $\mathrm{CO}$ and $\mathrm{SiO}$, and, for the first time, many lines of species like $\mathrm{SO}, \mathrm{SO}_{2}, \mathrm{HCN}$, and $\mathrm{PN}$ and some of their isotopologs. Furthermore, the data confirmed the $\mathrm{H}_{2} \mathrm{O}$ line at $232.687 \mathrm{GHz}$ already detected serendipitously with
WideX in 2016, with a second $\mathrm{H}_{2} \mathrm{O}$ line at $263.451 \mathrm{GHz}$ seen for the first time in RS Cnc.

All lines covered by the same setup ( 1 or 2 , see Fig. 1 ) share the same phase-, amplitude-, and flux calibration. All 32 detected lines are listed in Table 1.

\subsection{Continuum}

Figure 2 shows the continuum map from A-configuration only, using robust weighting to increase the spatial resolution to $0.39^{\prime \prime} \times 0.22^{\prime \prime}$ at PA $28^{\circ}$. After self-calibration, $\mathrm{S} / \mathrm{N}=492$ is obtained. The continuum source is unresolved, a point source fit results in a flux at $\approx 247 \mathrm{GHz}$ of $23.65 \pm 4.7 \mathrm{mJy}$ (where the quoted error accounts for the accuracy of the absolute flux calibration of 20\%) and a source position at RA $=09: 10: 38.780$ and 

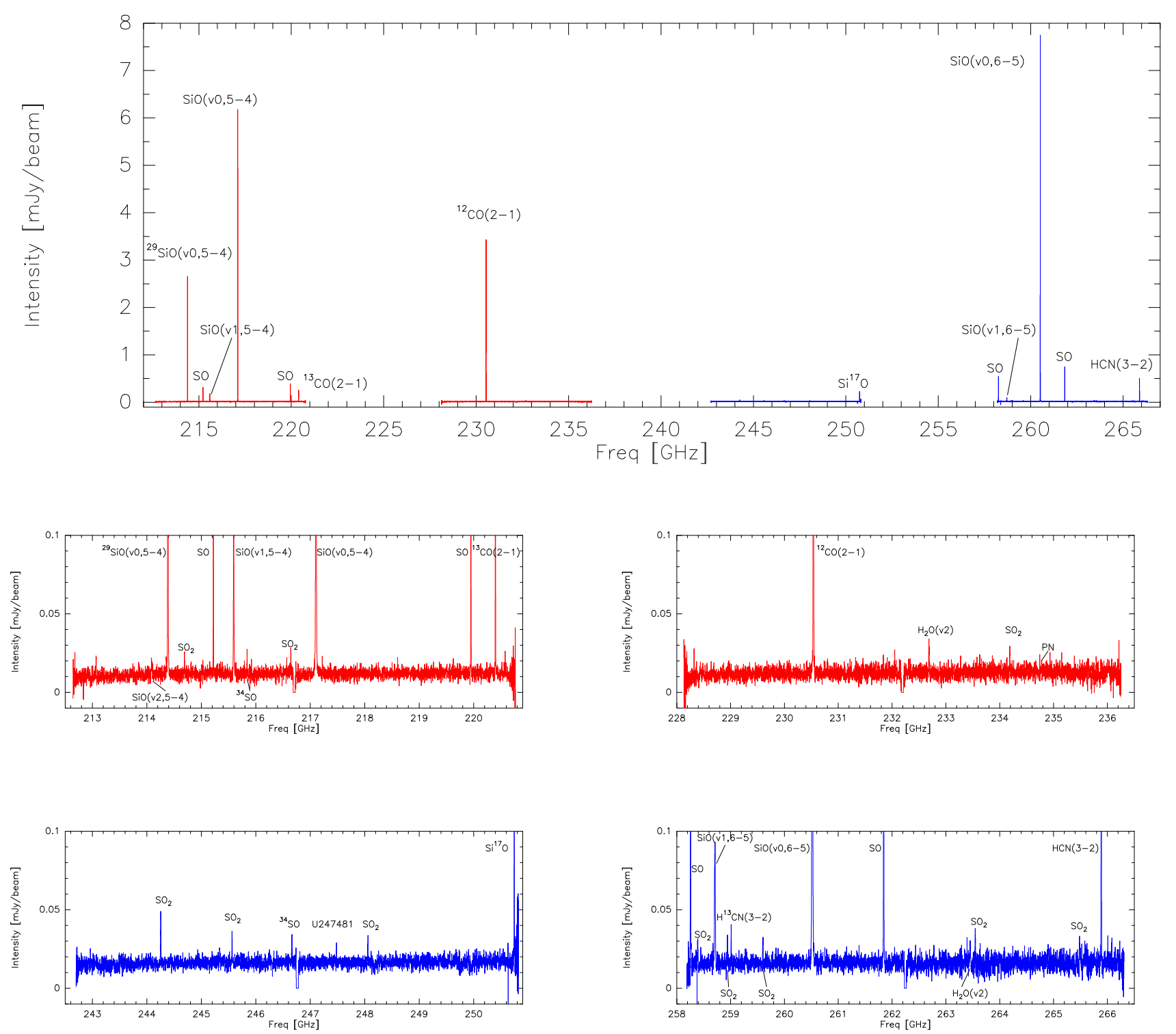

Fig. 1. Overview of the frequency ranges observed with PolyFiX using two spectral setups (setup1: red and setup2: blue, respectively). Lower diagrams: zoom onto the individual spectra covering $7.8 \mathrm{GHz}$ each. Upper row: setup1, lower row: setup2. The central $20 \mathrm{MHz}$ at the border between inner and outer baseband are blanked out, i.e., set to zero, as this region is contaminated by the LO2 separation of the $8 \mathrm{GHz}$-wide IF in the IF processor ("LO2 zone").

Dec $=30: 57: 46.62$ in February 2020. All line data cubes discussed in the remainder of this paper are re-centered on this continuum position.

The source position is offset from the J2000 coordinates by $-0.26^{\prime \prime}$ in RA and by $-0.68^{\prime \prime}$ in Dec, consistent with the proper

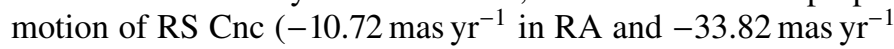
in Dec, Gaia Collaboration 2021; Bailer-Jones et al. 2021). From the PolyFiX data, spanning a total frequency range of about $53 \mathrm{GHz}$, we determine a spectral index of $1.99 \pm 0.09$ for RS Cnc in the $1 \mathrm{~mm}$ range, which is fully consistent with a black body spectrum of the continuum (see also Libert et al. 2010).

\subsection{Detected molecules and lines}

Within the total frequency coverage of about $32 \mathrm{GHz}$, we detect 32 lines of 13 molecules and isotopologs, including several transitions from vibrationally excited states. All these lines are listed in Table 1 and are presented in the following sections. The peak flux and FWHP of the line-emitting regions, as listed in Table 1, are determined by circular Gaussian fits in the $u v$-plane to the central channel (if the source is (partially) spatially resolved) or by point-source fits to the central channel (if the source is unresolved). All line profiles shown in the following sections in Fig. 3 and Figs. 5 through 14 are integrated over square apertures whose sizes are given in each figure caption. Two-component profiles are seen in $\mathrm{CO}$ and ${ }^{13} \mathrm{CO}$ only, and not in any other of the lines detected here.

We looked for but did not detect the vibrationally excited ${ }^{12} \mathrm{CO}(v=1,2-1)$ line, nor do we detect $\mathrm{C}^{18} \mathrm{O}(2-1)$, resulting in $3 \sigma$ upper limits for the line peaks of $6 \mathrm{mJy} \mathrm{beam}^{-1}$ and $3 \mathrm{mJy}_{\text {beam }}{ }^{-1}$, respectively (the ${ }^{12} \mathrm{CO}(v=1,2-1)$ line was not covered in our A-configuration data).

\subsection{1. $\mathrm{CO}$}

The profiles of ${ }^{12} \mathrm{CO}(2-1),{ }^{13} \mathrm{CO}(2-1)$ (see Fig. 3), and ${ }^{12} \mathrm{CO}(1-$ 0) (see Libert et al. 2010) show a very distinct shape composed of a broad component that extends out to $v_{\mathrm{lsr}, *} \pm 8 \mathrm{~km} \mathrm{~s}^{-1}$ and 


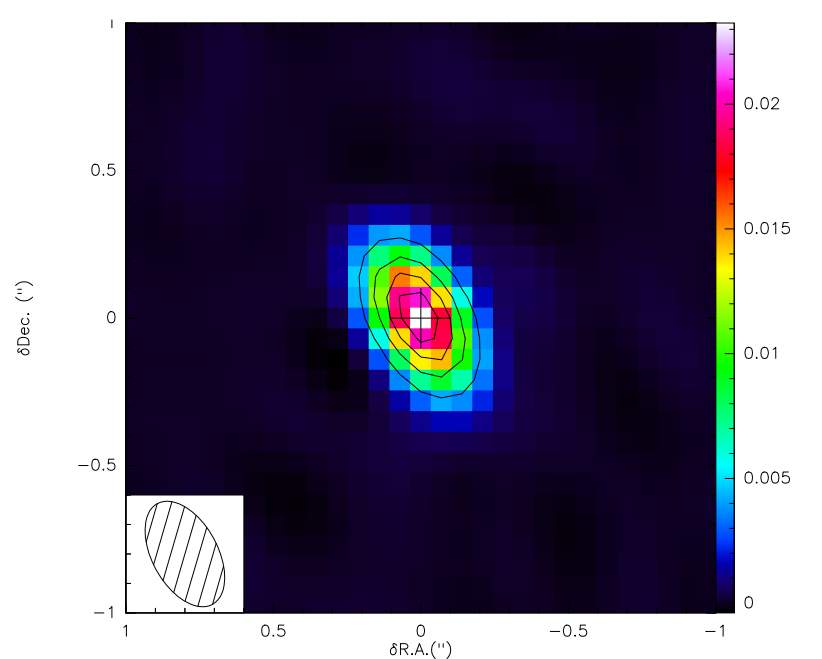

Fig. 2. Continuum map around $247 \mathrm{GHz}$ from A-configuration. Contours are plotted in $100 \sigma$ steps, where $1 \sigma$ is $47.6 \mu \mathrm{Jy}_{\text {beam }}{ }^{-1}$. The synthesized beam is indicated in the lower left corner.
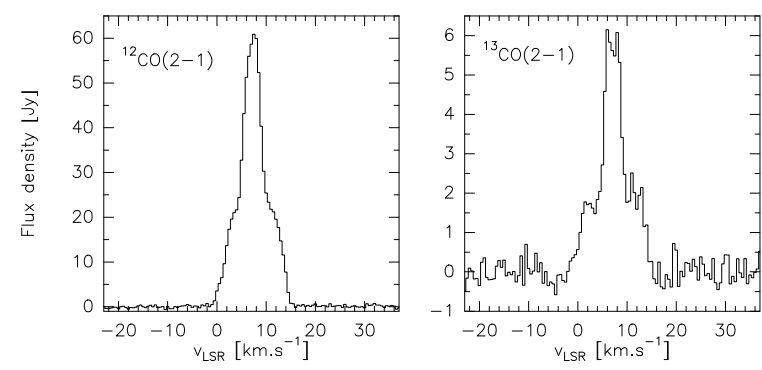

Fig. 3. CO line profiles, showing a two-component structure. Left: ${ }^{12} \mathrm{CO}(2-1)$. Right: ${ }^{13} \mathrm{CO}(2-1)$. A-configuration and D-configuration are merged, OTF data are added, and the spectral resolution is $0.5 \mathrm{~km} \mathrm{~s}^{-1}$. The CO emission is integrated over the central $22^{\prime \prime} \times 22^{\prime \prime}$, i.e., over the full field of view of the NOEMA antennas at $230 \mathrm{GHz}$.

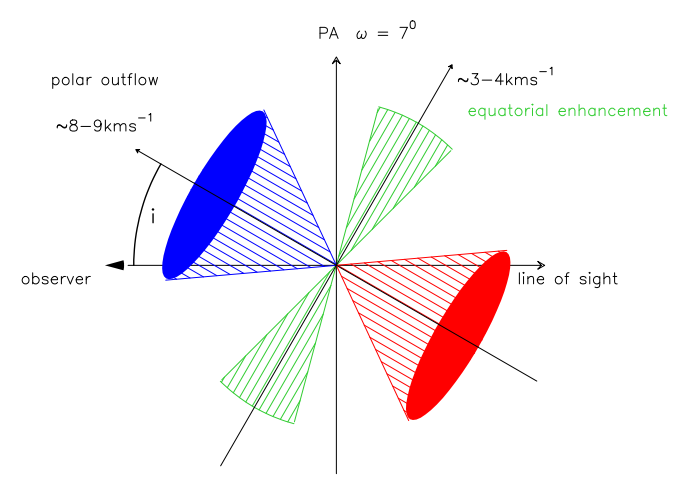

Fig. 4. Sketch of the geometrical structure of the wind components as inferred from the current data (see Sect. 4.1). The sketch is not to scale: there is a smooth transition between the equatorial enhancement and the polar outflows.

a narrow component indicating velocities of $\pm 2 \mathrm{~km} \mathrm{~s}^{-1}$ with respect to $v_{\mathrm{lsr}, *}=7 \mathrm{~km} \mathrm{~s}^{-1}$. Velocity-integrated intensity maps of $\mathrm{CO}$ are shown in Fig. 18, indicating a clear kinematic structure in the north-south direction. In Fig. 4, we present a schematic representation of the geometrical structure of RS Cnc as implied by the data; see Sect. 4.1. The CO emitting region is spatially extended, consisting of a dense equatorial structure that corresponds to the low-velocity expansion and an inclined, bipolar
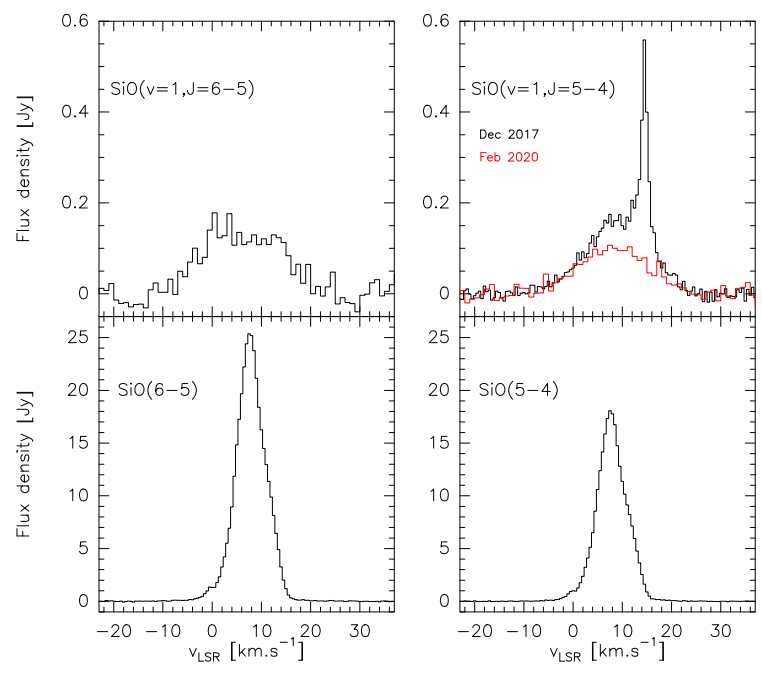

Fig. 5. Profiles of $\mathrm{SiO}$ ground-state and first vibrationally excited state lines. Left: $\mathrm{SiO}(6-5)$ : upper: $v=1$, lower: $v=0$. A-configuration and $\mathrm{D}$ configuration merged. Right: $\mathrm{SiO}(5-4)$ : upper: $v=1$, D-configuration (black) and A-configuration (red), lower: $v=0$, A-configuration and Dconfiguration merged. The spectral resolution is $1 \mathrm{~km} \mathrm{~s}^{-1}$ for $(v=1)$ and $0.5 \mathrm{~km} \mathrm{~s}^{-1}$ for the $(v=0)$ lines, respectively. The emission is integrated over the central $5^{\prime \prime} \times 5^{\prime \prime}$ aperture.

structure corresponding to an outflow at a projected velocity of $8 \mathrm{~km} \mathrm{~s}^{-1}$. These structures were discussed in Hoai et al. (2014) based on Plateau de Bure data obtained on ${ }^{12} \mathrm{CO}(2-1)$ and ${ }^{12} \mathrm{CO}(1-0)$ that had a spatial resolution of about $1^{\prime \prime}$. The model built by these latter authors was later refined by Nhung et al. (2018) based on ${ }^{12} \mathrm{CO}(2-1)$ data obtained with the WideX correlator in NOEMA's A-configuration, providing a spatial resolution of $0.44^{\prime \prime} \times 0.28^{\prime \prime}$. Nhung et al. (2018) find a position angle of the projected bipolar outflow axis of $\omega=7^{\circ}$ (measured counter-clockwise from north) and an inclination angle of the outflow axis with respect to the line of sight of $i=30^{\circ}$. The CO distribution is further investigated in Sect. 4.1 below.

Such a structure had already been found in the S-type star $\pi^{1}$ Gru (Sahai 1992), which was later confirmed by higher spatial resolution observations using ALMA (Doan et al. 2017). This object has a G0V companion (Feast 1953) and possibly a second, much closer companion (Homan et al. 2020). In Hoai et al. (2014), we reported for RS Cnc the possible presence of a companion seen in the ${ }^{12} \mathrm{CO}(1-0)$ channel maps at velocities around $6.6 \mathrm{~km} \mathrm{~s}^{-1}$ and located about $1^{\prime \prime}$ west-northwest of the continuum source. The new data allow for a more detailed study of this feature, which is presented in Sect. 4.1.

\subsection{2. $\mathrm{SiO}$}

We detect a suite of ${ }^{28} \mathrm{Si}^{16} \mathrm{O}$ (henceforth $\mathrm{SiO}$ ) transitions, including the vibrational ground-state lines of $\mathrm{SiO}(5-4)$ and $\mathrm{SiO}(6-5)$, the first and second vibrationally excited state of $\mathrm{SiO}(5-4)$, and the first vibrationally excited state of $\mathrm{SiO}(6-5)$. All $\mathrm{SiO}$ profiles are shown in Figs. 5 and 6. The spatial region emitting the vibrational ground-state lines extends out to about $2^{\prime \prime}$ from the continuum peak (see Table 1, Fig. 18, and Sect. 4.2). Interestingly, we detect a strong maser component on the $\operatorname{SiO}(v=1$, $J=5-4)$ line at $v_{\text {lsr }} \approx 14 \mathrm{~km} \mathrm{~s}^{-1}$ in the data obtained in December 2017, which had completely disappeared when we re-observed RS Cnc in February 2020 (see Fig. 5). Such behavior is well known for pulsating AGB stars, and lends support to 


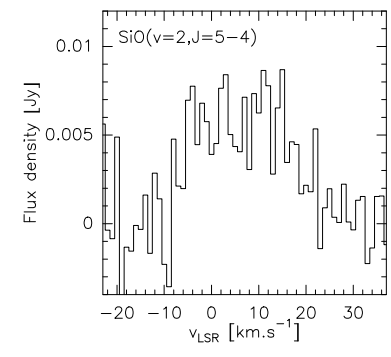

Fig. 6. Profile around the $\operatorname{SiO}(v=2, J=5-4)$ line frequency. Aconfiguration and D-configuration merged. The spectral resolution is $1 \mathrm{~km} \mathrm{~s}^{-1}$ and the emission is integrated over the central $1^{\prime \prime} \times 1^{\prime \prime}$ aperture.

the idea that the $\mathrm{SiO}$ masers are excited by infrared pumping as opposed to collisional pumping (see, e.g., Pardo et al. 2004).

The $\operatorname{SiO}(v=2, J=5-4)$ line is detected above the $3 \sigma$ level of $3 \mathrm{mJy}_{\text {beam }}{ }^{-1}$ over a broad range of Doppler velocities from at least -5 to $18 \mathrm{~km} \mathrm{~s}^{-1}$ (Fig. 6). Given its high excitation energy $(\sim 3500 \mathrm{~K})$, we expect this line to trace exclusively the innermost region around $\mathrm{RS} \mathrm{Cnc}$, as was the case in $o$ Cet, where $\mathrm{SiO}(v=2)$ absorption and emission was spatially resolved by ALMA (Wong et al. 2016). Its broad line width suggests that it may trace the same high-velocity wings seen in other detected $\mathrm{SiO}$ lines (Sect. 3.3). However, our detection is too weak to allow for a detailed study of the morpho-kinematics of the emission.

At the upper edge of the LSB of setup 2 at $250.744 \mathrm{GHz}$, we serendipitously detect a strong line that we identify as ground-state $\mathrm{Si}^{17} \mathrm{O}(6-5)$ at $250.7446954 \mathrm{GHz}$ (Müller et al. 2013) from the Cologne Database of Molecular Spectroscopy $\left(\mathrm{CDMS}^{2}\right.$, Müller et al. 2005); the profile is shown in Fig. 7. This line and other transitions of $\mathrm{Si}^{17} \mathrm{O}$ have already been detected in a number of well-studied objects, such as the S-type star W Aql (De Beck \& Olofsson 2020), the M-type star R Dor (De Beck \& Olofsson 2018), and the evolved, high-mass-lossrate oxygen-rich star IK Tau (Velilla Prieto et al. 2017). No other $\mathrm{Si}^{17} \mathrm{O}$ transitions are covered in our setups, but there is a highly excited $\mathrm{H}_{2} \mathrm{O}$ line at $250.7517934 \mathrm{GHz}\left(v_{2}=2, J\left(K_{a}, K_{c}\right)=\right.$ $\left.9(2,8)-8(3,5) ; E_{u} / k=6141 \mathrm{~K}\right)$ listed in the JPL catalog ${ }^{3}$ and predicted by Yu et al. (2012) from the Bending-Rotation approach analysis. If the detected line was $\mathrm{H}_{2} \mathrm{O}$ emission, it would be redshifted from the systemic velocity by about $9 \mathrm{~km} \mathrm{~s}^{-1}$. As indicated by the modeling of Gray et al. (2016), the $250.752 \mathrm{GHz}$ line may exhibit strong maser action in regions of hot gas $\left(T_{\text {kin }}=1500 \mathrm{~K}\right)$ with cool dust $\left(T_{\mathrm{d}} \leq 1000 \mathrm{~K}\right)$. While we cannot unequivocally exclude some contamination from a potential new, redshifted $\mathrm{H}_{2} \mathrm{O}$ maser, we consider $\mathrm{Si}^{17} \mathrm{O}$ a more likely identification of the $250.744 \mathrm{GHz}$ emission. From the respective integrated line intensities of $\mathrm{Si}^{16} \mathrm{O}(6-5)$ and $\mathrm{Si}^{17} \mathrm{O}(6-5)$, which are $\sim 163 \mathrm{Jy} \mathrm{km} \mathrm{s}^{-1}$ and $\sim 3 \mathrm{Jy} \mathrm{km} \mathrm{s}^{-1}$, and taking the difference of the Einstein coefficients of the transitions into account, we estimate the isotopolog ratio ${ }^{16} \mathrm{O} /{ }^{17} \mathrm{O} \sim 50$, assuming equal excitation conditions for both transitions and optically thin emission of both lines. This value is much lower than the solar isotopic ratio of $\sim 2700$ (Lodders et al. 2009) due to dredge-up events (Karakas \& Lattanzio 2014; Hinkle et al. 2016) and is broadly consistent with those obtained in the M-type star R Dor and the S-type star W Aql (61-74; De Beck \& Olofsson 2018, 2020). The initial mass of RS Cnc is about $1.5 M_{\odot}$ (Libert et al. 2010) ${ }^{4}$,

2 https://cdms.astro.uni-koeln.de

3 https://spec.jpl.nasa.gov/ftp/pub/catalog/catform. html

4 As quoted in Libert et al. (2010), the value of $1.5 M_{\odot}$ was estimated by Busso \& Palmerini (their priv. comm.) using the FRANEC
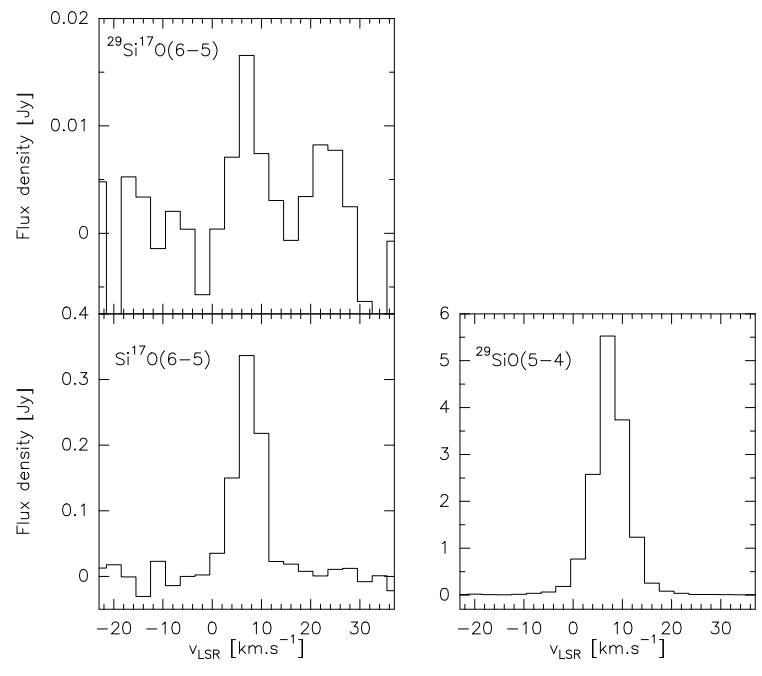

Fig. 7. Line profiles of $\mathrm{SiO}$ isotopologs. Upper left: profile of the 247.482 GHz line, possibly ${ }^{29} \mathrm{Si}^{17} \mathrm{O}(6-5)$; D-configuration, only. Lower left: $\mathrm{Si}^{17} \mathrm{O}(6-5)$ : D-configuration, only (line was not covered in Aconfiguration). Right: ${ }^{29} \mathrm{SiO}(5-4)$; A-configuration and D-configuration merged. The spectral resolution in all cases is $3 \mathrm{~km} \mathrm{~s}^{-1}$ and the emission is integrated over the central $5^{\prime \prime} \times 5^{\prime \prime}$ aperture.

which is in the same range as $\mathrm{R}$ Dor $\left(1.4 M_{\odot}\right.$; De Beck \& Olofsson 2018) and W Aql (1.6 $M_{\odot}$; De Nutte et al. 2017) that gives a ${ }^{16} \mathrm{O} /{ }^{17} \mathrm{O}$ ratio of $<1000$ (Hinkle et al. 2016). However, we note that the oxygen isotopic ratio $\left({ }^{16} \mathrm{O} /{ }^{17} \mathrm{O}\right)$ derived from the line intensity ratio is likely underestimated if the $\mathrm{Si}^{16} \mathrm{O}$ line is not optically thin, as has been shown in De Beck \& Olofsson (2018), who obtained a value of $\sim 400$ in $\mathrm{R}$ Dor with radiative transfer modeling. Indeed, we demonstrate in Sect. 4.2 that the $\mathrm{Si}^{16} \mathrm{O}$ emission in RS Cnc is optically thick, especially within a projected radius of $\sim 1^{\prime \prime}$. A photospheric ${ }^{16} \mathrm{O} /{ }^{17} \mathrm{O}$ ratio of $710 \mathrm{in}$ RS Cnc (=HR 3639) was estimated by Smith \& Lambert (1990) from the spectra of near-infrared overtone band transitions of $\mathrm{C}^{16} \mathrm{O}$ and $\mathrm{C}^{17} \mathrm{O}$, which is probably a more realistic ratio. We do not cover $\mathrm{C}^{17} \mathrm{O}(2-1)$ in our setups and therefore cannot give an independent estimate of the ${ }^{16} \mathrm{O} /{ }^{17} \mathrm{O}$ ratio. $\mathrm{As} \mathrm{Si}^{18} \mathrm{O}(6-5)$ and $\mathrm{C}^{18} \mathrm{O}(2-1)$ are either not covered or not detected, there is not enough information from our data to obtain a meaningful constraint on the initial stellar mass from oxygen isotopic ratios (e.g. from the ${ }^{17} \mathrm{O} /{ }^{18} \mathrm{O}$ ratio; De Nutte et al. 2017).

We detect a line at $247.482 \mathrm{GHz}$ at low $\mathrm{S} / \mathrm{N}$ that might be identified as ${ }^{29} \mathrm{Si}^{17} \mathrm{O}(v=0, J=6-5)$ at $247.4815250 \mathrm{GHz}$ based on the line list by Müller et al. (2013) and used in the CDMS (see Fig. 7). However, in contrast to $\mathrm{Si}^{17} \mathrm{O}(6-5),{ }^{29} \mathrm{Si}^{17} \mathrm{O}(6-5)$ has never been detected; only higher-J lines of ${ }^{29} \mathrm{Si}^{17} \mathrm{O}$ have been tentatively detected in R Dor $(J=7-6$ and $J=8-7$, De Beck \& Olofsson 2018). More specifically, the $247.482 \mathrm{GHz}$ line is seen with an integrated line intensity of $\sim 0.08 \mathrm{Jy} \mathrm{km} \mathrm{s}^{-1}$ in our D-configuration data only, observed in December 2017, but it does not show up in the A-configuration data, taken in February 2020. This may largely be due to the much reduced brightness

stellar evolution code (Cristallo et al. 2011) and the molecular abundances determined by Smith \& Lambert. Smith \& Lambert (1990) reported oxygen isotopic ratios of ${ }^{16} \mathrm{O} /{ }^{17} \mathrm{O}=710$ and ${ }^{16} \mathrm{O} /{ }^{18} \mathrm{O}=440$ in RS Cnc (their Table 9). The ${ }^{17} \mathrm{O} /{ }^{18} \mathrm{O}$ ratio of 0.62 corresponds to an initial mass of 1.4-1.5 $M_{\odot}$ in the comparative study of De Nutte et al. (2017), who investigated the ${ }^{17} \mathrm{O} /{ }^{18} \mathrm{O}$ isotopic ratio as a sensitive function of initial mass of low-mass stars based on the models of Stancliffe et al. (2004), Karakas \& Lattanzio (2014), and the FRANEC model. 

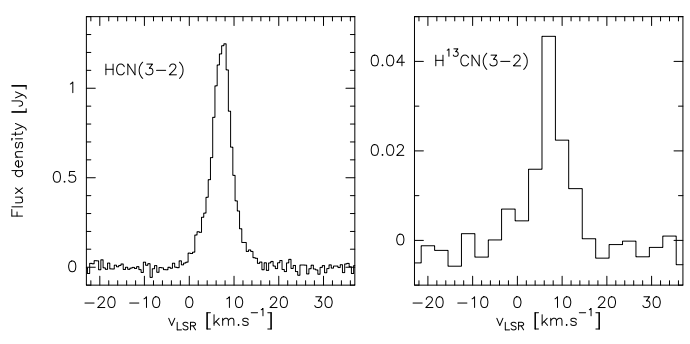

Fig. 8. HCN line profiles. Left: $\mathrm{HCN}(3-2)$; A-configuration and Dconfiguration merged with a spectral resolution of $0.5 \mathrm{~km} \mathrm{~s}^{-1}$. Right: $\mathrm{H}^{13} \mathrm{CN}(3-2)$; A-configuration and D-configuration merged with a spectral resolution of $3 \mathrm{~km} \mathrm{~s}^{-1}$. The emission of both lines is integrated over the central $2^{\prime \prime} \times 2^{\prime \prime}$.

sensitivity in the A-configuration, which is a factor of approximately 15 smaller because of the smaller synthesized beam area rather than some variable maser action in this line. Based on the D-configuration data, the source position of the $247.482 \mathrm{GHz}$ emission appears slightly offset toward the northwest direction from the $\mathrm{Si}^{17} \mathrm{O}(6-5)$ emission. Further data on ${ }^{29} \mathrm{Si}^{17} \mathrm{O}$, possibly covering the $J=6-5, J=7-6$, and $J=8-7$ transitions, would be needed to draw any firm conclusion.

\subsection{3. $\mathrm{HCN}$}

We clearly detect the $\mathrm{HCN}(3-2)$ and $\mathrm{H}^{13} \mathrm{CN}(3-2)$ lines; the profiles are displayed in Fig. 8, and velocity-integrated intensity maps of both species are shown in Fig. B.1. Both lines are slightly spatially resolved and a circular Gaussian fit to $\mathrm{HCN}(3-2)$ gives a peak flux of $1.12 \mathrm{Jy}$ and a FWHP size of $0.76^{\prime \prime}$ on the merged data. To our knowledge, this is the first detection of $\mathrm{HCN}$ and $\mathrm{H}^{13} \mathrm{CN}$ in RS Cnc (see Sect. 4.4). From the firstmoment map (shown in Fig. 17, left), a clear velocity pattern is evident that indicates possible rotation in the HCN-emitting region (see Sect. 3.4). Also, the velocity-integrated intensity maps presented in Fig. B.1 show a clear kinematic structure in the east-west direction.

Formation of the HCN molecule in oxygen-rich environments is further discussed in Sect. 4.4. A modeling using the 1D local thermodynamic equilibrium (LTE) radiative transfer code XCLASS (Möller et al. 2017, see Appendix D) gives a column density for $\mathrm{HCN}$ in RS Cnc of $\mathrm{N}_{\mathrm{HCN}}=1.6 \times 10^{15} \mathrm{~cm}^{-2}$, corresponding to an abundance of $X\left(\mathrm{HCN} / \mathrm{H}_{2}\right)=6.6 \times 10^{-7}$. This value is well within the range found for other M- and S-type stars as modeled by Schöier et al. (2013), who find $X\left(\mathrm{HCN} / \mathrm{H}_{2}\right)$ equal to a few times $10^{-7}$ (for more details see Sect. 4.4 and Appendix D).

\subsection{4. $\mathrm{H}_{2} \mathrm{O}$}

The WideX spectrum obtained in A-configuration in December 2016 serendipitously revealed a line at $232.687 \mathrm{GHz}$ that we ascribe to the $J\left(K_{a}, K_{c}\right)=5(5,0)-6(4,3)$ transition of $\mathrm{o}-\mathrm{H}_{2} \mathrm{O}$ in the $v_{2}=1$ vibrational state. The $\mathrm{H}_{2} \mathrm{O}$ source is weak and seems still unresolved within the synthesized beam of $0.5^{\prime \prime} \times 0.34^{\prime \prime}$ obtained in the A-configuration in February 2020, consistent with its high upper-state energy of $3462 \mathrm{~K}$. The line profile is shown in Fig. 9. With the follow-up observations employing PolyFiX in D-configuration and A-configuration we also covered and detected the $263.451 \mathrm{GHz}$ o- $\mathrm{H}_{2} \mathrm{O} v_{2}=1, J\left(K_{a}, K_{c}\right)=$ $7(7,0)-8(6,3)$ line (Fig. 9, right; $\left.E_{u} / k=4475 \mathrm{~K}\right)$. Both lines are resampled to a resolution of $3 \mathrm{~km} \mathrm{~s}^{-1}$, data are merged from A-configuration and D-configuration, and the emission is
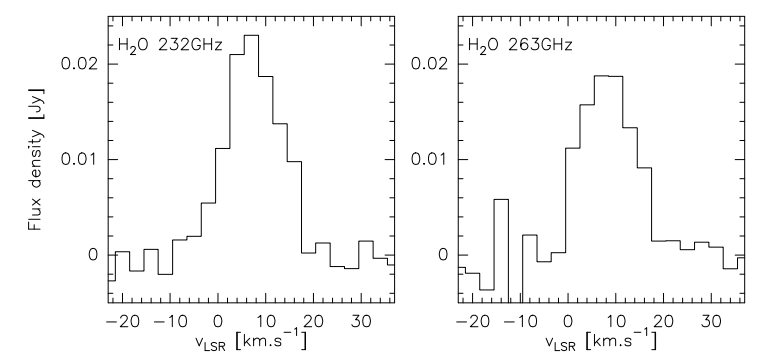

Fig. 9. $\mathrm{H}_{2} \mathrm{O}$ line profiles. Left: $\mathrm{H}_{2} \mathrm{O}$ line at $232.687 \mathrm{GHz}$. Right: $\mathrm{H}_{2} \mathrm{O}$ line at $263.451 \mathrm{GHz}$. Data are merged from A-configuration and Dconfiguration, the spectral resolution is $3 \mathrm{~km} \mathrm{~s}^{-1}$, and the emission of both lines is integrated over the central $1^{\prime \prime} \times 1^{\prime \prime}$ aperture.

integrated over an aperture of $1^{\prime \prime} \times 1^{\prime \prime}$. Intensity maps of both lines are shown in Fig. B.2, testifying to the compactness of the $\mathrm{H}_{2} \mathrm{O}$-emitting region.

These are the first detections of millimeter vibrationally excited $\mathrm{H}_{2} \mathrm{O}$ emission in $\mathrm{RS}$ Cnc. We note that the $22 \mathrm{GHz}$ $\mathrm{H}_{2} \mathrm{O}$ maser in the ground state was tentatively detected by Szymczak \& Engels (1995) in one of the two epochs they covered, but the $22 \mathrm{GHz}$ line is not detected in other observations (Dickinson et al. 1973; Lewis 1997; Han et al. 1995; Yoon et al. 2014). RS Cnc also shows clear photospheric $\mathrm{H}_{2} \mathrm{O}$ absorption at $2.7 \mu \mathrm{m}$ (Merrill \& Stein 1976; Noguchi \& Kobayashi 1993), and at $1.3 \mu \mathrm{m}\left(7500 \mathrm{~cm}^{-1}\right.$; Joyce et al. 1998), although the $\mathrm{H}_{2} \mathrm{O}$ band near $900 \mathrm{~nm}$ is not detected (Spinrad et al. 1966).

Both the 232 and $263 \mathrm{GHz}$ water lines have upper levels belonging to the so-called transposed backbone in the $v_{2}=1$ vibrationally excited state of $\mathrm{H}_{2} \mathrm{O}$, that is $K_{a}=J$ and $K_{c}=0$ or 1 (see Fig. 1 of Alcolea \& Menten 1993). The $232 \mathrm{GHz}$ line was first detected in evolved stars together with the $96 \mathrm{GHz}$ line from another transposed backbone upper level by Menten \& Melnick (1989) toward the red supergiant VY CMa and the AGB star W Hya. The latter is an M-type star with a similar mass-loss rate to RS Cnc. The authors find that the $232 \mathrm{GHz}$ line emission in both stars may be of (quasi-)thermal nature while the $96 \mathrm{GHz}$ line clearly showed maser action. The (unpublished) detection of the $263 \mathrm{GHz}$ line was mentioned in Alcolea \& Menten (1993), who also described a mechanism that may lead to a systematic overpopulation of the transposed backbone upper levels in the $v_{2}=1$ state of $\mathrm{H}_{2} \mathrm{O}$ in the inner region of circumstellar envelopes. If the vibrational decay routes (to the ground state) of the transposed backbone upper levels become more optically thick than the lower levels in the $v_{2}=1$ state, then differential radiative trapping may cause population inversion of these lines. Additional vibrationally excited $\mathrm{H}_{2} \mathrm{O}$ emission lines from transposed backbone upper levels were predicted and later detected in VY CMa by Menten et al. (2006) and Kamiński et al. (2013). We observed the $232 \mathrm{GHz}$ line in RS Cnc at three epochs (December 2016, December 2017, and February 2020) and the $263 \mathrm{GHz}$ line at the latter two epochs, and the emission appears to be stable in time for both lines. The profiles appear to be very similar, both are broad, even broader than the (ground-state) lines of other species reported here, and there is no sign for any narrow component in either of the two profiles at any of the epochs. As the lines should arise from a region very close to the star - compatible with their broad widths; see Sect. 3.3 - one might expect to see time variations due to the varying density and radiation field caused by the stellar pulsation, in particular if the emission were caused by maser action, as seen on the $\mathrm{SiO}(v=1,5-4)$ line observed in December 2017 (see Fig. 5). Also, the modeling of Gray et al. (2016) shows only very little inversion of the 

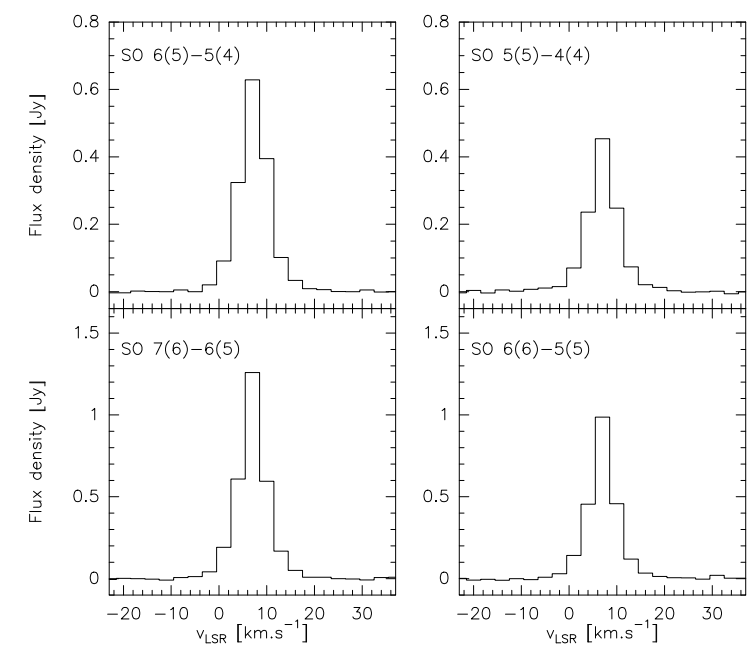

Fig. 10. Profiles of the four detected SO lines, with A-configuration and D-configuration merged. The spectral resolution is $3 \mathrm{~km} \mathrm{~s}^{-1}$ and the emission is integrated over the central $2^{\prime \prime} \times 2^{\prime \prime}$ aperture.
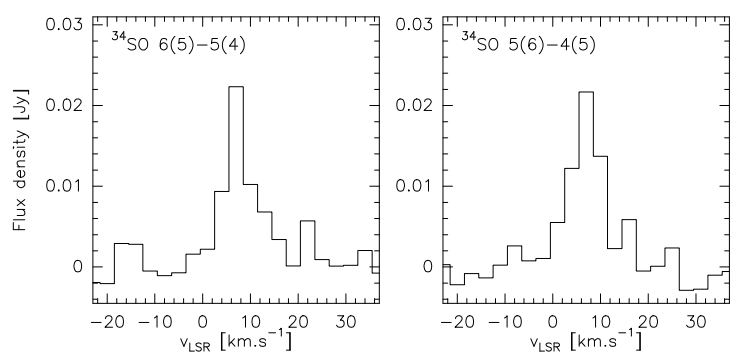

Fig. 11. Profiles of the two ${ }^{34} \mathrm{SO}$ lines detected here, with Aconfiguration and D-configuration merged. The spectral resolution is $3 \mathrm{~km} \mathrm{~s}^{-1}$ and the emission is integrated over the central $2^{\prime \prime} \times 2^{\prime \prime}$ aperture.

involved level populations for the $263 \mathrm{GHz} \mathrm{H}_{2} \mathrm{O}$ transition. We therefore think that both lines could be thermally excited. A definite assessment of the nature of the vibrationally excited $\mathrm{H}_{2} \mathrm{O}$ emission would however require some detailed modeling of the emission, together with high-sensitivity monitoring of the line profiles with high spectral resolution, possibly including other $\mathrm{H}_{2} \mathrm{O}$ lines from transposed backbone upper levels and/or known maser lines for comparison, which is beyond the scope of the present paper.

\subsubsection{SO}

Four lines of SO are detected (see Fig. 10) along with two lines of the isotopolog ${ }^{34} \mathrm{SO}$ (Fig. 11). These represent the first detections of SO and ${ }^{34}$ SO in RS Cnc. SO has been observed in several Mtype stars, including R Dor and W Hya, (Danilovich et al. 2016)), but remains undetected in S-type stars (e.g., W Aql, Decin et al. 2008; De Beck \& Olofsson 2020). All SO lines detected here are slightly spatially resolved with a FWHP around $0.8^{\prime \prime}$ and therefore seem to be emitted from the same region as HCN. Velocity-integrated intensity maps of SO are shown in Fig. B.3. The SO lines show the same velocity pattern (indicating rotation) as HCN, although the velocity resolution of the SO lines is only $3 \mathrm{~km} \mathrm{~s}^{-1}$; see Fig. B.3 and the first-moment map in the right panel of Fig. 17.

Using the integrated line strengths of $\mathrm{SO}(6(5)-5(4))$ and ${ }^{34} \mathrm{SO}(6(5)-5(4))$ found here $\left(\sim 4.69 \mathrm{Jy} \mathrm{km} \mathrm{s}^{-1}\right.$ and $\sim 0.20 \mathrm{Jy} \mathrm{km} \mathrm{s}^{-1}$, respectively) and taking the difference of the Einstein coefficients of the transitions into account, we estimate the isotopolog ratio ${ }^{32} \mathrm{SO} /{ }^{34} \mathrm{SO} \sim 23$, assuming equal excitation conditions for both transitions and optically thin emission of both lines. This value is in good agreement with the values of $21.6 \pm 8.5$ and $18.5 \pm 5.8$ derived from the radiative transfer models for M-type stars by Danilovich et al. (2016, 2020), respectively. We note that, for the S-type star W Aql, an $\mathrm{Si}^{32} \mathrm{~S} / \mathrm{Si}^{34} \mathrm{~S}$ isotopolog ratio of $10.6 \pm 2.6$ was derived by De Beck \& Olofsson (2020). As ${ }^{32} \mathrm{~S}$ is mainly produced by oxygen burning in massive stars and, to a lesser extent, in type Ia supernovae, and as ${ }^{34} \mathrm{~S}$ is formed by subsequent neutron capture (e.g., Nomoto et al. 1984; Wilson \& Matteucci 1992; Timmes et al. 1995; Hughes et al. 2008), the ${ }^{32} \mathrm{~S} /{ }^{34} \mathrm{~S}$ isotopic ratio remains virtually unaltered during AGB evolution (see, e.g. tables in the FRUITY ${ }^{5}$ database, Cristallo et al. 2011) and therefore should reflect the chemical initial conditions of the natal cloud from which the star has formed. The spread in the isotopic ratio seen among the different AGB stars mentioned above would then rather be indicative of the Galactic environment in which the star has formed (see, e.g., Chin et al. 1996; Humire et al. 2020) instead of reflecting any evolutionary effect. For the low-mass-loss-rate M-type stars R Dor and W Hya, Danilovich et al. (2016) reproduce their observed line profiles best with centrally peaked $\mathrm{SO}$ (and $\mathrm{SO}_{2}$ ) distributions, consistent with the maps presented in Fig. B.3.

\subsection{6. $\mathrm{SO}_{2}$}

In $\mathrm{SO}_{2}, 11$ lines are detected; their parameters are summarized in Table 2, and all profiles are shown in Fig. C.1. These are the first detections of $\mathrm{SO}_{2}$ in $\mathrm{RS}$ Cnc. A previous survey with the IRAM $30 \mathrm{~m}$ telescope by Omont et al. (1993) did not detect $\mathrm{SO}_{2}$ in $\mathrm{RS} \mathrm{Cnc}$ with an rms noise of $0.052 \mathrm{~K}$ (or $\sim 0.25 \mathrm{Jy}$ at $160.8 \mathrm{GHz})$. As an example, we show the $\mathrm{SO}_{2}(14(0,14)$ $13(1,13)$ ) line at $244.3 \mathrm{GHz}$, only in Fig. 12. A first-moment map of the $\mathrm{SO}_{2}(14(0,14)-13(1,13))$ line is shown in Fig. 17 in the middle left panel. Although the source remains barely resolved (source size $\left.\sim 0.43^{\prime \prime}\right)$ by the beam $\left(0.69^{\prime \prime} \times 0.49^{\prime \prime}\right)$, there is a signature of a rotating structure in $\mathrm{SO}_{2}$, as was also seen in EP Aqr (Homan et al. 2018b; Tuan-Anh et al. 2019). Integrated intensity maps of three $\mathrm{SO}_{2}$ lines $\left(\mathrm{SO}_{2}(9(3,7)-9(2,8))\right.$, which has the lowest upper level energy of the $\mathrm{SO}_{2}$ lines detected here $\left(E_{u}=64 \mathrm{~K}\right) ; \mathrm{SO}_{2}(14(0,14)-13(1,13))$, the strongest line, and $\mathrm{SO}_{2}(34(4,30)-34(3,31))$, which has the highest upper level energy of the detected lines, $E_{u}=595 \mathrm{~K}$ ) are shown in Fig. B.4. All lines show kinematic structure in the E-W direction, approximately orthogonal to the outflow structure seen in $\mathrm{CO}$ and $\mathrm{SiO}$, cf. Fig. 18.

We derive the rotational temperature and column density of the $\mathrm{SO}_{2}$-emitting region with a population diagram analysis (Sect. 3.6) and by an XCLASS modeling (Appendix D). Both methods give a similar rotational temperature of $\sim 320-350 \mathrm{~K}$ and a column density of $\sim 3.5 \times 10^{15} \mathrm{~cm}^{-2}$.

\subsubsection{PN}

We detect a line at $234.936 \mathrm{GHz}$ that we ascribe to the PN molecule, which would be the first detection of PN in RS Cnc. PN has been detected in several M-type stars (e.g., De Beck et al. 2013; Ziurys et al. 2018), and in the C-rich envelopes of IRC +10216 and CRL 2688 (Guélin et al. 2000; Cernicharo et al. 2000; Milam et al. 2008). The presence of PN in an MS-type star therefore does not seem to come as a surprise. However, RS Cnc

\footnotetext{
5 http://fruity.oa-teramo.inaf.it/
} 
Table 2. Parameters of the detected $\mathrm{SO}_{2}$ lines used for the population diagram analysis.

\begin{tabular}{rrrrrc}
\hline \hline $\begin{array}{r}\text { Frequency } \\
(\mathrm{GHz})\end{array}$ & $\begin{array}{r}W_{I}=\int S(v) \mathrm{d} v \\
\left(\mathrm{Jy} \mathrm{km} \mathrm{s}^{-1}\right)\end{array}$ & $g_{u}$ & $\begin{array}{r}\log _{10}\left(A_{\mathrm{ul}}\right) \\
\left(\mathrm{s}^{-1}\right)\end{array}$ & $\begin{array}{r}E_{u} / k \\
(\mathrm{~K})\end{array}$ & $\begin{array}{r}\theta_{a} \times \theta_{b} \\
\left(\mathrm{arcsec}^{2}\right)\end{array}$ \\
\hline 214.6894 & $0.141 \pm 0.0385$ & 33 & -4.0043 & 147.843 & $0.90 \times 0.68$ \\
216.6433 & $0.166 \pm 0.0434$ & 45 & -4.0329 & 248.442 & $0.89 \times 0.67$ \\
234.1871 & $0.160 \pm 0.0439$ & 57 & -3.8401 & 403.033 & $0.71 \times 0.56$ \\
244.2542 & $0.293 \pm 0.0698$ & 29 & -3.7855 & 93.901 & $0.69 \times 0.49$ \\
245.5634 & $0.170 \pm 0.0451$ & 21 & -3.9240 & 72.713 & $0.69 \times 0.49$ \\
248.0574 & $0.119 \pm 0.0333$ & 31 & -4.0939 & 119.328 & $0.69 \times 0.48$ \\
258.3887 & $0.153 \pm 0.0396$ & 65 & -3.6773 & 531.100 & $0.63 \times 0.45$ \\
258.9422 & $0.192 \pm 0.0524$ & 19 & -3.8800 & 63.472 & $0.64 \times 0.45$ \\
259.5994 & $0.182 \pm 0.0448$ & 61 & -3.6835 & 471.496 & $0.63 \times 0.45$ \\
263.5440 & $0.152 \pm 0.0448$ & 61 & -3.7227 & 459.038 & $0.61 \times 0.42$ \\
265.4820 & $0.168 \pm 0.0448$ & 69 & -3.6426 & 594.661 & $0.61 \times 0.42$ \\
\hline
\end{tabular}

Notes. Data are merged from A-configuration and D-configuration. Quoted errors include the rms errors of the Gaussian fits in the $u v$ plane and the absolute flux calibration accuracy of $20 \%$. The $\mathrm{SO}_{2}$ line parameters are retrieved from the CDMS and are based on the calculations by Lovas (1985) and Müller \& Brünken (2005).

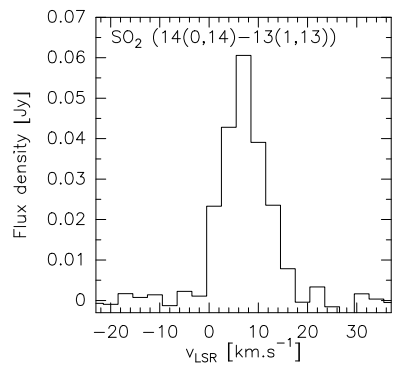

Fig. 12. Profile of $\mathrm{SO}_{2}(14(0,14)-13(1,13))$ with A-configuration and Dconfiguration merged, a spectral resolution of $3 \mathrm{~km} \mathrm{~s}^{-1}$, and emission integrated over the central $2^{\prime \prime} \times 2^{\prime \prime}$ aperture.

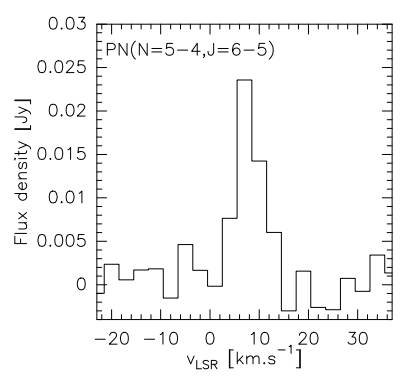

Fig. 13. Profile of $\mathrm{PN}(N=5-4, J=6-5)$ with A-configuration and Dconfiguration merged, a spectral resolution of $3 \mathrm{~km} \mathrm{~s}^{-1}$, and emission integrated over the central $2^{\prime \prime} \times 2^{\prime \prime}$ aperture.

appears to be the source with lowest mass-loss rate in which this molecule has been reported so far. The PN line profile is shown in Fig. 13. The line is spatially resolved at $0.8^{\prime \prime}$, which places it in about the same region as HCN and SO. The first-moment map of this line also shows signatures of rotation but due to the weakness of the line, the evidence is low. An integrated intensity map of PN is presented in Fig. B.5, showing that the line-emitting region is slightly spatially resolved. The $3 \sigma$ feature seen about $1.5^{\prime \prime}$ south of the phase center should not be considered as a detection but rather as a noise peak, as long as this structure is not confirmed by higher sensitivity observations.

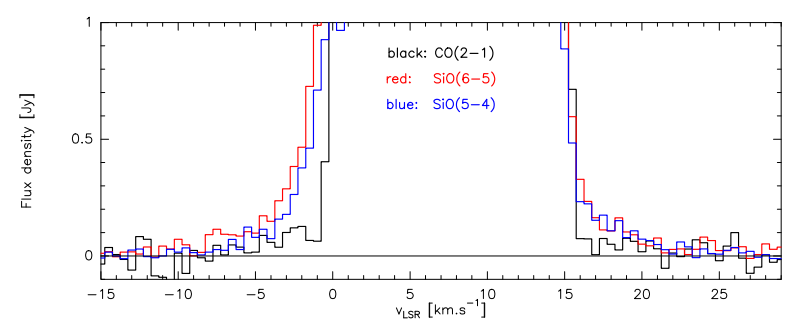

Fig. 14. Line wings in $\mathrm{SiO}(5-4)$ and $\mathrm{SiO}(6-5)$ compared to $\mathrm{CO}(2-1)$. The emission is integrated over the central $5^{\prime \prime} \times 5^{\prime \prime}$ aperture.
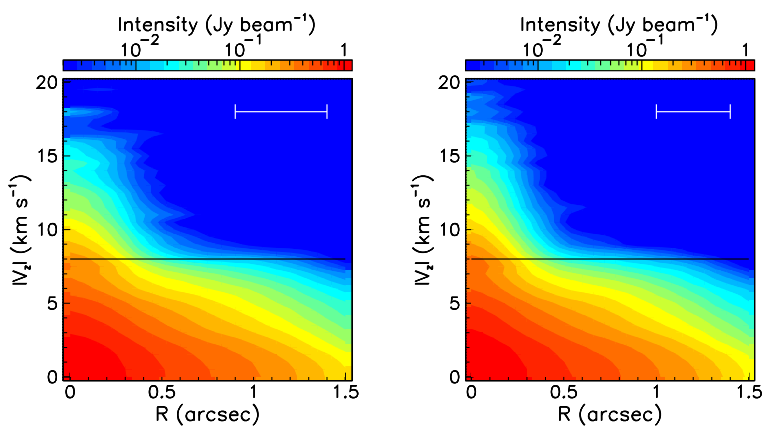

Fig. 15. High velocities close to the line of sight as seen in $\mathrm{SiO}$. PV maps are shown in the $V_{\mathrm{z}}$ vs. $R$ plane for $\mathrm{SiO}(5-4)$ (left) and $\mathrm{SiO}(6-5)$ (right). The horizontal black line indicates the wind terminal velocity as traced in $\mathrm{CO}$ and the white scale bar indicates the spatial resolution. $R=\sqrt{(\delta \mathrm{Dec})^{2}+(\delta \mathrm{RA})^{2}},\left|V_{\mathrm{z}}\right|=\left|v_{\mathrm{lsr}}-v_{\mathrm{lsr}, *}\right|$.

\subsection{High-velocity wings in $\mathrm{SiO}$, and in other molecules}

In $\mathrm{SiO}$, five lines in three different vibrational states $(v=0,1,2)$ are detected (see Figs. 5 and 6). The vibrational ground-state lines clearly indicate the presence of material at velocities much higher than the wind terminal velocity of $\sim 8 \mathrm{~km} \mathrm{~s}^{-1}$ as traced by CO lines at this stellar latitude (see Sect. 4.1). This is illustrated in Fig. 14, and in Fig. 15 where we define $v_{\mathrm{z}}=v_{\mathrm{lsr}}-v_{\mathrm{lsr}, *}$, the Doppler velocity relative to the star. The high-velocity region is centered on the line of sight and is confined to the inner $\approx 0.3^{\prime \prime}$; see Fig. 15 . A similar feature was seen in high-spatialresolution observations of other oxygen-rich, low-mass-loss-rate 
AGB stars, such as W Hya (Vlemmings et al. 2017), EP Aqr (Tuan-Anh et al. 2019), $o$ Cet (Hoai et al. 2020), R Dor (Decin et al. 2018; Nhung et al. 2019a, 2021), and in 15 out of 17 sources observed in the ALMA Large Program ATOMIUM (Decin et al. 2020; Gottlieb et al. 2022), calling for a common mechanism causing high-velocity wings in this type of object. In the case of EP Aqr, where the bipolar outflow axis almost coincides with the line of sight (with an inclination angle of $i \approx 10^{\circ}$ ), the highvelocity wings were interpreted in terms of narrow polar jets. For R Dor and $o$ Cet, which do not show obvious signs of axial symmetry in their winds, such an interpretation could not be retained and it was argued instead that the high-velocity wings were caused by (a mixture of) turbulence, thermal broadening, and some effect of shocks, acting at distances below some 10 to $15 \mathrm{AU}$ from the central star. The presence of broad wings in the $\mathrm{SiO}$ lines emitted from RS Cnc, whose symmetry axis is inclined by $\approx 30^{\circ}$ with respect to the line of sight (see Sect. 4.1), lends support to the latter type of interpretation and casts serious doubts on the polar jet interpretation proposed earlier for EP Aqr, which shows a morpho-kinematics similar to that of RS Cnc (Nhung et al. 2015b). Indeed, if the broad line widths are present regardless of the orientation of a possible symmetry axis with the line of sight, they must be caused by a mechanism of nondirectional (accounting for the resolving beam) nature. A possible candidate, whose action is limited to the close vicinity of the star, is pulsation-driven shocks that dissipate their energy relatively close to the star and imply positive and negative velocities in the shocked region that can be much higher than the terminal outflow velocity of the wind. Such structures could be explained by the B-type models discussed in Winters et al. (2000b) as presented in Winters et al. (2002); see their Fig. 3. Recent 3D model calculations that self-consistently describe convection and fundamental-mode radial pulsations in the stellar mantle would provide the physical mechanism that leads to the development of such shocks close to the star surface (e.g., Freytag et al. 2017) and could therefore replace the simplified inner boundary condition (the so-called "piston approximation") that was used in the earlier 1D models mentioned above.

In the data presented here, wings at high Doppler velocity are seen in nearly all lines detected with sufficient sensitivity to probe the profile over at least $v_{\mathrm{lsr}, *} \pm 10 \mathrm{~km} \mathrm{~s}^{-1}$. This is illustrated in Fig. 16, where $v_{z}$ profiles are integrated over a circle of radius $0.2^{\prime \prime}$ centered on the star. Gaussian profiles centered at the origin are shown as visual references (not fits), showing how absorption produces asymmetric profiles. A major difference is seen between vibrational ground-state lines, which have a Gaussian FWHM of $\sim 10 \mathrm{~km} \mathrm{~s}^{-1}$, and vibrationally excitedstate lines, which have a Gaussian FWHM of $\sim 14 \mathrm{~km} \mathrm{~s}^{-1}$. Such a difference is not surprising, assuming that the highvelocity wings are formed in the inner layer of the circumstellar envelope (CSE), which is preferentially probed by the $(v=1)$ lines. In this context, we note that Rizzo et al. (2021) recently reported the detection of a narrow $\operatorname{SiO}(v=1,1-0)$ maser line in $\mathrm{RS} \mathrm{Cnc}$ at a velocity of $+14 \mathrm{~km} \mathrm{~s}^{-1}$ with respect to the star's lsr velocity. The effect of shocks on line profiles was first observed in the near-infrared range on $\mathrm{CO}$ ro-vibrational lines, probing the stellar photosphere and the innermost circumstellar region within $\sim 10 R_{*}$ (e.g., $\chi$ Cyg, an S-type star, Hinkle et al. 1982). Very-high-angular-resolution observations obtained over the past decade using VLT, VLTI, and ALMA show that the effect of shocks from pulsations and convection cell ejections is confined within some $10 \mathrm{AU}$ from the star (see, e.g., Khouri et al. 2018; Höfner \& Olofsson 2018; Ohnaka et al. 2019, and references therein). Rotation, when observed, is instead found

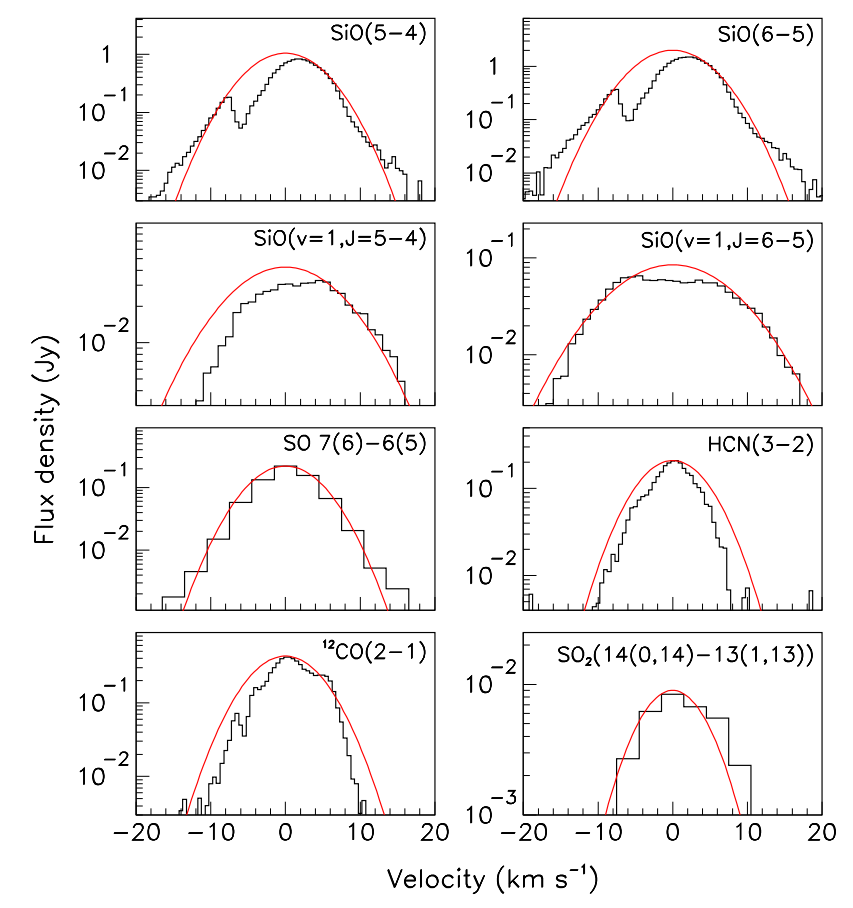

Fig. 16. Line profiles of different molecules on a logarithmic intensity scale. Gaussian profiles are shown for comparison, $F W H M=10 \mathrm{~km} \mathrm{~s}^{-1}$ for the ground-state lines of all molecules, and $F W H M=14 \mathrm{~km} \mathrm{~s}^{-1}$ for the $(v=1)$ lines of $\mathrm{SiO}$. All observed profiles are integrated over $R<0.2^{\prime \prime}$

to extend beyond this distance, typically up to $\sim 20 \mathrm{AU}$ (e.g., Vlemmings et al. 2018; Homan et al. 2018a; Nhung et al. 2021). The angular resolution of the present data is insufficient to detect such differences directly; however, the effect of rotation and shocks on lines of sight contained within a beam centered on the star depends on the region probed by each specific line: lines that probe the inner layers exclusively, such as the $(v=1)$ lines, are mostly affected by shocks, and somewhat by rotation; $\mathrm{CO}$ lines, for which the probed region extends very far out, see little effects of rotation and even less effects of shocks because the emission from the inner envelope provides too small a fraction of the total emission. Between these two extremes, the relative importance of the contributions of shocks and rotation depends on the radial extent of the region probed by the line. Such an interpretation is consistent with the data displayed in Fig. 16.

\subsection{Rotation}

In Fig. 17, we present first-moment maps of $\mathrm{HCN}(3-2)$ (left), $\mathrm{SO}_{2}(14(0,14)-13(1,13))$ (middle left), $\mathrm{SiO}(v=1,6-5)$ (middle right), and $\mathrm{SO}(7(6)-6(5))$ (right). At projected distances from the star not exceeding $0.5^{\prime \prime}$, all four tracers display approximate anti-symmetry with respect to a line at $\mathrm{PA} \sim 10^{\circ}$. This is suggestive of the presence of rotation in the inner CSE layer around an axis that projects on this line in the plane of the sky. Such a morpho-kinematic structure has also been observed in other stars, notably R Dor (Vlemmings et al. 2018; Homan et al. 2018a; Nhung et al. 2021). The angular resolution of the present data does not allow for a detailed exploration of this region, which prevents us from commenting on its possible cause. Nevertheless, the anti-symmetry axis of the velocity pattern projected on the plane of the sky at a PA that approximately coincides with the projected symmetry axis of the polar outflows (see Sect. 4.1) 

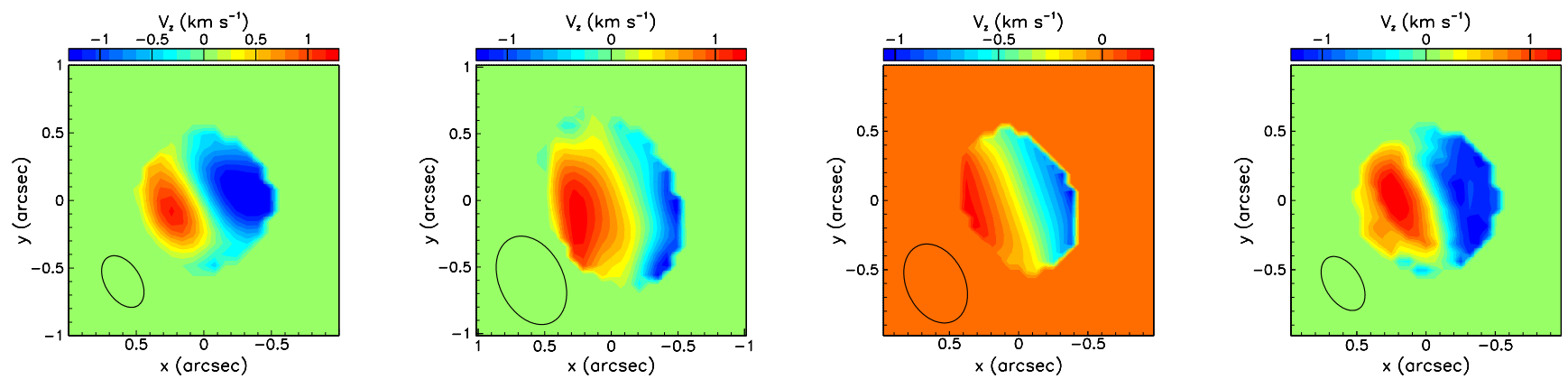

Fig. 17. First-moment maps of different lines, indicating a possibly rotating structure (see Sect. 3.4). Left: $\mathrm{HCN}(3-2)$, middle left: $\mathrm{SO}_{2}(14(0,14)-$ 13(1,13)), middle right: $\mathrm{SiO}(v=1,6-5)$, right: $\mathrm{SO}(7(6)-6(5))$. The black ellipses indicate the synthesized beam.

is remarkable and suggests that rotation is taking place about this same polar axis in the inner CSE layer.

The line-of-sight velocities of these structures are small, on the order of the velocities derived from $\mathrm{CO}$ for the equatorial region, and we interpret them here as possible signs of rotation (rather than indicating another bipolar outflow oriented perpendicular to the larger scale outflow traced in $\mathrm{CO}$ and $\mathrm{SiO}(v=0)$ lines). We note that out of these four lines, the $\mathrm{HCN}(3-2)$ line is detected with the highest $\mathrm{S} / \mathrm{N}(\mathrm{S} / \mathrm{N}=233$ in the line peak, cf. Table 1).

The mean Doppler velocity $\left\langle v_{z}\right\rangle$, averaged over the inner $0.5^{\prime \prime}$, of the HCN line can be fit in position angle $\omega$, measured counter-clockwise from north, by

$\left\langle v_{z}\right\rangle_{\mathrm{HCN}}=-0.19 \mathrm{~km} \mathrm{~s}^{-1}+1.0 \mathrm{~km} \mathrm{~s}^{-1} \sin \left(\omega-19^{\circ}\right)$,

whereas the $\operatorname{SiO}(v=1,6-5)$ velocity is well fit by

$\left\langle v_{z}\right\rangle_{\mathrm{SiO}(v=1,6-5)}=-0.37 \mathrm{~km} \mathrm{~s}^{-1}+0.46 \mathrm{~km} \mathrm{~s}^{-1} \sin \left(\omega-26^{\circ}\right)$.

The small offsets of $\sim-0.3 \mathrm{~km} \mathrm{~s}^{-1}$ on average are within the uncertainty attached to the measurement of the star's LSR velocity. The coefficients of the sine terms measure the projected rotation velocity, namely the rotation velocity divided by the sine of the angle made by the rotation axis with the line of sight. Assuming that the rotation axis is the axi-symmetry axis of the CSE, this angle is $i \sim 30^{\circ}$ (see Sect. 4.1), meaning rotation velocities of $\sim 2$ and $\sim 1 \mathrm{~km} \mathrm{~s}^{-1}$ for $\mathrm{HCN}$ and $\mathrm{SiO}$ respectively. Observations of higher angular resolution are needed to confirm the presence of rotation within a projected distance of $0.5^{\prime \prime}$ from the star and we prefer to summarize the results presented in this section in the form of an upper limit to the mean rotation velocity of a few $\mathrm{km} \mathrm{s}^{-1}$.

\subsection{Global outflow structure traced by $\mathrm{CO}$ and $\mathrm{SiO}$}

The detailed structure of the morpho-kinematics of the CSE has been studied using observations of the ${ }^{12} \mathrm{CO}(1-0)$ and ${ }^{12} \mathrm{CO}(2-1)$ molecular line emission. The analyses of Hoai et al. (2014) and Nhung et al. (2015b) confirmed the interpretation of the twocomponent nature of the Doppler velocity spectrum originally given by Libert et al. (2010). The CSE is axi-symmetric about an axis making an angle of $i \sim 30^{\circ}$ with the line of sight and projecting on the plane of the sky at a position angle $\omega \sim 7^{\circ}$ east of north (see also the sketch in Fig. 4). The expansion velocity reaches $\sim 8$ to $9 \mathrm{~km} \mathrm{~s}^{-1}$ along the axis - we refer to this part of the CSE as bipolar outflow - and $\sim 3$ to $4 \mathrm{~km} \mathrm{~s}^{-1}$ in the plane perpendicular to the axis - we refer to this part of the CSE as equatorial enhancement. The transition from the equator to the poles of the CSE is smooth. Section 4.1 below, using observations of the ${ }^{12} \mathrm{CO}(2-1)$ and ${ }^{13} \mathrm{CO}(2-1)$ molecular lines, confirms and significantly refines this picture. The right panels of Fig. 20 show projections of the CSE on the plane containing the axis and perpendicular to the plane of the sky, which give a good qualitative idea of the global structure.

Velocity-integrated channel maps of the $\mathrm{CO}(2-1)$ and $\mathrm{SiO}(6-5)$ observations analyzed in the present article are displayed in Fig. 18. They clearly show the bipolar outflows, inclined toward the observer in the north and receding in the south. We note that the red wings are brighter than the blue wings as a result of absorption (see Sects. 4.1 and 4.2) The SiOemitting region is seen to be significantly more compact than the CO-emitting region; this is in conformity with observations of many other oxygen-rich AGB stars and is generally interpreted as the result of $\mathrm{SiO}$ molecules condensing on dust grains and being ultimately dissociated by the interstellar radiation at some $200 \mathrm{AU}$ from the star, well before CO molecules are dissociated (see e.g., Schöier et al. 2004).

\subsection{Temperature and $\mathrm{SO}_{2}$ abundance}

In this section, we use the 11 detected $\mathrm{SO}_{2}$ lines to derive an approximate temperature and column density of the $\mathrm{SO}_{2}$ emitting region by means of a population diagram. Following Goldsmith \& Langer (1999), in the optically thin case, the column density of the upper level population $N_{u}$ of a transition $u->l$ can be expressed as

$N_{u}=\frac{8 \pi \mathrm{k} v^{2}}{\mathrm{~h} c^{3} A_{u l}} \int T_{b} \mathrm{~d} v$.

$N_{u}$ is the column density of the upper level population of the transition, $k$ and $h$ are the Boltzmann and Planck constant, respectively, $v$ is the line frequency, $c$ the speed of light, $A_{u l}$ is the Einstein coefficient for spontaneous emission of the transition, and $\int T_{b} \mathrm{~d} v$ is the velocity-integrated main-beam brightness temperature. The latter is converted to the surface brightness distribution of the source $S_{v}$ per beam, measured by the interferometer, by means of

$T_{b}=\frac{\lambda^{2}}{2 k \Omega_{b}} S_{v}$,

where $\lambda=\frac{c}{v}$ is the observing wavelength, and $\Omega_{b}=\frac{\pi \theta_{a} \theta_{b}}{4 \ln 2}$ with $\theta_{a}$ and $\theta_{b}$ being the major and minor axis of the synthesized beam.

We determine $\int S(v) \mathrm{d} v=: W_{I}$ from a circular Gaussian fit to the velocity-integrated emission in the $u v$-plane, where the integration is taken from $\left(v_{\mathrm{lsr}, *}-4.5\right) \mathrm{km} \mathrm{s}^{-1}$ to $\left(v_{\mathrm{lsr}, *}+4.5\right) \mathrm{km} \mathrm{s}^{-1}$, that is over the three central channels of the $\mathrm{SO}_{2}$ lines. 


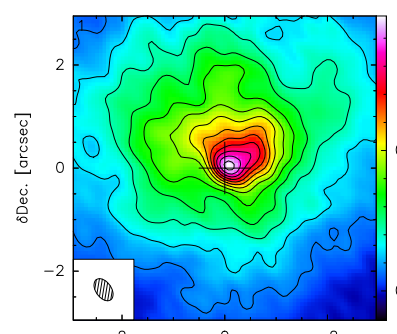

6R.A. [arcsec]

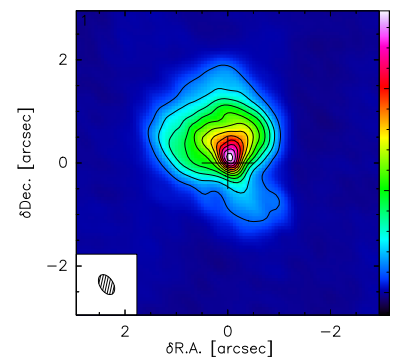

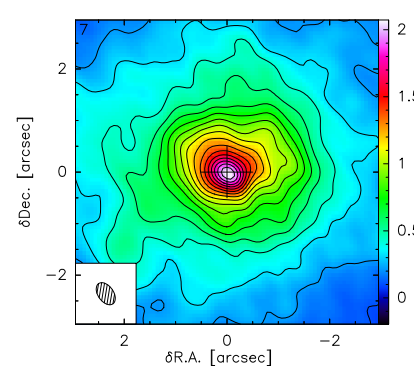

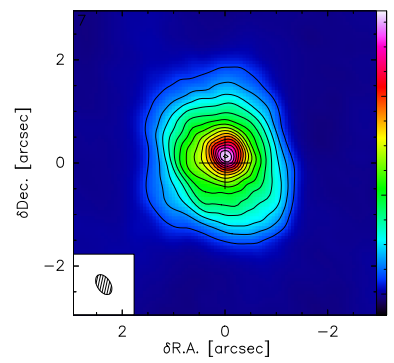

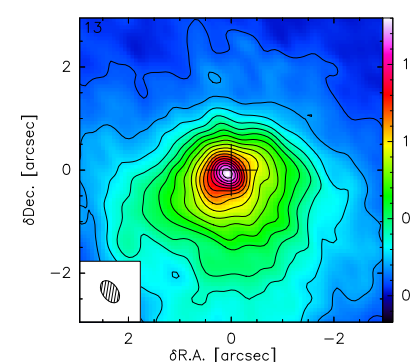

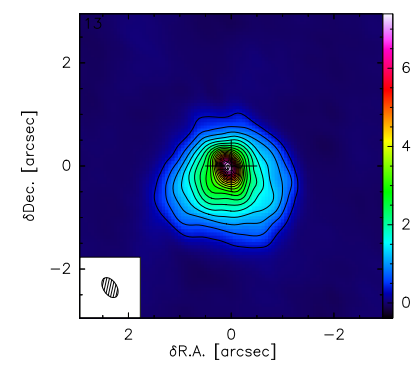

Fig. 18. Velocity-integrated intensity maps of the ${ }^{12} \mathrm{CO}(2-1)$ line (upper row) and the vibrational ground-state line of $\mathrm{SiO}(6-5)$ (lower row), covering three velocity intervals. Left: blue line wing $\left[v_{\mathrm{lsr}, *}-10, v_{\mathrm{lsr}, *}-2\right] \mathrm{km} \mathrm{s}^{-1}$, middle: line center $\left[v_{\mathrm{lsr}, *}-2, v_{\mathrm{lsr}, *}+2\right] \mathrm{km} \mathrm{s}^{-1}$, right: red line wing $\left[v_{\mathrm{ls}, *}+2, v_{\mathrm{lsr}, *}+10\right] \mathrm{km} \mathrm{s}^{-1}$. North is up and east is to the left. We note the different color scales. Contours are plotted every $5 \sigma$ for CO and every $20 \sigma$ for $\mathrm{SiO}$, where (from left to right) $1 \sigma=14.6,22.0,19.2 \mathrm{mJy} \mathrm{beam}^{-1} \mathrm{~km} \mathrm{~s}^{-1}$ for $\mathrm{CO}(2-1)$ and $1 \sigma=11.1,16.7,16.4 \mathrm{mJy} \mathrm{beam}^{-1} \mathrm{~km} \mathrm{~s}^{-1}$ for $\mathrm{SiO}(6-5)$. The black ellipse in the lower left corner indicates the synthesized beam.

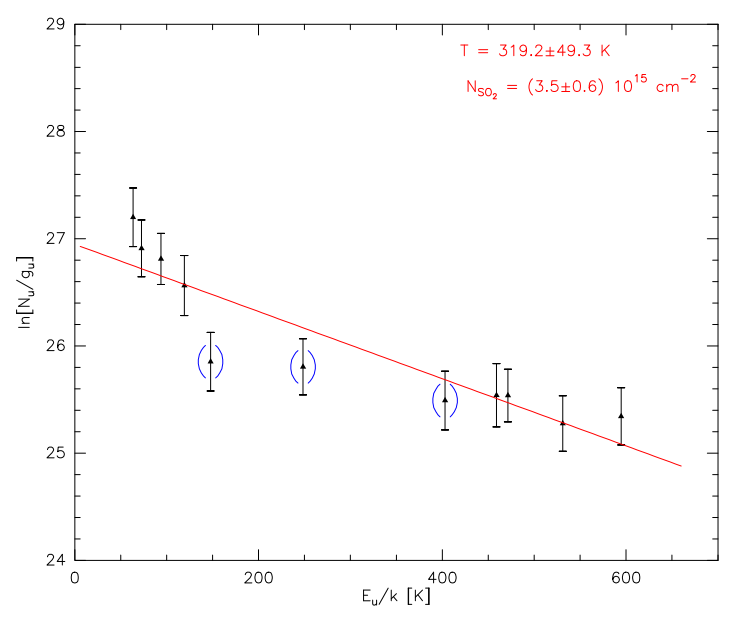

Fig. 19. Population diagram for $\mathrm{SO}_{2}$. The three data points in brackets correspond to the three lowest frequency $\mathrm{SO}_{2}$ lines observed with setup 1 , which, due to a different $u v$ coverage, resulted in a comparably larger beam than the setup 2 observations (see Table 2 and Eq. (6)).

For the population diagram, we then get

$\ln \left(\frac{N_{u}}{g_{u}}\right)=\ln \left(\frac{\zeta_{u} W_{I}}{g_{u}}\right)=\ln \left(\frac{N_{\mathrm{SO}_{2}}}{Q_{\mathrm{SO}_{2}, \mathrm{rot}}}\right)-\frac{E_{u}}{k T}$,

where we define $\zeta_{u}$ as

$\zeta_{u}=\frac{4.784 \times 10^{-7}}{\mathrm{~h} c A_{u l}} \frac{1}{\theta_{a} \theta_{b}}$.

In Eq. (5), $W_{I}$ is expressed in $\mathrm{Jy} \times \mathrm{km} \mathrm{s}^{-1}, \theta_{a}$ and $\theta_{b}$ are given in $\operatorname{arcsec}, E_{u}$ is the upper level energy, $g_{u}$ the statistical weight of the upper level, and $T$ is the excitation temperature. All relevant parameters of the $\mathrm{SO}_{2}$ transitions used here are listed in Table 2.
In fitting a straight line to the population diagram (shown in Fig. 19) to determine a rotational temperature according to Eq. (5), we assume that the $\mathrm{SO}_{2}$ level populations are dominated by collisions, i.e., that LTE holds for the rotational excitation of $\mathrm{SO}_{2}$, and that the lines are optically thin. The assumption of LTE populations may be questionable for $\mathrm{SO}_{2}$ (see Danilovich et al. 2016), and essentially could result in underestimation of the kinetic gas temperature in the $\mathrm{SO}_{2}$-emitting region (cf. the discussion in Goldsmith \& Langer 1999, their Sect. 5). The effect on the derived column density is more difficult to assess without a detailed non-LTE modeling. However, we note that our result is consistent with the $\mathrm{SO}_{2}$ abundance derived for similar objects by means of a comprehensive non-LTE description (see below). On the other hand, the assumption that the lines are optically thin is justified by the results of our XCLASS modeling; see below and Appendix D.

With the temperature of $T \approx 320 \mathrm{~K}$ resulting from a linear fit to the population diagram shown in Fig. 19, we get the partition function $Q_{\mathrm{SO}_{2} \text {,rot }}$ (interpolated from values given in the CDMS), from which an effective $\mathrm{SO}_{2}$ column density of $N_{\mathrm{SO}_{2}}=3.5 \times 10^{15} \mathrm{~cm}^{-2}$ is determined by means of Eq. (5). The $\mathrm{SO}_{2}$ source is compact (see Table 1 and Fig. B.4, FWHP $\approx 0.5^{\prime \prime}$, corresponding to $75 \mathrm{AU}$, or $\approx 70 R_{*}$ ), which is consistent with the estimated temperature in this inner (possibly rotating) region. Assuming a mass-loss rate of $1 \times 10^{-7} M_{\odot} \mathrm{yr}^{-1}$ in the equatorial region (cf. Sect. 4.1), an outflow velocity of at maximum $8 \mathrm{~km} \mathrm{~s}^{-1}$ as indicated by the line widths (but excluding the high-velocity wings discussed in Sect. 3.3), an inner radius of $\approx 10^{14} \mathrm{~cm}$, and an outer radius of that region of $\approx 10^{15} \mathrm{~cm}$, corresponding to $0.5^{\prime \prime}$, we estimate upper limits of the $\mathrm{SO}_{2}$ abundance of $X\left(\mathrm{SO}_{2} /<\mathrm{H}>\right)=7.3 \times 10^{-7}$, or, if hydrogen were completely bound in $\mathrm{H}_{2}, X\left(\mathrm{SO}_{2} / \mathrm{H}_{2}\right)=1.5 \times 10^{-6}$. For comparison, Danilovich et al. (2016) find an $\mathrm{SO}_{2}$ abundance of $\sim 5 \times 10^{-6}$ for the low-mass-loss-rate $\left(\sim 1-2 \times 10^{-7} M_{\odot} \mathrm{yr}^{-1}\right)$ oxygen-rich stars R Dor and W Hya, about a factor of approximately 3 higher than the value found here for the MS-type star RS Cnc. We note 

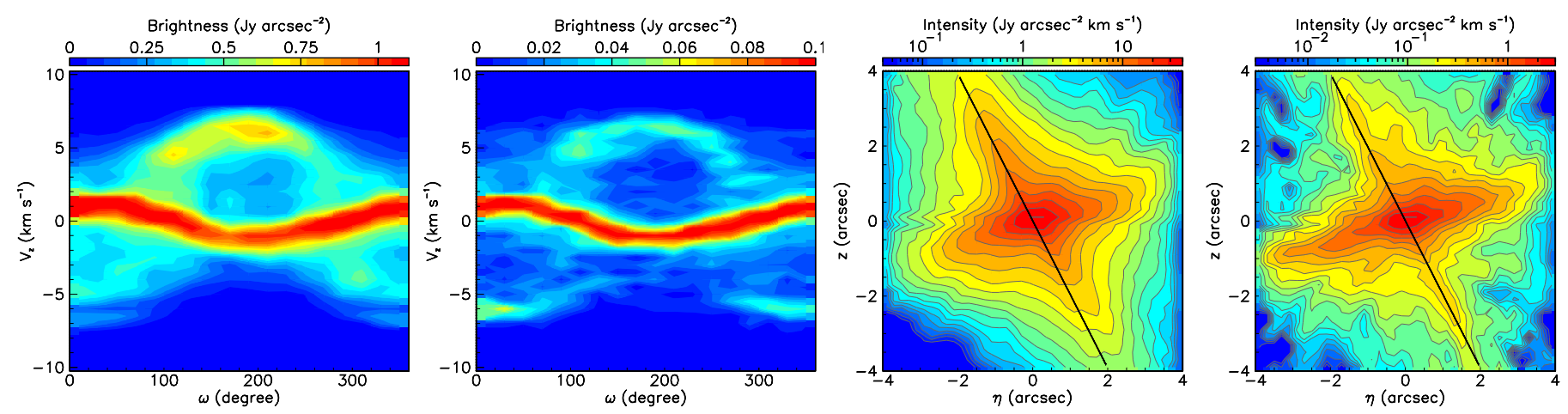

Fig. 20. CO data and 3D reconstruction. Left panels: flux density averaged over $0.4^{\prime \prime}<R<2.5^{\prime \prime}$ in the $v_{z}$ vs. $\omega$ plane for ${ }^{12} \mathrm{CO}(2-1)($ left $)$ and ${ }^{13} \mathrm{CO}(2-1)$ (center-left). Right panels: de-projected flux density projected on the plane perpendicular to the sky and containing the polar outflow axis, shown as black lines, for ${ }^{12} \mathrm{CO}(2-1)$ (center-right) and ${ }^{13} \mathrm{CO}(2-1)$ (right).

that Decin et al. (2008) and De Beck \& Olofsson (2020) do not detect $\mathrm{SO}_{2}$ in the S-type star W Aql. Our result therefore appears to be consistent with an intermediate chemical state of RS Cnc.

In Appendix D, an XCLASS modeling is presented that results in the same $\mathrm{SO}_{2}$ column density of $3.5 \times 10^{15} \mathrm{~cm}^{-2}$ as derived from the population diagram, and in a slightly higher rotational temperature of $350 \mathrm{~K}$, still within the uncertainties of $\sim \pm 50 \mathrm{~K}$ derived here (see Fig. 19). The XCLASS modeling of the $11 \mathrm{SO}_{2}$ lines results in an average optical depth of 0.1 , confirming that the lines are optically thin.

\section{Discussion}

\section{1. ${ }^{12} \mathrm{CO}(2-1)$ and ${ }^{13} \mathrm{CO}(2-1)$ : morpho-kinematics of the circumstellar envelope}

Rotational CO lines in the vibrational ground state are known to probe the circumstellar envelope of AGB stars up to distances on the $1000 \mathrm{AU}$ scale, where the $\mathrm{CO}$ molecules are dissociated by the interstellar UV radiation (Mamon et al. 1988). In this section, we use observations of the ${ }^{12} \mathrm{CO}(2-1)$ and ${ }^{13} \mathrm{CO}(2-1)$ lines to probe the morpho-kinematics of the wind at distances from the star in excess of $\sim 50 \mathrm{AU}$, in a region where the wind is expected to evolve smoothly toward the constant expansion regime.

Both ${ }^{12} \mathrm{CO}(2-1)$ and ${ }^{13} \mathrm{CO}(2-1)$ data have a Doppler velocity $\left(v_{z}\right)$ spectrum that clearly shows a two-component structure, where the blueshifted wing of the ${ }^{12} \mathrm{CO}$ line is partly absorbed, very similar to the corresponding situation in EP Aqr (TuanAnh et al. 2019). CO rotational lines from higher J levels (5-4 and 9-8) observed with Herschel (Danilovich et al. 2015) show triangular profiles consistent with the double-component wind structure that we are proposing.

In the spatial distribution of the $\mathrm{CO}$ emission, a clear separation is observed between the equatorial region and the polar outflows. This is illustrated in Fig. 20, where we show the maps of the flux density in the Doppler velocity $\left(v_{z}\right)$ versus position angle $(\omega)$ plane. The flux density is integrated over an interval of projected distance $(R)$ from the star between $0.4^{\prime \prime}$ and $2.5^{\prime \prime}$. The equatorial region is seen as an intense oscillation at low values of $\left|v_{z}\right|$ while the polar outflows are seen as emission at larger $\left|v_{z}\right|$ values, in phase opposition. From these maps, we estimate, in agreement with earlier findings, that the outflow axis projects on the plane of the sky at a position angle of $\omega=7^{\circ} \pm 5^{\circ}$, at which the equatorial and bipolar outflow oscillations are maximal and minimal, respectively. Figure 20 also shows de-projections of the data cubes projected on the plane perpendicular to the plane of the sky that contains the polar outflow axis. These are drawn assuming a dependence of the wind velocity on stellar latitude $\alpha$ of the form $v_{\text {term }}=4+5 \sin ^{4} \alpha \mathrm{km} \mathrm{s}^{-1}$, increasing from 4 to $9 \mathrm{~km} \mathrm{~s}^{-1}$ from the equator to the poles. The velocity field is assumed to be radial and to have reached its terminal value in the $R$ interval considered here.

In order to interpret these observations, in particular possible differences in the ${ }^{12} \mathrm{CO} /{ }^{13} \mathrm{CO}$ ratio between the equatorial region and polar outflows, we need to take into account the effect of absorption. We choose to consider only regions outside $0.4^{\prime \prime} \sim 60 \mathrm{AU}$ from the star, which allows for major simplifications: we can ignore the very complex kinematics that governs the inner CSE layers, including shocks and possible rotation. As we cannot expect the data to strongly constrain the temperature distribution, we take it of the form $T=T_{0} \exp \left(-r / r_{0}\right)$ and fit the observed data cubes by means of a $\chi^{2}$ minimization scheme for different values of the $\left(T_{0}, r_{0}\right)$ pair consistent with earlier estimates (Nhung et al. 2015a). We find that the morpho-kinematics is best described by modeling the polar outflows and equatorial region separately, with a separation at stellar latitude $\alpha_{0} \sim 30^{\circ}$. We parametrize the radial dependence of the wind velocity as $v=v_{\text {term }} r /\left(r+r_{1 / 2}\right)$ : it increases from 0 at $r=0$ to $v_{\text {term }}$ at $r=\infty$, reaching $\frac{1}{2} v_{\text {term }}$ at $r=r_{1 / 2}$. In order to account for different opening angles of the polar outflows and different flaring angles of the equatorial enhancement, we allow, within each region, for latitudinal dependencies of the velocity described as Gaussian functions in $\sin \alpha$ in the equatorial region and in $\cos \alpha$ in the polar outflows with adjustable Gaussian widths $\sigma$.

We do the same for the number densities, which vary radially as $\rho_{0}\left(v_{\text {term }} / v\right) r^{-2}$ with different $\rho_{0}$ values for the equatorial region and the polar outflows, and of course for ${ }^{12} \mathrm{CO}$ and ${ }^{13} \mathrm{CO}$. The radiative transfer equation is integrated in a sphere of $10^{\prime \prime}$ radius and the calculated flux densities are then smeared with the synthesized beam of the observations. Reasonably good fits of the data cubes are obtained given the crudeness of the model (see Fig. 21).

We find that the best fits to the morpho-kinematics of the two lines are relatively insensitive to the exact form assumed for the temperature structure. For $r_{0}=2^{\prime \prime}$, we find that values of $T_{0}$ between $70 \mathrm{~K}$ and $170 \mathrm{~K}$ in the equatorial region and between $100 \mathrm{~K}$ and $200 \mathrm{~K}$ in the polar outflows are acceptable. Changing the value of $r_{0}$ modifies the values of $T_{0}$ accordingly but does not improve the quality of the fit. Parameters of the best-fit model include an angle of the polar outflow axis with the line of sight $i \approx 30^{\circ}$ as expected, a separation in latitude between polar and equatorial regions, $\alpha_{0}=29 \pm 4 \mathrm{deg}$, terminal velocities of 

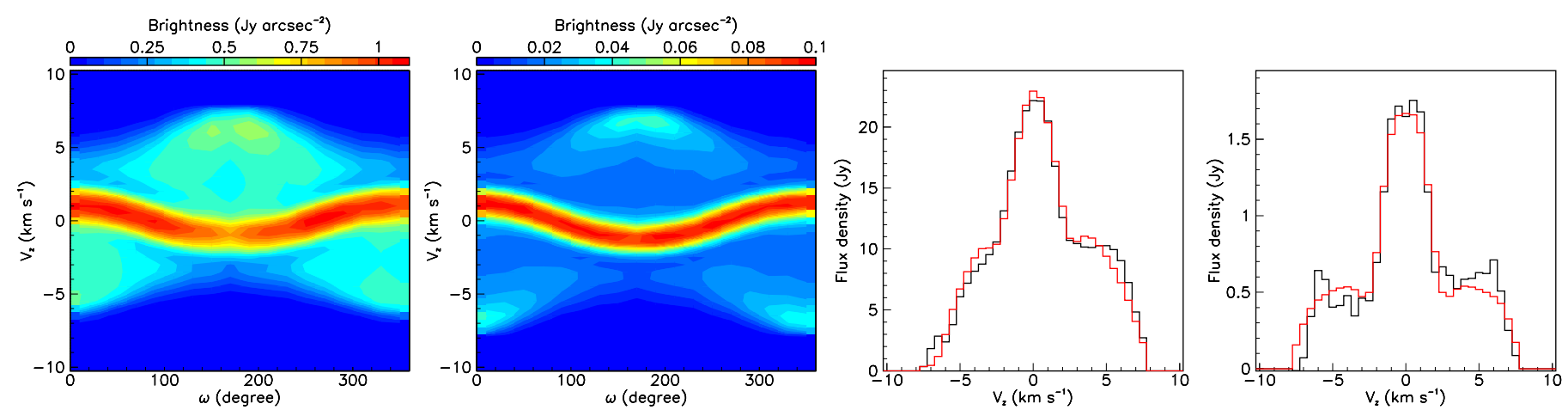

Fig. 21. Best-fit results for CO. Left panels: same as in Fig. 20, but for the model best fit. Right panels: modeled $v_{z}$ spectra (red) compared with observations (black) for ${ }^{12} \mathrm{CO}(2-1)$ (center-right) and ${ }^{13} \mathrm{CO}(2-1)$ (right).

Table 3. Parameters of the best-fit model for CO.

\begin{tabular}{cccccccccc}
\hline \hline Region & $\begin{array}{c}v_{\text {term }} \\
\left(\mathrm{km} \mathrm{s}^{-1}\right)\end{array}$ & $\begin{array}{c}r_{1 / 2} \\
(\mathrm{arcsec})\end{array}$ & $\begin{array}{c}T_{0} \\
(\mathrm{~K})\end{array}$ & $\begin{array}{c}r_{0} \\
(\mathrm{arcsec})\end{array}$ & $\begin{array}{c}\text { Opening angle } \\
(\mathrm{FWHM})(\mathrm{deg})\end{array}$ & $\sigma(\rho)$ & $\sigma(v)$ & $\begin{array}{c}\rho_{0}\left({ }^{12} \mathrm{CO}\right) \\
\left(\mathrm{cm}^{-3}\right)\end{array}$ & $\begin{array}{c}\rho_{0}\left({ }^{13} \mathrm{CO}\right) \\
\left(\mathrm{cm}^{-3}\right)\end{array}$ \\
\hline Equator & $3.5 \pm 0.7$ & $0.6 \pm 0.2$ & 100 & 2.0 & 30 & $0.21 \pm 0.03$ & $>0.4$ & $86 \pm 10$ & $4.2 \pm 0.4$ \\
Poles & $8.6 \pm 0.5$ & $0.29 \pm 0.10$ & 130 & 2.0 & 70 & $0.50 \pm 0.02$ & $0.70 \pm 0.04$ & $70 \pm 6$ & $2.9 \pm 0.4$ \\
\hline
\end{tabular}

Notes. The quoted uncertainties correspond to a $10 \%$ increase in the best fit $\chi^{2}$, leaving all other parameters fixed at their best-fit value: they should not be understood as uncertainties but as indicators of the sensitivity of the quality of the fit to each separate parameter.

$3-4 \mathrm{~km} \mathrm{~s}^{-1}$ in the equatorial region, and $8-9 \mathrm{~km} \mathrm{~s}^{-1}$ in the polar outflows, outflow opening angle and equatorial flaring angle of $\sim 70^{\circ}$ and $\sim 30^{\circ}$, respectively $(F W H M=2.35 \times \arccos (\sigma(\rho))$ and $2.35 \times \arcsin (\sigma(\rho))$, respectively); these are listed in Table 3 . The wind is still being accelerated (the escape velocity of a $1.5 M_{\odot}$ star at a distance of $150 \mathrm{AU}\left(\approx 1^{\prime \prime}\right.$ for $\left.\mathrm{RS} \mathrm{Cnc}\right)$ is $4.2 \mathrm{~km} \mathrm{~s}^{-1}$ ) - having reached half terminal velocity at the inner edge of the observed radial range - earlier in the poles than in the equatorial region. The number densities of $\mathrm{CO}$ molecules correspond to mass-loss rates of $1.0 \times 10^{-7} M_{\odot} \mathrm{yr}^{-1}$ in the equatorial region and $2.0 \times 10^{-7} M_{\odot} \mathrm{yr}^{-1}$ in the outflows ${ }^{6}$ for a $\mathrm{CO} / \mathrm{H}_{2}$ ratio of $2 \times 10^{-4}$. The ${ }^{12} \mathrm{CO} /{ }^{13} \mathrm{CO}$ ratio is measured to be $\sim 20$ on average, but larger in the polar outflows $(24 \pm 2)$ than in the equatorial region $(19 \pm 3)$. This is a barely significant difference, but a similar asymmetry seems to be present in EP Aqr (Tuan-Anh et al. 2019): such a result is unexpected and needs to be confirmed by higher sensitivity observations before being accepted. Indeed, if it were confirmed, this might suggest that the polar outflows are fed in part from material freshly produced in the 3- $\alpha$ process and mixed into the atmosphere by the third dredge-up following a He shell flash. This process would not only increase the ${ }^{12} \mathrm{C}$ abundance in the atmosphere, but also the ${ }^{12} \mathrm{C} /{ }^{13} \mathrm{C}$ isotope ratio, as indicated for example by Smith \& Lambert (1990); see in particular their Fig. 9.

The presence of an equatorial density enhancement with a rather small flaring angle, $\sim 30^{\circ} \mathrm{FWHM}$, suggests that it may rather be a disk, which might be expected to be rotating and to have an inner rim. However, the size of the beam is too large to study this reliably. From a close inspection of the $\left\langle v_{z}\right\rangle$ distribution near the star, using the method described in Sect. 3.4

6 This is about a factor 2 larger than the values quoted in Hoai et al. (2014). Their data were affected by a pointing offset of the old $30 \mathrm{~m}$ OTF maps that lead to an underestimation of the CO line flux of about a factor 2 . for $\mathrm{HCN}$ and $\mathrm{SiO}$, we infer a rotation velocity at $r \sim 0.5^{\prime \prime}$ of $v_{\text {rot }}=\left|v_{z}\right| / \sin i \sim 2.5 \mathrm{~km} \mathrm{~s}^{-1}$. However, this is a very crude estimate given the size of the beam and the lack of precise knowledge of the morpho-kinematics in the innermost radial range.

The blob of enhanced ${ }^{12} \mathrm{CO}(2-1)$ line emission that had been identified in Hoai et al. (2014) as possibly suggesting the presence of a companion is seen on the channel maps in the $v_{\mathrm{lsr}}=6$ to $7 \mathrm{~km} \mathrm{~s}^{-1}$ range as an elongation in the west-northwest to eastsoutheast direction (Fig. 22). Projections of the data cube on different planes ( $v_{z}$ vs. $\omega, v_{z}$ vs. $R$, and $\omega$ vs. $R$ ) in its neighborhood show that it can be described as a pair of elongations at position angles of $\sim 120^{\circ}$ and $\sim 270^{\circ}$, the latter being significantly more intense than the former and covering a broad range of $R$ between 1 and $2^{\prime \prime}$. While these features provide no justification for a possible identification of a companion, they cannot be used either as arguments against the presence of an unobserved companion.

\section{2. $\mathrm{SiO}(5-4)$ and $\mathrm{SiO}(6-5)$ : evidence for strong absorption}

In the present section, we compare the $\mathrm{SiO}(5-4)$ and $\mathrm{SiO}(6-5)$ line emission with the ${ }^{12} \mathrm{CO}$ results described in the previous section. In contrast to $\mathrm{CO}$, the $\mathrm{SiO}$ emission does not resolve the equatorial region from the polar outflows, as illustrated in the leftmost panels of Fig. 23. Part of the reason for this is the much smaller radial range being probed, as illustrated in the right panel of Fig. 23. As mentioned earlier in Sect. 3.5, the radial extent of the $\mathrm{SiO}$ emission is often significantly smaller than that of the $\mathrm{CO}$ emission, which is usually interpreted as evidence for the progressive condensation of $\mathrm{SiO}$ molecules on dust grains (e.g., Schöier et al. 2004). This would cause the progressive decline of the $\mathrm{SiO} / \mathrm{CO}$ ratio observed in the right panel of Fig. 23 up to $R \sim 1.5^{\prime \prime}$, followed by a more abrupt cut-off around $R \sim 2^{\prime \prime}$ 

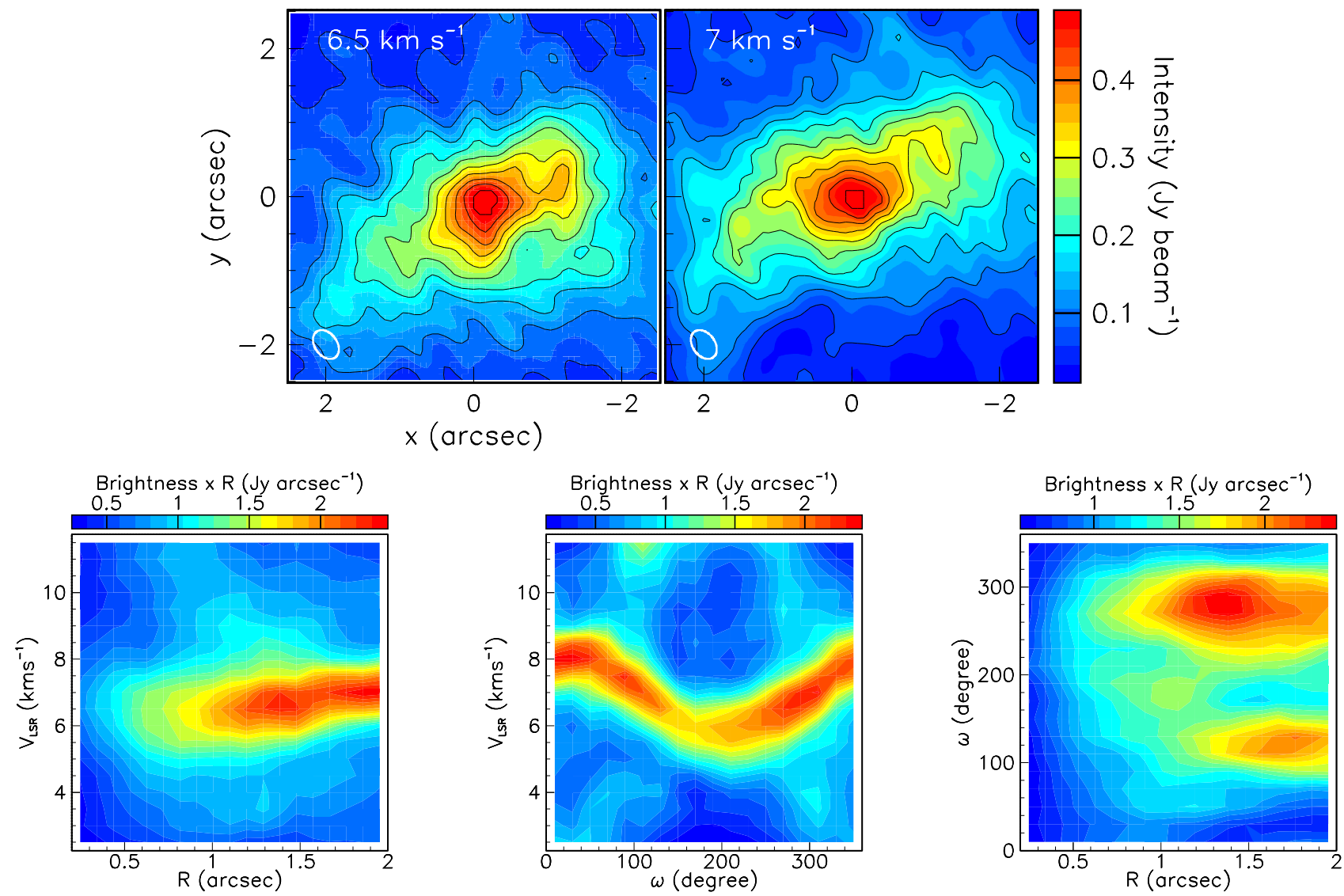

Fig. 22. Enhanced ${ }^{12} \mathrm{CO}(2-1)$ emission at $v_{\mathrm{lsr}} \sim 6.75 \mathrm{~km} \mathrm{~s}^{-1}$. Upper panels: channel maps of the ${ }^{12} \mathrm{CO}(2-1)$ line emission between 6.25 and $7.25 \mathrm{~km} \mathrm{~s}^{-1}$. Lower panels: projection of the flux density multiplied by $R$ on the $v_{\mathrm{lsr}} \mathrm{vs}$. $R$ plane for $250^{\circ}<\omega<330^{\circ}$ (left), on the $v_{\mathrm{lsr}} \mathrm{vs}$. $\omega$ plane for $0.5<R<2 \operatorname{arcsec}$ (middle), and on the $\omega$ vs. $R$ plane for $5.75<v_{\text {lsr }}<7.25 \mathrm{~km} \mathrm{~s}^{-1}$ (right).
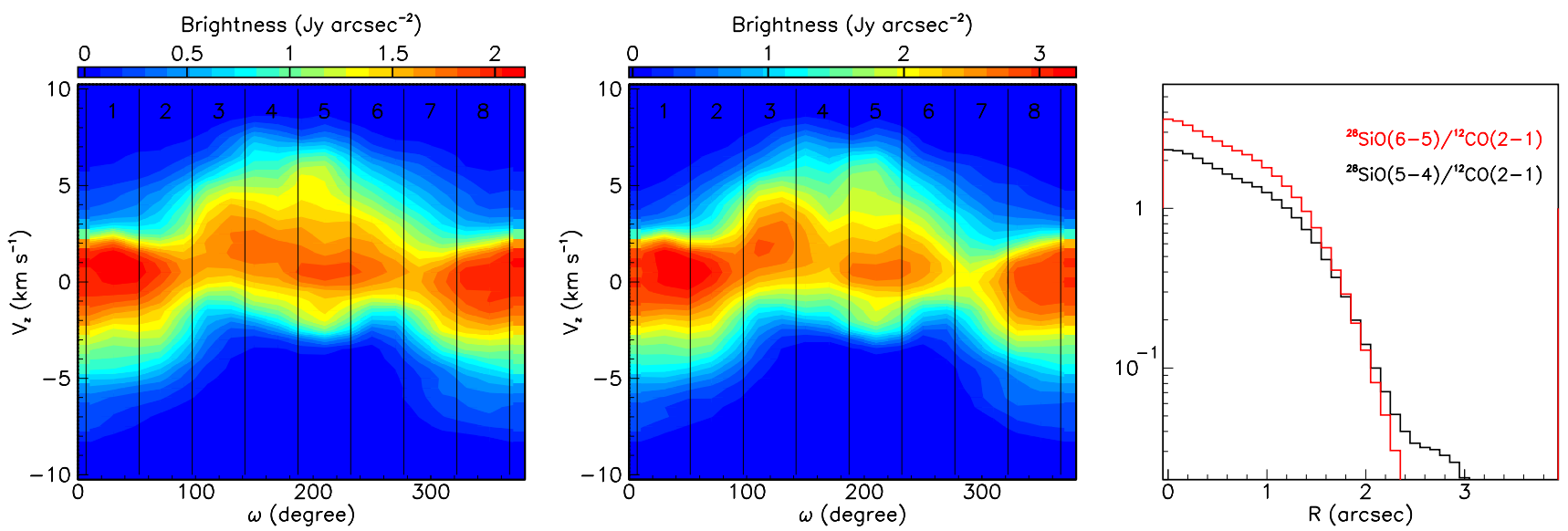

Fig. 23. $v_{z}$ vs. $\omega$ maps for $0.5^{\prime \prime}<R<1.5^{\prime \prime}$. Left: $\mathrm{SiO}(5-4)$, middle: $\mathrm{SiO}(6-5)$, octant intervals in $\omega$ as discussed in the text are indicated. Right: intensity ratio $\mathrm{SiO}(5-4) /{ }^{12} \mathrm{CO}(2-1)$ (black) and $\mathrm{SiO}(6-5) /{ }^{12} \mathrm{CO}(2-1)$ (red) as a function of $R$ for $\left|v_{z}\right|<8 \mathrm{~km} \mathrm{~s}^{-1}$.

caused by the dissociation of the $\mathrm{SiO}$ molecules by the interstellar UV radiation.

Another striking difference is the presence of high-velocity wings in the $\mathrm{SiO}$ data close to the star, that are mostly absent in the CO data (see Figs. 24 and 16). This may be understood as the $\mathrm{SiO}$ data probing the close neighborhood of the star much more efficiently, where pulsation shocks and possibly convection cells are known to play an important role, as demonstrated by a host of observations at shorter wavelengths, from far-infrared to near-UV, and in agreement with theoretical modeling (see, e.g.,
Hinkle et al. 1982; Winters et al. 2000a; Richter et al. 2003; Nowotny et al. 2005; Freytag et al. 2017; Montez et al. 2017; Höfner \& Olofsson 2018, and references therein). Also worth noting in Fig. 24 is the significant absorption in front of the stellar disk seen in the $\mathrm{SiO}$ data at $\sim-6 \mathrm{~km} \mathrm{~s}^{-1}$, reminiscent of similar observations in stars such as R Dor (Nhung et al. 2021), W Hya (Takigawa et al. 2017), and $o$ Ceti (Wong et al. 2016). Of these three stars, two are found to be surrounded by an optically thick $\mathrm{SiO}$ layer that extends well beyond the stellar disk, while in the third, $o$ Ceti, $\mathrm{SiO}$ emission decreases abruptly 

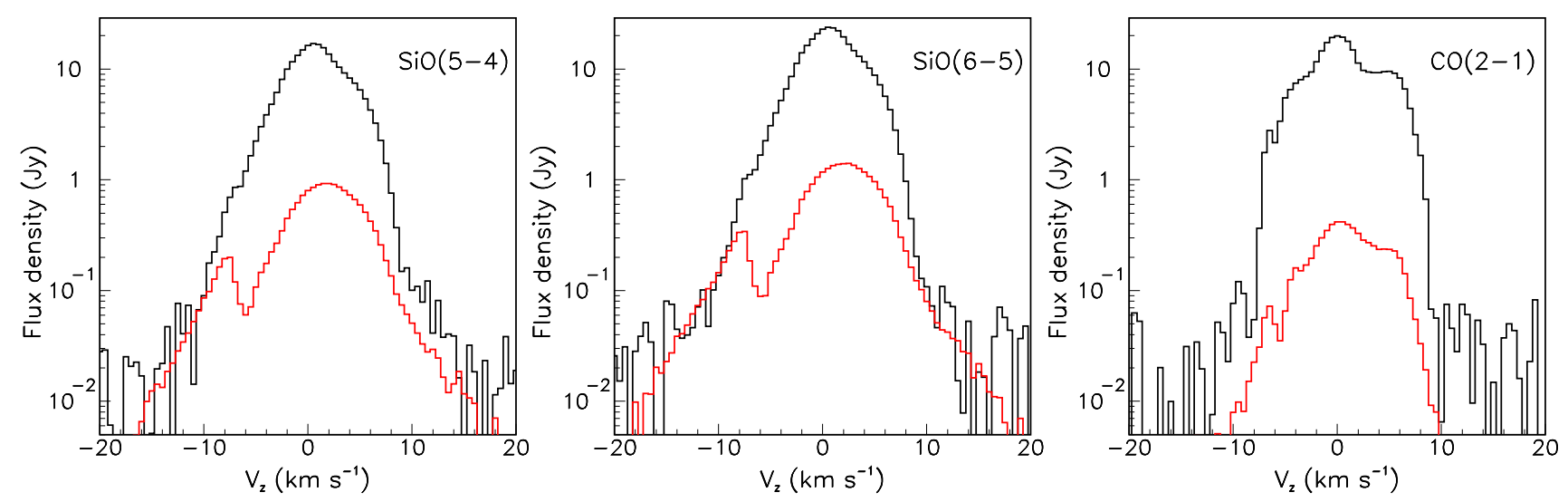

Fig. 24. Doppler velocity spectra integrated over $0.2<R<2.5$ arcsec (black) and within $R<0.2 \operatorname{arcsec}\left(\right.$ red) for $\mathrm{SiO}(5-4)$, $\mathrm{SiO}(6-5)$, and ${ }^{12} \mathrm{CO}(2-$ 1) from left to right.

beyond some 10 to $50 \mathrm{AU}$ from the center of the star. In order to understand the mechanism of condensation of $\mathrm{SiO}$ molecules on dust grains, it is therefore important to study the $\mathrm{SiO}$ emission region in oxygen-rich AGB stars. Accordingly, we devote the remainder of the present section to this task. In a first part, we make some qualitative comments that help with unraveling the general picture; in a second part, we account for radiative transfer using the model of the morpho-kinematics developed for $\mathrm{CO}$ emission in the preceding section. The morpho-kinematics of the $\mathrm{SiO}(5-4)$ and $\mathrm{SiO}(6-5)$ lines are so similar that we cannot expect their comparison to be very sensitive to temperature. At temperature $T$, in the optically thin LTE approximation, the ratio $R_{T}$ of the $\mathrm{SiO}(5-4) / \mathrm{SiO}(6-5)$ line emission depends only on the ratio of the Einstein coefficients, $5.2 \times 10^{-4} / 9.1 \times 10^{-4}=0.57$, and the ratio of the level populations, $(2 J+1)\left(\exp \left(-E_{u} / T\right)\right.$, where the upper level energies $E_{u}$ are $31.3 \mathrm{~K}$ and $43.8 \mathrm{~K}$, respectively (line parameters from the CDMS, based on Müller et al. 2013).

Hence, we have

$R_{T}=0.48 \exp (-31.3 / T) / \exp (-43.8 / T)$

from which we obtain

$T[\mathrm{~K}]=12.5 /\left(\ln \left(R_{T}\right)+0.74\right.$.

Figure 25 (left) displays the observed dependence of $R_{T}$ on $R$. If the $\mathrm{SiO}$ layer is optically thin, the value of $R_{T}$ provides a direct measure of the temperature; if it is optically thick, the emission probes only the outer part of the $\mathrm{SiO}$ layer and measures a temperature that is representative of larger values of $r$. When $R$ increases, the observed value of $R_{T}$ remains nearly constant at $\sim 0.65$ up to $R \sim 1^{\prime \prime}$ and then increases to a value of $\sim 0.8$ at $1.5^{\prime \prime}$, corresponding to temperatures of $\sim 40 \mathrm{~K}$ and $\sim 24 \mathrm{~K}$, respectively. The value of $40 \mathrm{~K}$, associated with $R<1^{\prime \prime}$, is close to the lower value of the temperature obtained from the CO model for $r=1^{\prime \prime}$ $\left(T_{\mathrm{CO}}[\mathrm{K}]=70 \exp \left(-r / 2^{\prime \prime}\right)=42 \mathrm{~K}\right.$ for $\left.r=1^{\prime \prime}\right)$ in Sect. 4.1. This suggests that for low values of the projected distance from the star, $R$, the distance in space from the star, $r$, effectively probed by the $\mathrm{SiO}$ emission stays approximately constant and of the order of $1 "$. Indeed, the strong self-absorption causes the region probed by the $\mathrm{SiO}$ emission to be confined beyond $r \sim 1^{\prime \prime}$ (corresponding to $\tau_{\mathrm{SiO}} \sim 1$ ), but not to probe the volume beneath this surface. For larger values of the projected distance $R>1^{\prime \prime}$, we expect the distance in $3 \mathrm{D}$ space, $r$, of the effective $\mathrm{SiO}$-emitting surface to increase progressively as a result of the elongation of the emission volume along the line of poles, a pure geometrical effect (Fig. 20).

Qualitatively, we describe this trend in Fig. 25 (left) by assuming that $r$ stays at the $1^{\prime \prime}$ level for $R<1^{\prime \prime}$ and increases approximately from $1^{\prime \prime}$ to $2.5^{\prime \prime}$ when the projected $R$ increases from $1^{\prime \prime}$ to $2^{\prime \prime}$. Such a trend, when translated in terms of temperatures using the $\mathrm{CO}$ model relation $T_{\mathrm{CO}}[\mathrm{K}]=70 \exp \left(-r / 2^{\prime \prime}\right)$ gives a fair description of the observed dependence of $R_{T}$ on $R$ given the important approximations that have been made (Fig. 25 left).

The ratio $\varepsilon / \tau$ of the emissivity to optical depth is a measure of the lower limit of the emission of a self-absorbing layer. In the LTE approximation and to first order in $\Delta E / T$, where $\Delta E$ is the energy of the transition, $(\varepsilon / \tau) /\left(T v^{2}\right)$ is a constant, where $v$ is the frequency of the emission. Therefore, at the same temperature, $(\varepsilon / \tau)[\mathrm{SiO}(5-4)]=0.88(\varepsilon / \tau)\left[{ }^{12} \mathrm{CO}(2-1)\right]$. The value of $(\varepsilon / \tau)$ is $T / 26.3 \mathrm{Jy} \mathrm{arcsec}^{-2}$ for $\mathrm{CO}$ and $T / 29.5 \mathrm{Jy} \mathrm{arcsec}^{-2}$ for $\mathrm{SiO}$. Taking as reference $T=100 \mathrm{~K}$ for $\mathrm{SiO}$ and $50 \mathrm{~K}$ for $\mathrm{CO}$, the corresponding values of $(\varepsilon / \tau)$ are $1.9 \mathrm{Jy} \mathrm{arcsec}^{-2}$ for $\mathrm{CO}$ and $3.4 \mathrm{Jy} \mathrm{arcsec}^{-2}$ for $\mathrm{SiO}$. The observed values (Fig. 25 centerright) are $\sim 2.5$ and $6 \mathrm{Jy} \operatorname{arcsec}^{-2}$, respectively at $R \sim 0$. This is less than a factor 2 above the reference values, showing that the optical thickness is close to that of the self-absorption regime. A similar result has been observed for other AGB stars with massloss rates on the order of $10^{-7} M_{\odot} \mathrm{yr}^{-1}$ (R Dor, Nhung et al. 2021, W Hya, Takigawa et al. 2017).

To gain further insight into this issue, having obtained qualitative evidence for strong absorption, we need to properly account for radiative transfer. To do so, we use the model developed in Sect. 4.1 to describe the morpho-kinematics of the CO component of the CSE: in Fig. 26 we compare Doppler velocity spectra of $\mathrm{SiO}$ and $\mathrm{CO}$ in octants of position angle (the first one defined as $7^{\circ}<\omega<52^{\circ}$, then counter-clockwise for increasing numbers, as indicated on Fig. 23) in a ring $1^{\prime \prime}<R<1.5^{\prime \prime}$. This range of $R$ is far enough from the star to be relatively independent of modeling the star neighborhood, which contributes to the high-velocity wings of $\mathrm{SiO}$ spectra but our current data do not offer significant constraints on this region. We only show $\mathrm{SiO}(5-4)$ but the results are essentially the same for $\mathrm{SiO}(6-5)$. The $\mathrm{CO}$ data are seen to trace the polar outflows from the equatorial region, the $\mathrm{SiO}$ data do not. To adapt the $\mathrm{CO}$ model to a description of the $\mathrm{SiO}$ data, we keep all parameters as listed in Table 3 with the only exception being the radial profile: we divide the $\mathrm{CO}$ density parameter $\rho_{0}$ by three, and we further 

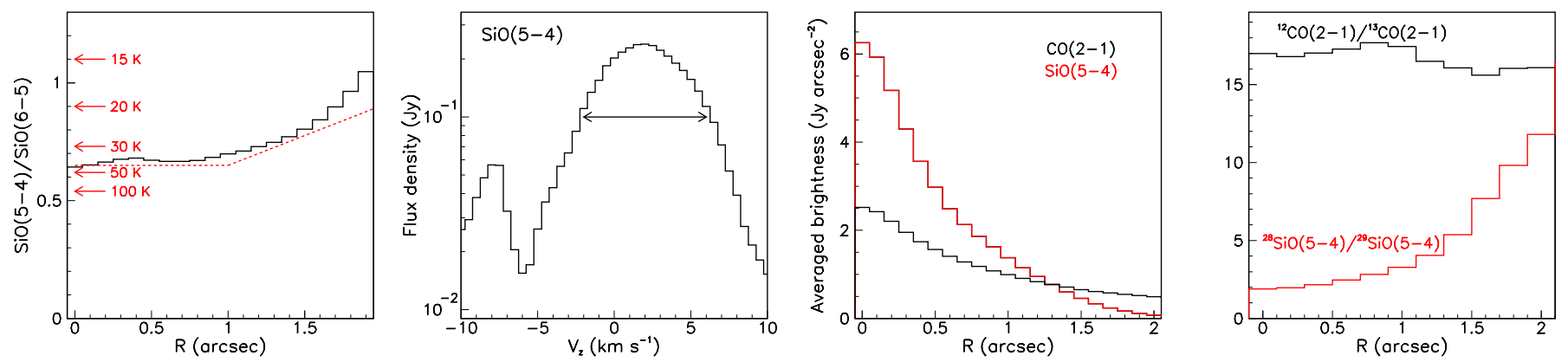

Fig. 25. Analysis of $\mathrm{SiO}$ and comparison with $\mathrm{CO}$. Left: ratio $R_{T}$ of $\mathrm{SiO}(5-4)$ to $\mathrm{SiO}(6-5)$ line emitted fluxes as a function of $R$ for $\left|v_{z}\right|<8 \mathrm{~km} \mathrm{~s}{ }^{-1}$. Corresponding temperature values using the relation $T[\mathrm{~K}]=12.5 /\left(\ln \left(R_{T}\right)+0.74\right.$ are shown in red on the scale of the ordinate. The red dashed line indicates constant $R_{T}$ of $0.64(T \sim 42 \mathrm{~K})$ for $R \leq 1^{\prime \prime}$ and a linear increase of $R_{T}$ to $0.89(T \sim 20 \mathrm{~K})$ from $1^{\prime \prime}$ to $2^{\prime \prime}$. Center-left: Doppler velocity spectrum of the $\mathrm{SiO}(5-4)$ data for $R<0.1^{\prime \prime}$. Center-right: $R$ distribution of the $\mathrm{SiO}(5-4)$ (red) and ${ }^{12} \mathrm{CO}(2-1)$ (black) emission averaged over position angle $\omega$, measured in Jy $\operatorname{arcsec}^{-2}$, and averaged over $-2<v_{z}<6 \mathrm{~km} \mathrm{~s}^{-1}$ as shown by the arrow in the center-left panel. Right: $R$ dependence of the ${ }^{12} \mathrm{CO}(2-1) /{ }^{13} \mathrm{CO}(2-1)$ ratio (black) and of the ${ }^{28} \mathrm{SiO}(5-4) / 29 \mathrm{SiO}(5-4)$ ratio (red).
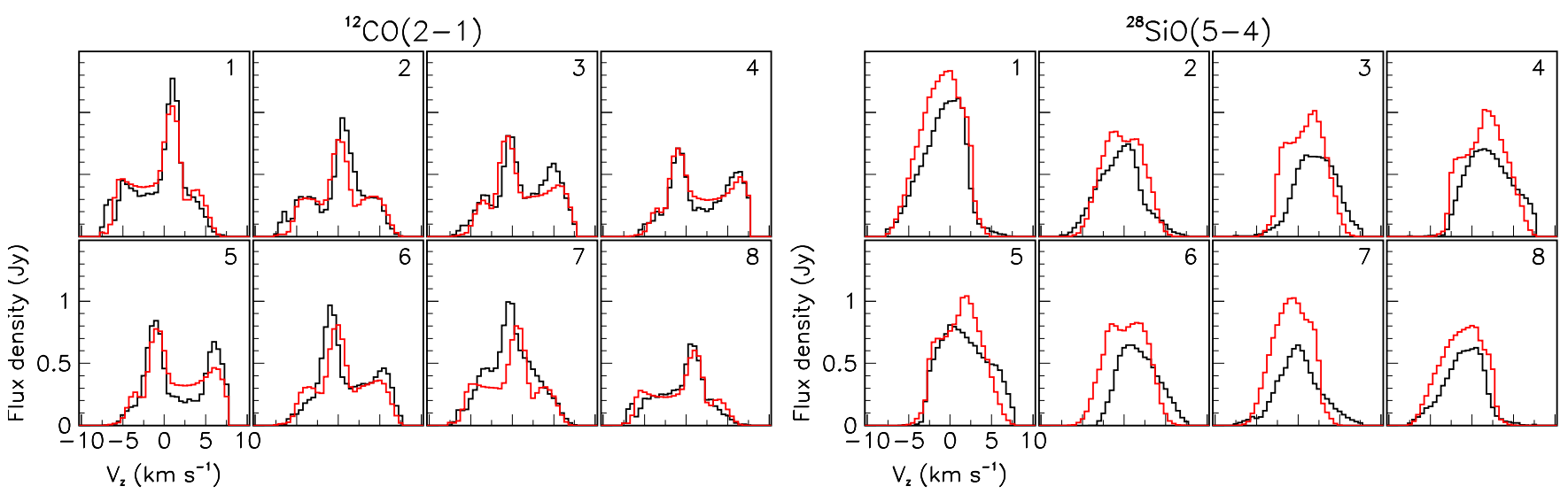

Fig. 26. ${ }^{12} \mathrm{CO}(2-1)$ and ${ }^{28} \mathrm{SiO}(5-4)$ Doppler velocity spectra in the ring $1^{\prime \prime}<R<1.5^{\prime \prime}$. The data are shown in black and the best-fit model results are shown in red (see text). Octant intervals in position angle $\omega$ are numbered as $1=\left[7^{\circ}, 52^{\circ}\right], 2=\left[52^{\circ}, 97^{\circ}\right], \ldots 8=\left[322^{\circ}, 7^{\circ}\right]$, these are indicated on the left and central panels of Fig. 23.

multiply it by $\exp (-r / 1.6)$, and set it to zero beyond $r=1.6^{\prime \prime}$, in qualitative accordance with the distributions displayed in the right panel of Fig. 23. These modifications result in a ratio of $\mathrm{SiO} / \mathrm{CO}=0.18$ at $r=1^{\prime \prime}$, in agreement with the estimate of Van de Sande et al. (2018a), who obtain a SiO/CO ratio of $0.17-0.19$ for this type of star.

Apart from this major modification of the radial distribution of the density, we use the same morpho-kinematics and same temperature distribution as for the best fit to the $\mathrm{CO}$ data. In order to adapt to $\mathrm{SiO}$, we change the parameters defining the transition, in particular with Einstein coefficients three orders of magnitude larger than for the $\mathrm{CO}$ transitions. The result, displayed in Fig. 26, describes the data surprisingly well given the crudeness of the exercise; in addition to offering an understanding of the large difference between $\mathrm{CO}$ and $\mathrm{SiO}$ emission, it provides confirmation of the validity of the description of the morpho-kinematics given by the model. What happens is that the $\mathrm{SiO}$ emission in the modeled annular ring between $1^{\prime \prime}$ and $1.5^{\prime \prime}$ probes only the outer layer of the (confined) $\mathrm{SiO}$ volume because of the very large absorption, while $\mathrm{CO}$ probes the lower density gas at much larger distances from the star where the equatorial region and the polar outflows are well separated. We refrain from attempting to adjust parameters to obtain a better fit: modeling the inner region could not be done reliably with the limited angular resolution of the present data.
Another illustration of the stronger absorption of the $\mathrm{SiO}$ line compared to the $\mathrm{CO}$ line is displayed in the right panel of Fig. 25. It compares the (projected) radial distribution of the ratio ${ }^{28} \mathrm{SiO}(5-4) /{ }^{29} \mathrm{SiO}(5-4)$ with that of the ratio ${ }^{12} \mathrm{CO}(2-1) /{ }^{13} \mathrm{CO}(2-$ 1). The ${ }^{29} \mathrm{SiO}$ data are obtained with a similar beam size to the ${ }^{28} \mathrm{SiO}$ and $\mathrm{CO}$ data, but with a spectral resolution of only $3 \mathrm{~km} \mathrm{~s}^{-1}$, preventing us from a detailed study of their Doppler velocity spectra. We expect the ${ }^{28} \mathrm{SiO} /{ }^{29} \mathrm{SiO}$ isotopic ratio to be $\sim 12$ or lower (De Beck \& Olofsson 2018, 2020), but this line ratio is reached only at large enough values of $R$, where the optical depth has decreased sufficiently for absorption to be less important. In the limit of complete self-absorption (i.e., emission at the $\varepsilon / \tau$ level), the line ratio is unity. In contrast, the $\mathrm{CO}$ ratio is much less affected by absorption, staying at $\sim 80 \%$ of the value evaluated in Sect. 4.1 ( $\sim 20)$ over the whole radial range.

\subsection{MHD wind models}

An important feature of the morpho-kinematics of RS Cnc is the latitudinal dependence of the velocity, which is larger towards the pole than along the equatorial plane. On the other hand, the modeling of Hoai et al. (2014) leads to an almost spheroidal distribution of matter (their Fig. 6), which implies an isotropic radiation field within the circumstellar shell (assuming position coupling between dust and gas). In these conditions, a driver 
other than radiation is needed to accelerate matter preferentially along the polar axis.

Axi-symmetric models have been developed on the assumption that a companion disturbs the gravitational field (Theuns \& Jorissen 1993; Mastrodemos \& Morris 1999; Decin et al. 2020). These models have been successful in explaining several observed features, such as the presence of spiral structures, although the preferential acceleration along the polar axis seems more difficult to reproduce.

In RS Cnc, we do not find evidence for the presence of a companion. While this negative result does not necessarily mean that there is no companion, we are considering here another mechanism that could play a role: the presence of a magnetic field. In this section, we argue that magnetic fields are one possible candidate to explain a significant pole-to-equator velocity contrast of $>1$.

Indeed, magnetic fields are an appealing way to explain some of the axi-symmetric shapes of planetary nebulae, such as polar jets or equatorial winds. Early simulations of winds with dipole fields and rotation showed that excess magnetic pressure could be able to repel the wind towards both the equator and/or the poles (Washimi \& Shibata 1993). Similar simulations also showed the formation of equatorial disks with enhanced outflow velocities in the equatorial region (Matt et al. 2000). In a more toroidal configuration, Matt \& Balick (2004) showed that both jets and equatorial disks could be produced depending on the magnitude of the rotation. Finally, García-Segura et al. (2005) showed that magnetically driven winds yield strongly anisotropic outflows with highly collimated polar jets.

Observational polarimetric studies (Greaves 2002) revealed ordered magnetic fields in planetary nebulae, with various degrees of toroidal configurations depending on the target. Using a handful of $\mathrm{SiO}$ masers (Herpin et al. 2006) or CN Zeeman measurements, Duthu et al. (2017) later attempted to further characterize the magnitude and the radial dependence of the magnetic field in the winds of AGB stars. These latter authors concluded that the field is on the order of a few Gauss near the stellar surface, and consistent with a $1 / r$ dependence on the distance $r$ from the star (see their Fig. 6).

In this section, we report results of simplistic calculations which integrate magnetized fluid parcel trajectories from the surface of an AGB star up to 10 stellar radii. We integrate the acceleration due to gravitational and Lorentz forces over time (pressure gradients become quickly negligible after the sonic point is crossed):

$\ddot{\boldsymbol{r}}=(\Gamma-1) \frac{G M_{*}}{r^{3}} \boldsymbol{r}+\frac{1}{\rho} \boldsymbol{J} \times \boldsymbol{B}$,

where $r$ is the position of the fluid parcel, $\Gamma$ is the ratio between the radiative and the gravitational force, $G$ is the universal gravity constant, $M_{*}$ is the stellar mass, and $\boldsymbol{J}=\frac{1}{4 \pi} \boldsymbol{\nabla} \times \boldsymbol{B}$ is the current vector. The mass density $\rho$ and the magnetic field $\boldsymbol{B}$ are prescribed, while we solve for the position and velocity of the fluid parcels. We parametrize our equations with $(\Gamma-1) G M_{*}=$ $v_{\infty} R_{*}^{2}$ and $\rho=\frac{\dot{M}}{4 \pi r^{2} \mathrm{v}_{\infty}}$. We assume $\dot{M}=1.24 \times 10^{-7} M_{\odot} \mathrm{yr}^{-1}$, and $R_{*}=1.6 \times 10^{13} \mathrm{~cm}$ as reasonable values for RS Cnc (Hoai et al. 2014). Our choice of parametrization allows us to easily explore nondimensional values of the parameters independently of the absolute observational constraints. After we obtain a suitable contrast between polar and equatorial velocities, we retrieve the physical value for the velocity scale - here $v_{\infty}=5.6 \mathrm{~km} \mathrm{~s}^{-1}-$ in order to obtain a given polar outflow velocity of $8 \mathrm{~km} \mathrm{~s}^{-1}$ at $r=10 R_{*}$. The initial velocity vector is set with a small uniform

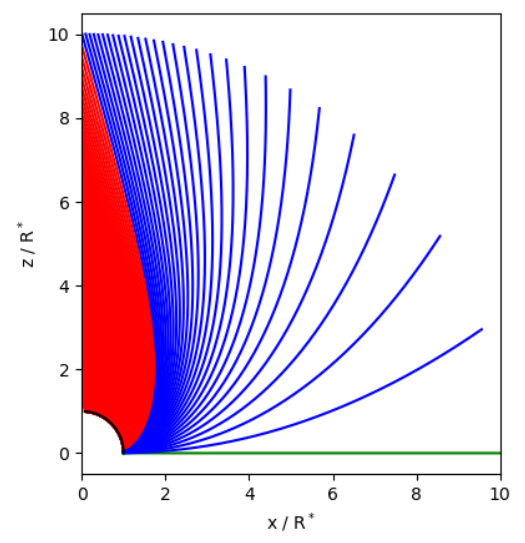

Fig. 27. Magnetized fluid parcel trajectories from a simplified model of RS Cnc (see text). Red trajectories meet on the polar axis, where they will likely generate a jet.

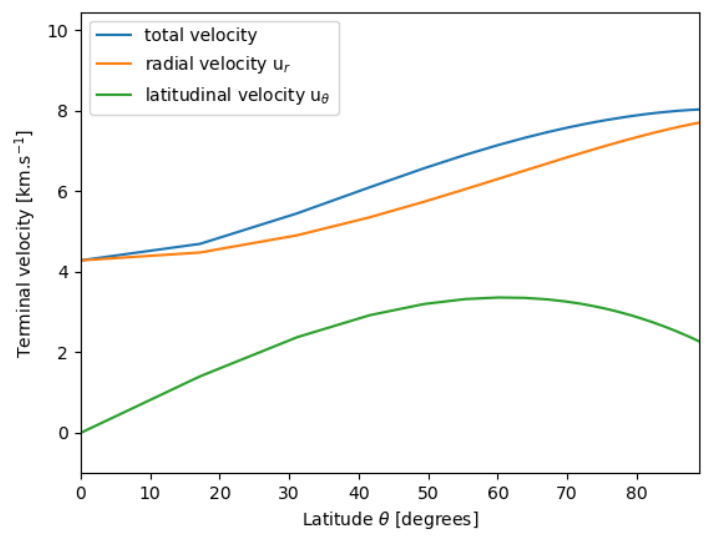

Fig. 28. Radial velocity at 10 stellar radii depending on the latitude for the same simplified model of magnetized wind as shown in Fig. 27.

radial velocity and we probe starting trajectories at the surface with a uniformly distributed initial latitude.

This simple setup allows us to quickly investigate various magnetic field configurations. Figure 27 displays the trajectories in the meridional plane obtained for a toroidal magnetic field with a $1 / r^{1.1}$ decline from $B_{*}=0.5 \mathrm{G}$ at the stellar surface: $B_{\phi}=B_{*} \cos (\theta) /\left(r / R_{*}\right)^{1.1}$ where $\theta$ is the latitude $\left(1 / r^{1.1}\right.$ gives a more pronounced velocity contrast between pole and equator than $1 / r$ ). The Lorentz force $\boldsymbol{J} \times \boldsymbol{B}$ in this case is directed toward the symmetry axis and acts as a focusing agent. All trajectories eventually end up on the axis where they would presumably launch a jet: this focuses mass loss towards the poles. Figure 28 shows the resulting "terminal" velocity at 10 stellar radii, where we have separated the latitudinal and the radial components. The flow velocities at this radius are dominated by their radial component, but with a clearly slower wind at the equator compared to the pole, as indicated by observations of, for instance, RS Cnc and EP Aqr. We note that rotation tends to produce the opposite effect (faster radial flow at equator compared to poles, due to centrifugal acceleration). We find similar behavior for toroidal magnetic field configurations closer to what Matt $\&$ Balick (2004) found $\left(B_{\phi}=3 B_{*} \cos (\theta) \sin (\theta)^{2} /\left(r / R_{*}\right)^{2}\right.$ with a $1 / r^{2}$ dependence and a concentration of $B$ at intermediate latitudes). We investigated additional dipolar fields which are able to generate some amount of rotation in the wind. Thanks to the versatility of the present setting, we can quickly explore various 
configurations, linear combinations between them, several magnetic field decay exponents, but have not yet investigated them systematically. The purpose of our investigation here is simply to show that a magnetic field is a valid candidate to produce poleto-equator velocity ratios of significantly greater than 1 . Finally, we note that our termination radius of $r=10 R_{*}$ is arbitrary, and a pertinent match to the observations could be considered at various distances depending on where the given tracer is expected to be concentrated. These crude models are still far from quantitatively matching the observational constraints from RS Cnc or EP Aqr, which require a broader polar outflow and a thinner and denser equatorial disk. This could be adjusted by providing a sharper toroidal magnetic barrier to funnel the wind at the appropriate places. However, such fine-tuning would overcome the limits of this crude exercise which still lacks self-consistency as the density profile remains radial and unaffected by the magnetic constraints (themselves blind to the wind), and shocks generated by the crossings of trajectories at the polar axis are not accounted for. We plan to investigate further with such simplified models in future work as they might provide a useful means to constrain the magnetic field configuration from the morphokinetics.

\subsection{HCN in M- to S-type stars}

Cherchneff (2006) recognized that the formation of $\mathrm{CN} / \mathrm{HCN}$ depends on the high activation barrier of the $\mathrm{H}+\mathrm{C}_{2} \rightarrow \mathrm{CH}+\mathrm{C}$ reaction, followed by rapid $\mathrm{CN}$ formation via $\mathrm{N}+\mathrm{CH} \rightarrow \mathrm{CN}+\mathrm{H}$. Their abundance therefore depends on thermal excursions in shocks, or inhomogeneities of temperature. In addition, both the total rate of formation of the pair $\mathrm{CN} / \mathrm{HCN}$ and the respective share between $\mathrm{CN}$ and $\mathrm{HCN}$ depend on the $\mathrm{H} / \mathrm{H}_{2}$ ratio which itself depends on out-of-equilibrium chemistry because of the slow conversion between $\mathrm{H}$ and $\mathrm{H}_{2}$. Cherchneff (2006) was thus able to show that the shocks produced by the pulsations close to the stellar photosphere could produce highly increased yields of the $\mathrm{HCN}$ molecule in $\mathrm{M}$ or S-type stars, despite their high $\mathrm{O} / \mathrm{C}$ ratio. We note here that magnetic fields produce shocks on the symmetry axis which might also help to boost HCN production in the polar jet.

HCN has long been detected and surveyed in M-type and S-type stars (e.g., Deguchi \& Goldsmith 1985; Lindqvist et al. 1988; Bujarrabal et al. 1994; Olofsson et al. 1998; Schöier et al. 2013). RS Cnc on the other hand has never been detected in ground-state or vibrationally excited $\mathrm{HCN}$ despite various observational efforts with different telescopes (Lucas et al. 1988; Sopka et al. 1989; Nercessian et al. 1989; Lindqvist et al. 1992; Bujarrabal et al. 1994; Bieging \& Latter 1994; Olofsson et al. 1998). Adopting a mass-loss rate of $3 \times 10^{-7} M_{\odot} \mathrm{yr}^{-1}$, Bujarrabal et al. (1994) estimated an upper limit to the $\mathrm{HCN}$ abundance in RS Cnc of $4.5 \times 10^{-7}$. This upper limit becomes $1.35 \times 10^{-6}$ if we adopt $\dot{M}=1 \times 10^{-7} M_{\odot} \mathrm{yr}^{-1}$. Schöier et al. (2013) presented a comprehensive analysis of the HCN abundance in a sample of 59 AGB stars, including 25 carbon-rich, 19 S-type, and 25 M-type stars, by means of a non-LTE radiative transfer modeling. For M-type and S-type stars, these authors derived a median $\mathrm{HCN} / \mathrm{H}_{2}$ abundance of order $1 \times 10^{-7}$ and $7 \times 10^{-7}$, respectively, with a large spread between $\sim 5 \times 10^{-8}$ and $\sim 5 \times 10^{-6}$.

By an XCLASS modeling of the HCN(3-2) line detected here (see Appendix D), and assuming a rotational temperature of $350 \mathrm{~K}$, we derive an $\mathrm{HCN}$ column density of $\sim 1.6 \times 10^{15} \mathrm{~cm}^{-2}$; see Fig. 29. With the same assumptions as those made in Sect. 3.6, this translates to $\mathrm{HCN}$ abundances of $X(\mathrm{HCN} /\langle\mathrm{H}\rangle)=$

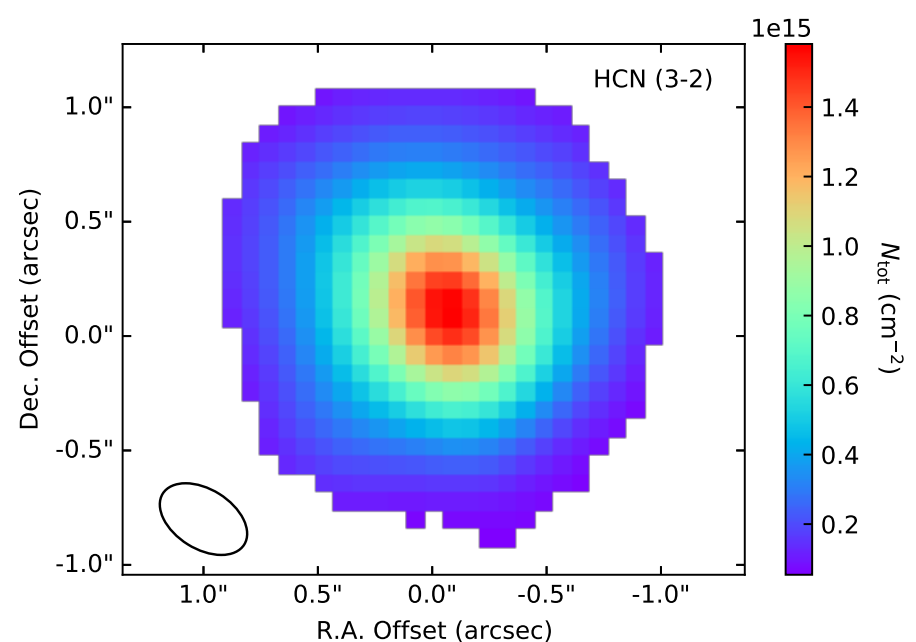

Fig. 29. Map of the HCN column density as derived by an XCLASS modeling (see Appendix D). The black ellipse in the lower left corner indicates the synthesized beam.

$3.3 \times 10^{-7}$, or, if hydrogen were completely bound in $\mathrm{H}_{2}$, $X\left(\mathrm{HCN} / \mathrm{H}_{2}\right)=6.6 \times 10^{-7}$. This abundance perfectly fits in the range found by Schöier et al. (2013) for M- to S-type stars and is also consistent with the upper limit derived by Bujarrabal et al. (1994) corrected for the mass-loss rate.

Clumpy and porous winds help UV photons to penetrate closer to the star, thus photodissociating $\mathrm{CO}$ and $\mathrm{N}_{2}$ to release more of the C/NO and the N/CS pairs of reactants, which both produce $\mathrm{CN}$ : this process was shown to considerably enhance the HCN abundances close to the star (Van de Sande et al. 2018b, 2020). Rather than uniformly distributed random clumpiness and porosity, one can also imagine ordered density distributions in the wind, which could let UV photons penetrate through lowdensity channels. These structures could sometimes be hard to witness due to line of sight confusion. In fact, our simplified magnetized wind models (see Fig. 27 with scarcity of trajectories around the equator) or more sophisticated magnetized wind models (e.g., Matt et al. 2000; Washimi \& Shibata 1993) allow for lower column-density channels at certain angles. These may result in increased HCN abundance, but chemical postprocessing in magnetized models will be necessary to assess whether this is a viable interpretation of the observations.

\section{Conclusions}

Using NOEMA equipped with PolyFiX, we obtained highspatial resolution $\left(\sim 0.3^{\prime \prime}\right)$ images of $\mathrm{RS}$ Cnc in several lines of different molecules. We detect, and in most cases are able to map, 32 lines of 13 molecules and isotopologs $\left(\mathrm{CO},{ }^{13} \mathrm{CO}\right.$, $\left.\mathrm{SiO},{ }^{29} \mathrm{SiO}, \mathrm{SO},{ }^{34} \mathrm{SO}, \mathrm{SO}_{2}, \mathrm{H}_{2} \mathrm{O}, \mathrm{HCN}, \mathrm{H}^{13} \mathrm{CN}, \mathrm{PN}\right)$, including several transitions from vibrationally excited states, and a tentative identification of $\mathrm{Si}^{17} \mathrm{O}$ and possibly ${ }^{29} \mathrm{Si}^{17} \mathrm{O}$. HCN as well as millimeter vibrationally excited $\mathrm{H}_{2} \mathrm{O}, \mathrm{SO}, \mathrm{SO}_{2}$, and $\mathrm{PN}$ and their isotopologs are first detections in RS Cnc. From their first-moment maps, some of the lines, $\mathrm{SiO}(v=1,6-5), \mathrm{HCN}$, $\mathrm{SO}, \mathrm{SO}_{2}$, show signs of rotation in the close vicinity of the star. A population diagram analysis for the 11 observed $\mathrm{SO}_{2}$ lines provides a rotational temperature of about $320 \mathrm{~K}$ in the region that shows signs of rotation. Temperatures of this order are also found from an XCLASS modeling of the $\mathrm{SO}_{2}$ lines. For $\mathrm{SO}_{2}$ and $\mathrm{HCN}$, we find column densities from the XCLASS modeling of $N_{\mathrm{SO}_{2}} \sim 3.5 \times 10^{15} \mathrm{~cm}^{-2}$ and $N_{\mathrm{HCN}} \sim 1.6 \times 10^{15} \mathrm{~cm}^{-2}$, 
which translate to abundance ratios of $X\left(\mathrm{SO}_{2} / \mathrm{H}_{2}\right)=1.5 \times 10^{-6}$ and $X\left(\mathrm{HCN} / \mathrm{H}_{2}\right)=6.6 \times 10^{-7}$, respectively, well within the range expected for an MS-type star.

We find broad wings in the spectral line profiles of vibrational ground-state transitions of $\mathrm{SiO}$ and $\mathrm{SO}$ and in first vibrationally excited transitions of $\mathrm{SiO}$, which indicate radial velocities of about twice the terminal outflow velocity as probed by $\mathrm{CO}$. As high velocities very close to the star are also seen in similar objects, such as EP Aqr, $o$ Cet, and R Dor, the presence of these broad line wings calls for a mechanism common to the class of pulsating AGB stars. We interpret these high-velocity line wings as the imprints of pulsation shocks acting in the very inner region around these stars.

The spatially resolved images allow us to trace the morphokinematics of the wind around RS Cnc at different scales. In the inner part $\left(<0.5^{\prime \prime}\right.$, or $\left.75 \mathrm{AU}\right)$, we find a rotating structure well traced by the less abundant molecules $\left(\mathrm{HCN}, \mathrm{SO}, \mathrm{SO}_{2}\right)$, and by $\mathrm{SiO}$ in $(v=1)$ lines. Outside $75 \mathrm{AU}$, we find an expanding axisymmetric outflow, with velocities $\sim 4 \mathrm{~km} \mathrm{~s}^{-1}$ in the equatorial plane, and $\sim 9 \mathrm{~km} \mathrm{~s}^{-1}$ along the polar axis. This polar axis coincides with the axis of the internal rotating structure. A model that fits the data cubes obtained on the ${ }^{12} \mathrm{CO}(2-1),{ }^{13} \mathrm{CO}(2-1)$ and $\mathrm{SiO}(v=0,5-4$ and 6-5) lines gives a mass-loss rate of $1 \times 10^{-7} M_{\odot} \mathrm{yr}^{-1}$ for the equatorial region (latitude $<30^{\circ}$ ) and of $2 \times 10^{-7} M_{\odot} \mathrm{yr}^{-1}$ for the polar outflows (latitude $>30^{\circ}$ ). The ${ }^{12} \mathrm{CO} /{ }^{13} \mathrm{CO}$ ratio is measured to be $\sim 20$ on average, $24 \pm 2$ in the polar outflows and $19 \pm 3$ in the equatorial region.

Although we cannot exclude the possibility that an unseen stellar or substellar companion shapes the circumstellar environment of RS Cnc, we also consider the possibility of a magnetic field playing this role. In particular, a toroidal magnetic field configuration would provide a mechanism able to produce the significant velocity contrast between high polar-outflow velocities and low expansion velocities in the equatorial region that is observed in RS Cnc and other similar stars.

Acknowledgements. We thank the staff at the NOEMA and Pico Veleta observatories for their support of these observations. The authors are grateful to the anonymous referee for a very detailed and valuable report that helped improving the presentation of the material. This work is based on observations carried out under project numbers W16BE, D17AE, W19AX with the IRAM NOEMA interferometer and under project ID 136-19 with the IRAM 30m telescope. IRAM is supported by INSU/CNRS (France), MPG (Germany) and IGN (Spain). The Ha Noi team acknowledges financial support from the World Laboratory, the Odon Vallet Foundation and VNSC. This research is funded in part by the Vietnam National Foundation for Science and Technology Development (NAFOSTED) under grant number 103.99-2019.368. This work was supported by the Programme National "Physique et Chimie du Milieu Interstellaire" (PCMI) of CNRS/INSU with INC/INP co-funded by CEA and CNES. This work has made use of data from the European Space Agency (ESA) mission Gaia (https: //www . cosmos.esa.int/gaia), processed by the Gaia Data Processing and Analysis Consortium (DPAC, https://www. cosmos.esa.int/web/ gaia/dpac/consortium). Funding for the DPAC has been provided by national institutions, in particular the institutions participating in the Gaia Multilateral Agreement.

\section{References}

Adelman, S. J., \& Dennis, J. W., III. 2005, Balt. Astron., 14, 41

Alcolea, J., \& Menten, K. M. 1993, The Excitation of Vibrationally Excited $\mathrm{H}_{2} \mathrm{O}$ Masers. Astrophysical Masers, 412, eds. A. W. Clegg, \& G. E. Nedoluha, 399

Bailer-Jones, C. A. L., Rybizki, J., Fouesneau, M., Demleitner, M., \& Andrae, R. 2021, VizieR Online Data Catalog: I/352

Belov, S. P., Kozin, I. N., Polyansky, O. L., Tret'yakov, M. Y., \& Zobov, N. F. 1987, J. Mol. Spectr., 126, 113

Bieging, J. H., \& Latter, W. B. 1994, ApJ, 422, 765

Bujarrabal, V., Fuente, A., \& Omont, A. 1994, A\&A, 285, 247

Cernicharo, J., Guélin, M., \& Kahane, C. 2000, A\&AS, 142, 181

Cherchneff, I. 2006, A\&A, 456, 1001
Chin, Y. N., Henkel, C., Whiteoak, J. B., Langer, N., \& Churchwell, E. B. 1996, A\&A, 305, 960

Cristallo, S., Piersanti, L., Straniero, O., et al. 2011, ApJS, 197, 17

Danilovich, T., Teyssier, D., Justtanont, K., et al. 2015, A\&A, 581, A60

Danilovich, T., De Beck, E., Black, J. H., Olofsson, H., \& Justtanont, K. 2016, A\&A, 588, A119

Danilovich, T., Richards, A. M. S., Decin, L., Van de Sande, M., \& Gottlieb, C. A. 2020, MNRAS, 494, 1323

De Beck, E., \& Olofsson, H. 2018, A\&A, 615, A8

De Beck, E., \& Olofsson, H. 2020, A\&A, 642, A20

De Beck, E., Kamiński, T., Patel, N. A., et al. 2013, A\&A, 558, A132

De Nutte, R., Decin, L., Olofsson, H., et al. 2017, A\&A, 600, A71

de Vicente, P., Bujarrabal, V., Díaz-Pulido, A., et al. 2016, A\&A, 589, A74

Decin, L., Cherchneff, I., Hony, S., et al. 2008, A\&A, 480, 431

Decin, L., Richards, A. M. S., Danilovich, T., Homan, W., \& Nuth, J. A. 2018 , A\&A, 615, A28

Decin, L., Montargès, M., Richards, A. M. S., et al. 2020, Science, 369, 1497

Deguchi, S., \& Goldsmith, P. F. 1985, Nature, 317, 336

Dickinson, D. F., Bechis, K. P., \& Barrett, A. H. 1973, ApJ, 180, 831

Doan, L., Ramstedt, S., Vlemmings, W. H. T., et al. 2017, A\&A, 605, A28

Dorfi, E., \& Höfner, S. 1996, A\&A, 313, 605

Dumm, T., \& Schild, H. 1998, New Astron., 3, 137

Duthu, A., Herpin, F., Wiesemeyer, H., et al. 2017, A\&A, 604, A12

Endres, C. P., Schlemmer, S., Schilke, P., Stutzki, J., \& Müller, H. S. P. 2016, J. Mol. Spectr., 327, 95

Feast, M. W. 1953, MNRAS, 113, 510

Freytag, B., Liljegren, S., \& Höfner, S. 2017, A\&A, 600, A137

Gaia Collaboration (Brown, A. G. A., et al.) 2021, A\&A, 649, A1

García-Segura, G., López, J. A., \& Franco, J. 2005, ApJ, 618, 919

Gérard, E., \& Le Bertre, T. 2003, A\&A, 397, L17

Glanz, H., \& Perets, H. B. 2018, MNRAS, 478, L12

Goldsmith, P. F., \& Langer, W. D. 1999, ApJ, 517, 209

Gottlieb, C. A., Decin, L., Richards, A. M. S., et al. 2022, A\&A, in press, https : //doi.org/10.1051/0004-6361/202140431

Gray, M. D., Baudry, A., Richards, A. M. S., et al. 2016, MNRAS, 456, 374

Greaves, J. S. 2002, A\&A, 392, L1

Guélin, M., Muller, S., Cernicharo, J., et al. 2000, A\&A, 363, L9

Han, F., Mao, R. Q., Lei, C. M., et al. 1995, Publ. Purple Mountain Observ., 14, 185

Herpin, F., Baudry, A., Thum, C., Morris, D., \& Wiesemeyer, H. 2006, A\&A, 450,667

Hinkle, K. H., Hall, D. N. B., \& Ridgway, S. T. 1982, ApJ, 252, 697

Hinkle, K. H., Lebzelter, T., \& Straniero, O. 2016, ApJ, 825, 38

Hoai, D. T., Matthews, L. D., Winters, J. M., et al. 2014, A\&A, 565, A54

Hoai, D. T., Nhung, P. T., Tuan-Anh, P., et al. 2019, MNRAS, 484, 1865

Hoai, D. T., Tuan-Anh, P., Nhung, P. T., et al. 2020, MNRAS, 495, 943

Höfner, S., \& Olofsson, H. 2018, A\&ARv, 26, 1

Högbom, J. A. 1974, A\&AS, 15, 417

Homan, W., Danilovich, T., Decin, L., et al. 2018a, A\&A, 614, A113

Homan, W., Richards, A., Decin, L., de Koter, A., \& Kervella, P. 2018b, A\&A, 616, A34

Homan, W., Montargès, M., Pimpanuwat, B., et al. 2020, A\&A, 644, A61

Hughes, G. L., Gibson, B. K., Carigi, L., et al. 2008, MNRAS, 390, 1710

Humire, P. K., Thiel, V., Henkel, C., et al. 2020, A\&A, 642, A222

Joyce, R. R., Hinkle, K. H., Wallace, L., Dulick, M., \& Lambert, D. L. 1998, AJ, 116,2520

Kamiński, T., Gottlieb, C. A., Young, K. H., Menten, K. M., \& Patel, N. A. 2013, ApJS, 209, 38

Karakas, A. I., \& Lattanzio, J. C. 2014, Publ. Astron. Soc. Aust., 31, e030

Keenan, P. C. 1954, ApJ, 120, 484

Khouri, T., Vlemmings, W. H. T., Olofsson, H., et al. 2018, A\&A, 620, A75

Le Bertre, T., Hoai, D. T., Nhung, P. T., \& Winters, J. M. 2016, in SF2A-2016: Proceedings of the Annual meeting of the French Society of Astronomy and Astrophysics, eds. C. Reylé, J. Richard, L. Cambrésy, et al., 433

Lebzelter, T., \& Hron, J. 1999, A\&A, 351, 533

Lewis, B. M. 1997, AJ, 114, 1602

Libert, Y., Winters, J. M., Le Bertre, T., Gérard, E., \& Matthews, L. D. 2010, A\&A, 515, A112

Lindqvist, M., Nyman, L.-Å., Olofsson, H., \& Winnberg, A. 1988, A\&A, 205, L15

Lindqvist, M., Olofsson, H., Winnberg, A., \& Nyman, L. A. 1992, A\&A, 263, 183

Lodders, K., Palme, H., \& Gail, H.-P. 2009, 4.4 Abundances of the elements in the Solar System: Datasheet from Landolt-Börnstein - Group VI Astronomy and Astrophysics · Volume 4B: "Solar System" in Springer Materials (https: //doi .org/10.1007/978-3-540-88055-4_34)

Lovas, F. J. 1985, J. Phys. Chem. Ref. Data, 14, 395

Lucas, R., Guilloteau, S., \& Omont, A. 1988, A\&A, 194, 230 
Mamon, G. A., Glassgold, A. E., \& Huggins, P. J. 1988, ApJ, 328, 797

Mastrodemos, N., \& Morris, M. 1999, ApJ, 523, 357

Matt, S., \& Balick, B. 2004, ApJ, 615, 921

Matt, S., Balick, B., Winglee, R., \& Goodson, A. 2000, ApJ, 545, 965

Matthews, L. D., \& Reid, M. J. 2007, AJ, 133, 2291

Menten, K. M., \& Melnick, G. J. 1989, ApJ, 341, L91

Menten, K. M., Philipp, S. D., Güsten, R., et al. 2006, A\&A, 454, L107

Merrill, K. M., \& Stein, W. A. 1976, PASP, 88, 285

Milam, S. N., Halfen, D. T., Tenenbaum, E. D., et al. 2008, ApJ, 684, 618

Möller, T., Endres, C., \& Schilke, P. 2017, A\&A, 598, A7

Montez, Rodolfo, J., Ramstedt, S., Kastner, J. H., Vlemmings, W., \& Sanchez, E. 2017, ApJ, 841, 33

Müller, H. S. P., \& Brünken, S. 2005, J. Mol. Spectro., 232, 213

Müller, H. S. P., Schlöder, F., Stutzki, J., \& Winnewisser, G. 2005, J. Mol. Struct. 742,215

Müller, H. S. P., Thorwirth, S., Roth, D. A., \& Winnewisser, G. 2001, A\&A, 370, L49

Müller, H. S. P., Spezzano, S., Bizzocchi, L., et al. 2013, J. Phys. Chem. A, 117, 13843

Nercessian, E., Guilloteau, S., Omont, A., \& Benayoun, J. J. 1989, A\&A, 210, 225

Nhung, P. T., Hoai, D. T., Winters, J. M., et al. 2015a, Res. Astron. Astrophys., 15,713

Nhung, P. T., Hoai, D. T., Winters, J. M., et al. 2015b, A\&A, 583, A64

Nhung, P. T., Hoai, D. T., Tuan-Anh, P., et al. 2018, MNRAS, 480, 3324

Nhung, P. T., Hoai, D. T., Tuan-Anh, P., et al. 2019a, MNRAS, 490, 3329

Nhung, P. T., Hoai, D. T., Tuan-Anh, P., et al. 2019b, Res. Astron. Astrophys., 19, 043

Nhung, P. T., Hoai, D. T., Tuan-Anh, P., et al. 2021, MNRAS, 504, 2687

Noguchi, K., \& Kobayashi, Y. 1993, PASJ, 45, 85

Nomoto, K., Thielemann, F. K., \& Yokoi, K. 1984, ApJ, 286, 644

Norris, B. R. M., Tuthill, P. G., Ireland , M. J., et al. 2012, Nature, 484, 220

Nowotny, W., Lebzelter, T., Hron, J., \& Höfner, S. 2005, A\&A, 437, 285

Nyman, L.-A.., Booth, R. S., Carlström, U., et al. 1992, A\&AS, 93, 121

Ohnaka, K., Weigelt, G., \& Hofmann, K.-H. 2019, ApJ, 883, 89

Olofsson, H., Lindqvist, M., Nyman, L.-Å.., \& Winnberg, A. 1998, A\&A, 329, 1059

Olofsson, H., Vlemmings, W. H. T., Maercker, M., et al. 2015, A\&A, 576, L15

Omont, A., Lucas, R., Morris, M., \& Guilloteau, S. 1993, A\&A, 267, 490

Pardo, J. R., Alcolea, J., Bujarrabal, V., et al. 2004, A\&A, 424, 145

Pearson, J. C., Anderson, T., Herbst, E., De Lucia, F. C., \& Helminger, P. 1991, ApJ, 379, L41

Pickett, H. M., Poynter, R. L., Cohen, E. A., et al. 1998, J. Quant. Spectr. Rad. Transf., 60, 883
Richter, H., Wood, P. R., Woitke, P., Bolick, U., \& Sedlmayr, E. 2003, A\&A, 400, 319

Rizzo, J. R., Cernicharo, J., \& García-Miró, C. 2021, ApJS, 253, 44

Sahai, R. 1992, A\&A, 253, L33

Schöier, F. L., Olofsson, H., Wong, T., Lindqvist, M., \& Kerschbaum, F. 2004, A\&A, 422, 651

Schöier, F. L., Ramstedt, S., Olofsson, H., et al. 2013, A\&A, 550, A78

Smith, V. V., \& Lambert, D. L. 1986, ApJ, 311, 843

Smith, V. V., \& Lambert, D. L. 1990, ApJS, 72, 387

Sopka, R. J., Olofsson, H., Johansson, L. E. B., Nguyen-Q-Rieu, \& Zuckerman, B. 1989, A\&A, 210, 78

Spinrad, H., Pyper, D. M., Newburn, Ray L., J., \& Younkin, R. L. 1966, ApJ, 143,291

Stancliffe, R. J., Tout, C. A., \& Pols, O. R. 2004, MNRAS, 352, 984

Stephenson, C. B. 1984, Publ. Warner Swasey Observ., 3, 1

Szymczak, M., \& Engels, D. 1995, A\&A, 296, 727

Takigawa, A., Kamizuka, T., Tachibana, S., \& Yamamura, I. 2017, Sci. Adv., 3, eaao 2149

Theuns, T., \& Jorissen, A. 1993, MNRAS, 265, 946

Timmes, F. X., Woosley, S. E., \& Weaver, T. A. 1995, ApJS, 98, 617

Tuan-Anh, P., Hoai, D. T., Nhung, P. T., et al. 2019, MNRAS, 487, 622

Van de Sande, M., Decin, L., Lombaert, R., et al. 2018a, A\&A, 609, A63

Van de Sande, M., Sundqvist, J. O., Millar, T. J., et al. 2018b, A\&A, 616, A106

Van de Sande, M., Sundqvist, J. O., Millar, T. J., et al. 2020, A\&A, 634, C1

Velilla Prieto, L., Sánchez Contreras, C., Cernicharo, J., et al. 2017, A\&A, 597, A25

Vlemmings, W., Khouri, T., O'Gorman, E., et al. 2017, Nat. Astron., 1, 848

Vlemmings, W. H. T., Khouri, T., De Beck, E., et al. 2018, A\&A, 613, L4

Washimi, H., \& Shibata, S. 1993, MNRAS, 262, 936

Wilson, T. L., \& Matteucci, F. 1992, A\&ARv, 4, 1

Winters, J. M., Keady, J. J., Gauger, A., \& Sada, P. V. 2000a, A\&A, 359, 651

Winters, J. M., Le Bertre, T., Jeong, K. S., Helling, C., \& Sedlmayr, E. 2000b, A\&A, 361, 641

Winters, J. M., Le Bertre, T., Nyman, L.-^̊., Omont, A., \& Jeong, K. S. 2002, A\&A, 388, 609

Winters, J. M., Le Bertre, T., Jeong, K. S., Nyman, L.-Å., \& Epchtein, N. 2003, A\&A, 409, 715

Winters, J. M., Le Bertre, T., Pety, J., \& Neri, R. 2007, A\&A, 475, 559 (W2007)

Wong, K. T., Kamiński, T., Menten, K. M., \& Wyrowski, F. 2016, A\&A, 590, A127

Yoon, D.-H., Cho, S.-H., Kim, J., Yun, Y. j., \& Park, Y.-S. 2014, ApJS, 211, 15

Yu, S., Pearson, J. C., Drouin, B. J., et al. 2012, J. Mol. Spectr., 279, 16

Ziurys, L. M., Schmidt, D. R., \& Bernal, J. J. 2018, ApJ, 856, 169 


\section{Appendix A: Resolved out flux}

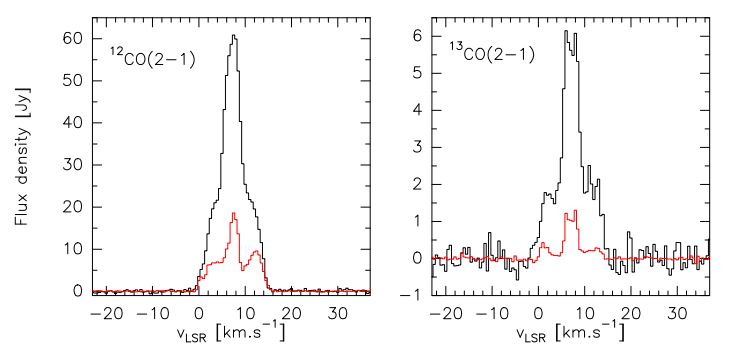

Fig. A.1. Effect of missing short spacing information on the $\mathrm{CO}$ lines. Left: ${ }^{12} \mathrm{CO}(2-1)$. Right: ${ }^{13} \mathrm{CO}(2-1)$. A-configuration and Dconfiguration are merged, the spectral resolution is $0.5 \mathrm{~km} \mathrm{~s}^{-1}$ and the $\mathrm{CO}$ emission is integrated over the central $22^{\prime \prime} \times 22^{\prime \prime}$, i.e., over the full field of view of the NOEMA antennas at $230 \mathrm{GHz}$. Black profiles: OTF data (i.e., the short-spacing information that is filtered out by the interferometer) are added. Red profiles: A+D configuration interferometer data, only.

The effect on the flux of filtering out large-scale structure with the interferometer is shown in Fig. A.1 for the $\mathrm{CO}$ and ${ }^{13} \mathrm{CO}$ lines. These two are the only lines discussed in this paper that are affected by the short-spacing problem.

\section{Appendix B: Intensity maps}

Here we present velocity-integrated intensity maps in three velocity ranges for $\mathrm{HCN}$ and $\mathrm{H}^{13} \mathrm{CN}$ (Fig. B.1), the four detected $\mathrm{SO}$ lines (Fig. B.3), and three out of the $11 \mathrm{SO}_{2}$ lines (Fig. B.4). These are the $\mathrm{SO}_{2}$ line with the lowest upper level energy, the strongest $\mathrm{SO}_{2}$ line detected here, and the $\mathrm{SO}_{2}$ line with the highest upper level energy, respectively. All these nine lines display kinematic structure in east-west direction. Also shown are zeroth moment maps for the (unresolved) $\mathrm{H}_{2} \mathrm{O}$ lines (Fig. B.2) and for the (weak) PN line (Fig. B.5).

\section{Appendix C: $\mathrm{SO}_{2}$ line profiles}

In this section, we present the line profiles of all the $11 \mathrm{SO}_{2}$ lines detected with our setups (see Fig. C.1).

\section{Appendix D: XCLASS modeling of $\mathrm{HCN}$ and $\mathrm{SO}_{2}$}

The $\mathrm{HCN}(3-2)$ line and the 11 detected $\mathrm{SO}_{2}$ emission lines were modeled using the eXtended CASA Line Analysis Software Suite (XCLASS ${ }^{7}$, Möller et al. 2017). XCLASS models and fits molecular lines by solving the $1 \mathrm{D}$ radiative transfer equation with the assumption of LTE and of an isothermal source. Here, 1D means that the radiative transfer equation is integrated along the line of sight. Spectral lines are fitted with Gaussian profiles, and optical depth effects and source size are considered in the calculations. Molecular properties (e.g., Einstein coefficients, partition functions, etc.) are taken from an embedded SQLite database containing entries from the Cologne Database for Molecular Spectroscopy (CDMS, Müller et al. 2001, 2005) and from the Jet Propulsion Laboratory database (JPL, Pickett et al. (1998)) using the Virtual Atomic and Molecular Data Center (VAMDC, Endres et al. 2016). The fit parameter set for each line component consists of the source size $\theta_{\text {source }}$, the rotation temperature $T_{\text {rot }}$, the total column density $N_{\text {tot }}$, the line width $\Delta \mathrm{v}$, and the velocity offset $\mathrm{v}_{\text {off }}$ (given here in the LSR system).

The XCLASS package offers various algorithms to find the best-fit parameters by minimizing the $\chi^{2}$ value, and here we utilized the Levenberg-Marquardt (LM) method. To obtain maps of the physical parameters, we use the myXCLASSMapFIt function to fit $\mathrm{HCN}(3-2)$ and the 11 detected $\mathrm{SO}_{2}$ emission lines (see Fig. C.1) pixel by pixel.

For $\mathrm{SO}_{2}$, we modeled and fitted 11 lines simultaneously with a threshold of $18 \sigma$ and a single Gaussian component. All fit parameters are regarded as free parameters in the fitting process. The XCLASS models for $\mathrm{SO}_{2}$ result in a temperature of $T_{\text {rot }} \sim$ $350 \mathrm{~K}$ (Fig. D.1), somewhat higher than the result from our population diagram analysis, but within the error bars (see Sect. 3.6). It also results in an average line optical depth of 0.1 , confirming that the assumption of the lines being optically thin is justified when constructing the population diagram. Assuming LTE, the derived rotation temperature equals the kinetic gas temperature $T_{\text {kin }}$. For the $\mathrm{SO}_{2}$ column density, the XCLASS modeling results in a value of $N_{\mathrm{SO}_{2}} \sim 3.5 \times 10^{15} \mathrm{~cm}^{-2}$, almost identical to the result from the population diagram analysis presented in Sect. 3.6, resulting in an abundance $X\left(\mathrm{SO}_{2} / \mathrm{H}_{2}\right)=1.5 \times 10^{-6}$. The respective results are shown in Figs. D.1 and D.2.

For HCN(3-2), we applied the same threshold of $18 \sigma$ as for $\mathrm{SO}_{2}$ and fitted Gaussian components to the line profiles on each pixel, taking into account the hyperfine structure of the line. The velocity map and line widths displayed on Fig. D.3 are due to the intrinsic (thermal and rotational) broadening only, whereas the profile shown in Fig. D.4 represents the sum of the hyperfine components of $\mathrm{HCN}(3-2)$. As only one $\mathrm{HCN}$ rotational line is available, the HCN rotational temperature cannot be determined. We therefore fixed the rotation temperature at a value of $350 \mathrm{~K}$, the same temperature as we find from the $\mathrm{SO}_{2}$ modeling based on the similar emission region of $\mathrm{SO}_{2}$ and $\mathrm{HCN}$, see Figs. 17, B.1, and B.4. The $\mathrm{HCN}$ results are displayed in Fig. D.3. In particular, we derive an $\mathrm{HCN}$ column density of $\sim 1.6 \times 10^{15} \mathrm{~cm}^{-2}$, which translates to an $\mathrm{HCN}$ abundance of $X\left(\mathrm{HCN} / \mathrm{H}_{2}\right)=6.6 \times 10^{-7}$.

\footnotetext{
7 https://xclass.astro.uni-koeln.de
} 


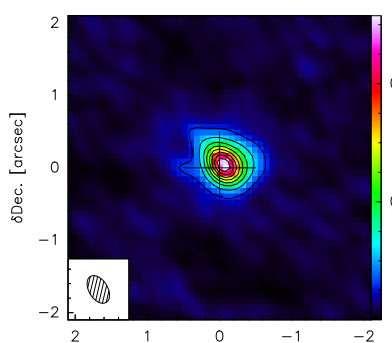

SR.A. $[\operatorname{arcsec}]$

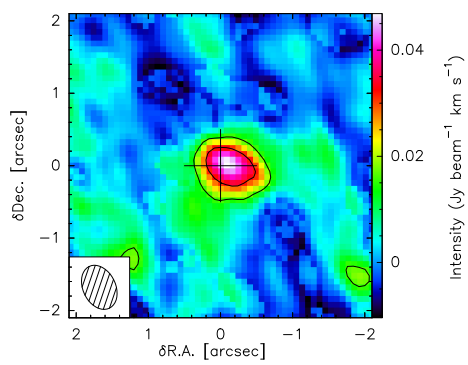

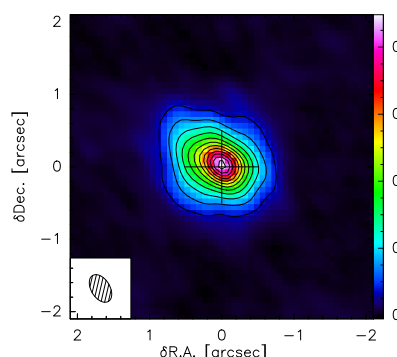

SR.A. [arcsec]

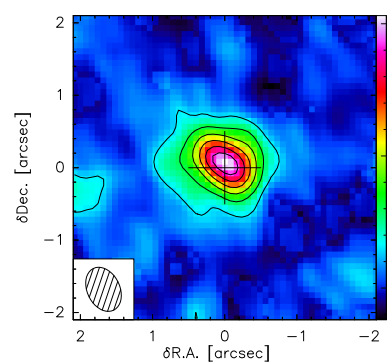

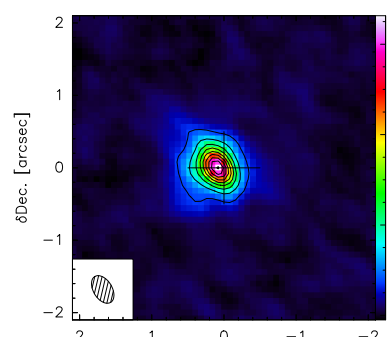

SR.A. [arcsec]

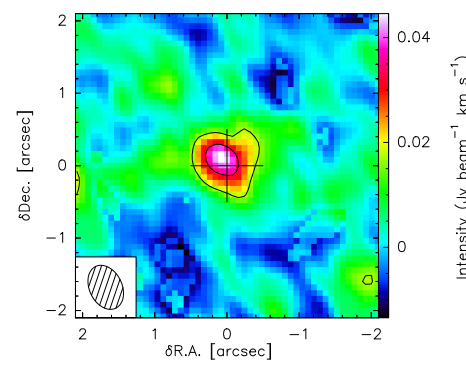

Fig. B.1. Velocity-integrated intensity maps of the $\mathrm{HCN}(3-2)$ (upper row) and $\mathrm{H}^{13} \mathrm{CN}(3-2)$ lines (lower row), covering three velocity intervals. Left: Blue line wing $\left[\mathrm{v}_{\mathrm{lsr}, *}-10, \mathrm{v}_{\mathrm{lsr}, *}-2\right] \mathrm{km} \mathrm{s}^{-1}$, Middle: line center $\left[\mathrm{v}_{\mathrm{ls}, *}-2, \mathrm{v}_{\mathrm{lsr}, *}+2\right] \mathrm{km} \mathrm{s}^{-1}$, Right: Red line wing $\left[\mathrm{v}_{\mathrm{lsr}, *}+2, \mathrm{v}_{\mathrm{lsr}, *}+10\right] \mathrm{km} \mathrm{s} \mathrm{s}^{-1}$. North is up and east is to the left. We note the different color scales. Contours are plotted every $10 \sigma$ for $\mathrm{HCN}$ and every $3 \sigma$ for $\mathrm{H}^{13} \mathrm{CN}$, where (from left to right) $1 \sigma=5.7,7.2,7.2 \mathrm{mJy} / \mathrm{beam} \mathrm{km} \mathrm{s}^{-1}$ for $\mathrm{HCN}(3-2)$ and $1 \sigma=5.2,5.0,5.6 \mathrm{mJy} / \mathrm{beam} \mathrm{km} \mathrm{s}{ }^{-1}$ for $\mathrm{H}^{13} \mathrm{CN}(3-2)$. The black ellipse in the lower left corner indicates the synthesized beam. We note that the $\mathrm{HCN}$ maps were produced using robust weighting, whereas for the $\mathrm{H}^{13} \mathrm{CN}$ maps, we applied natural weighting.
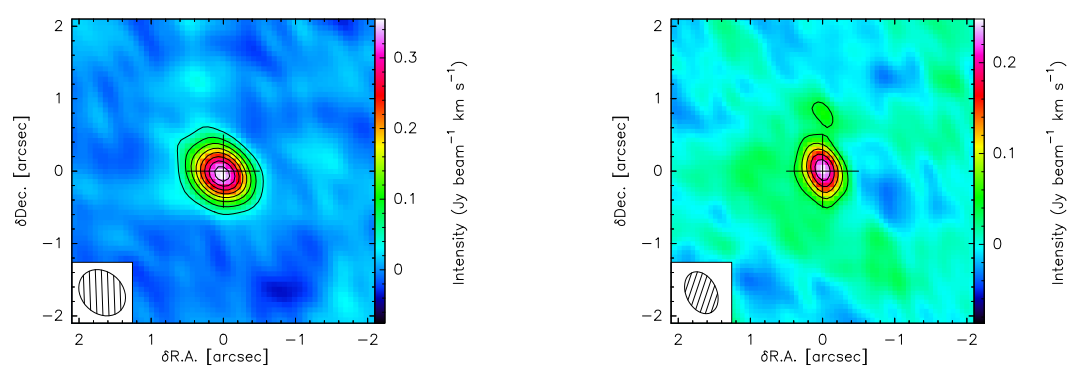

Fig. B.2. Zeroth moment maps of the $\mathrm{H}_{2} \mathrm{O} 232 \mathrm{GHz}$ (left) and $\mathrm{H}_{2} \mathrm{O} 263 \mathrm{GHz}$ (right) lines. North is up and east is to the left. We note the different color scales. Contours are plotted every $5 \sigma$ where $1 \sigma=13.8 \mathrm{mJy} / \mathrm{beam} \cdot \mathrm{km} \mathrm{s}^{-1}$ for the $232 \mathrm{GHz}$ line and $1 \sigma=13.92 \mathrm{mJy} / \mathrm{beam} \cdot \mathrm{km} \mathrm{s}{ }^{-1}$ for the $263 \mathrm{GHz}$ line. The black ellipse in the lower left corner indicates the synthesized beam. 


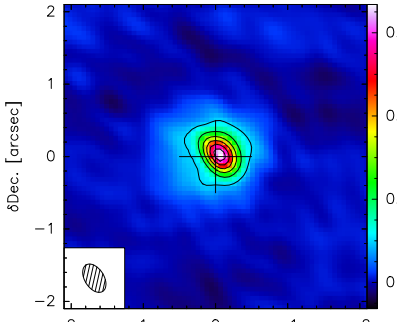

SR.A. [arcsec]
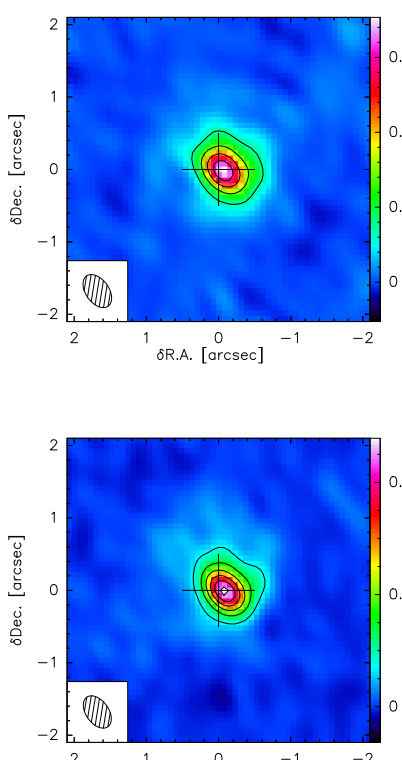

SR.A. [arcsec]

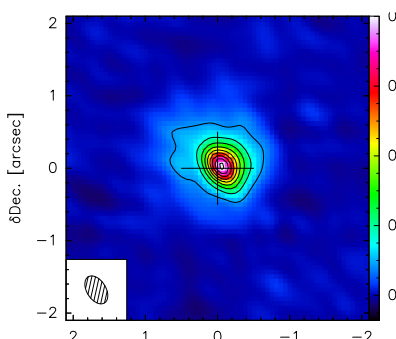

¿R.A. [arcsec]

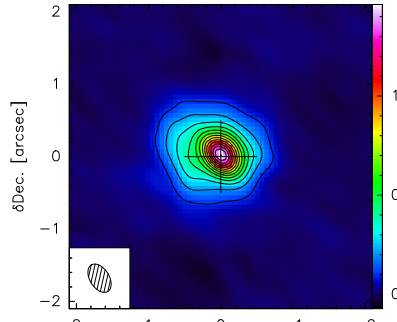

SR.A. [arcsec]

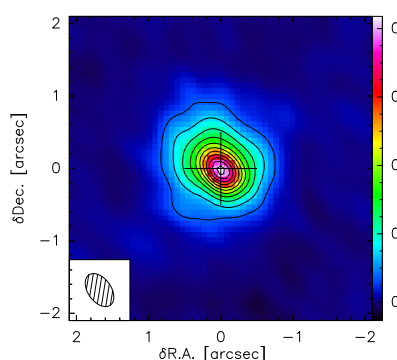

SR.A. $[\operatorname{arcsec}]$

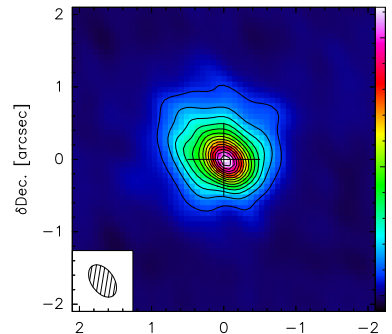

SR.A. [arcsec]

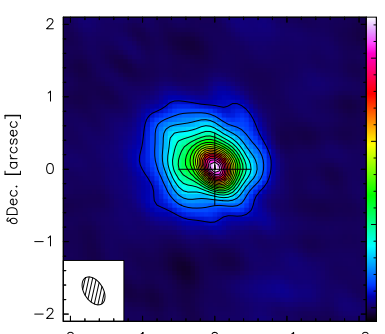

SR.A. [arcsec]

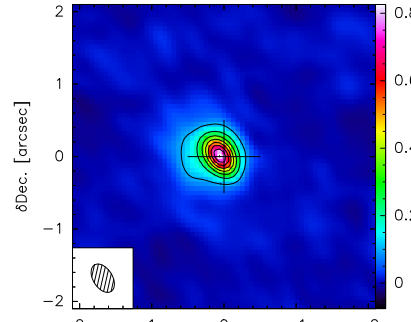

SR.A. [arcsec]

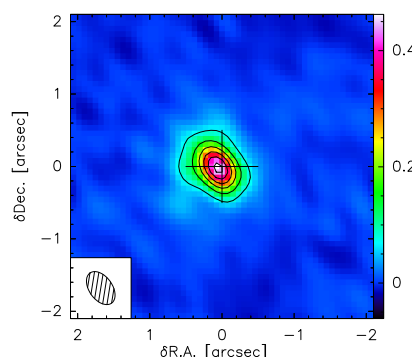

SR.A. [arcsec]

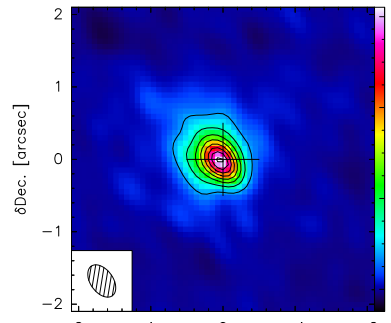

SR.A. [arcsec]

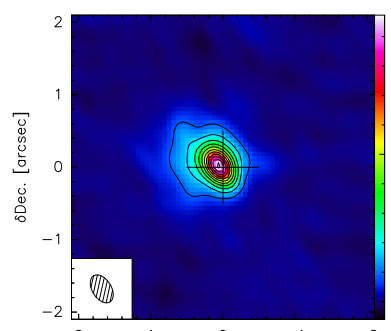

6R.A. [arcsec]

Fig. B.3. Velocity-integrated intensity maps of the $\mathrm{SO}(6(6)-5(5))$ (upper row), $\mathrm{SO}(5(5)-4(4))$ (second row), $\mathrm{SO}(6(5)-5(4))$ (third row) and $\mathrm{SO}(7(6)$ $6(5))$ (lower row) lines covering three velocity intervals. Left: Blue line wing $\left[\mathrm{v}_{\mathrm{lsr}, *}-10, \mathrm{v}_{\mathrm{lsr}, *}-2\right] \mathrm{km} \mathrm{s}^{-1}$, Middle: Line center [ $\left.\mathrm{v}_{\mathrm{lsr}, *}-2, \mathrm{v}_{\mathrm{lss}, *}+2\right]$ $\mathrm{km} \mathrm{s}^{-1}$, Right: Red line wing $\left[\mathrm{v}_{\mathrm{lsr}, *}+2, \mathrm{v}_{\mathrm{lsr}, *}+10\right] \mathrm{km} \mathrm{s}^{-1}$. North is up and east is to the left. We note the different color scales. Contours are plotted every $10 \sigma$, where (from left to right) $1 \sigma=10.0,10.4,10.3 \mathrm{mJy} / \mathrm{beam} \cdot \mathrm{km} \mathrm{s}^{-1}$ for $\mathrm{SO}\left(6(6)-5(5)\right.$ ), $1 \sigma=7.3,7.6,7.4 \mathrm{mJy} / \mathrm{beam} \cdot \mathrm{km} \mathrm{s}{ }^{-1}$ for $\mathrm{SO}(5(5)-4(4)), 1 \sigma=7.6,8.1,7.4 \mathrm{mJy} / \mathrm{beam} \cdot \mathrm{km} \mathrm{s}^{-1}$ for $\mathrm{SO}(6(5)-5(4))$, and $1 \sigma=8.7,9.6,8.7 \mathrm{mJy} / \mathrm{beam} \cdot \mathrm{km} \mathrm{s}^{-1}$ for $\mathrm{SO}(7(6)-6(5))$. The black ellipse in the lower left corner indicates the synthesized beam. 


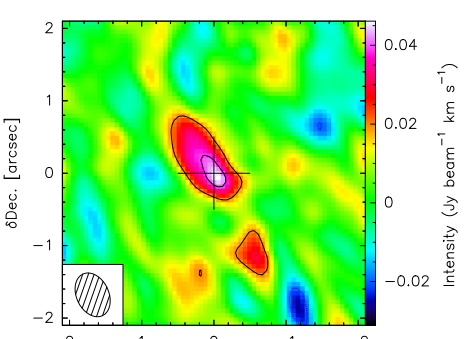

SR.A. [arcsec]
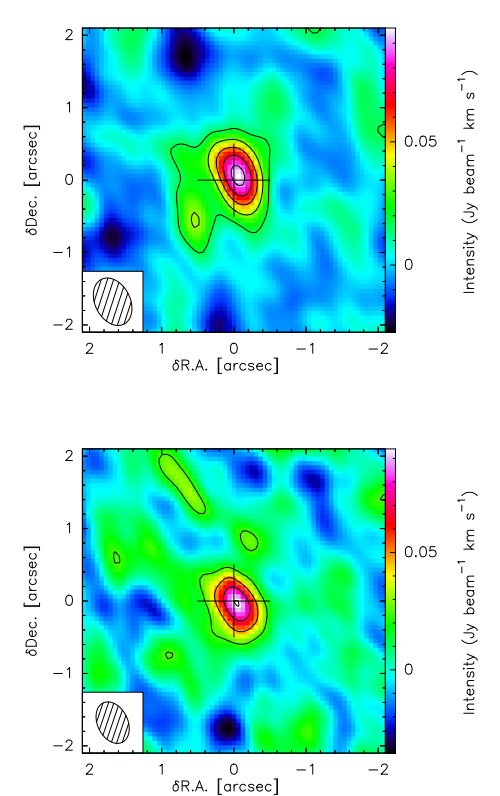
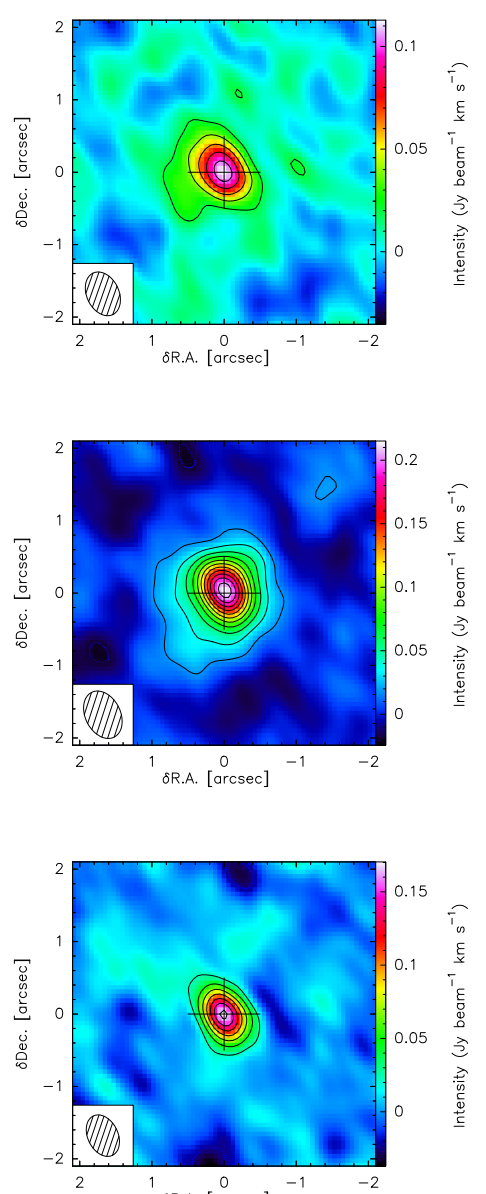
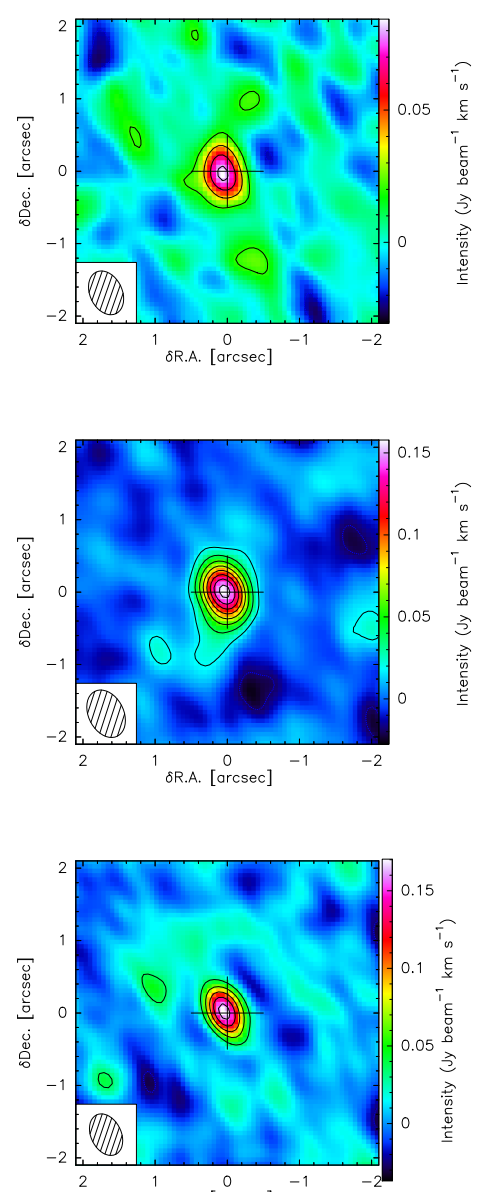

SR.A. [arcsec]

Fig. B.4. Velocity-integrated intensity maps of $\mathrm{SO}_{2}(9(3,7)-9(2,8))$ (lowest $\mathrm{E}_{u}$, upper row), $\mathrm{SO}_{2}(14(0,14)-13(1,13))$ (strongest $\mathrm{SO}_{2}$ line, second row), and $\mathrm{SO}_{2}$ (34(4,30)-34(3,31)) (highest $\mathrm{E}_{u}$, lower row), covering three velocity intervals. Left: Blue line wing [ $\left.\mathrm{v}_{\mathrm{lsr}, *}-10, \mathrm{v}_{\mathrm{lsr}, *}-2\right] \mathrm{km} \mathrm{s}{ }^{-1}$, Middle: line center $\left[\mathrm{v}_{\mathrm{lsr}, *}-2, \mathrm{v}_{\mathrm{lsr}, *}+2\right] \mathrm{km} \mathrm{s}^{-1}$, Right: Red line wing $\left[\mathrm{v}_{\mathrm{lsr}, *}+2, \mathrm{v}_{\mathrm{lsr}, *}+10\right] \mathrm{km} \mathrm{s}^{-1}$. North is up and east is to the left. We note the different color scales. Contours are plotted every $3 \sigma$, where (from left to right) $1 \sigma=6.5,6.7,6.6 \mathrm{mJy} / \mathrm{beam} \cdot \mathrm{km} \mathrm{s}^{-1}$ for SO $\mathrm{SO}_{2}(9(3,7)-9(2$, 8)) $1 \sigma=5.9,6.2,6.3 \mathrm{mJy} / \mathrm{beam} \cdot \mathrm{km} \mathrm{s}^{-1}$ for $\mathrm{SO}_{2}(14(0,14)-13(1,13)), 1 \sigma=7.8,7.8,7.7 \mathrm{mJy} / \mathrm{beam} \cdot \mathrm{km} \mathrm{s}^{-1}$ for $\mathrm{SO}_{2}(34(4,30)-34(3,31))$. The black ellipse in the lower left corner indicates the synthesized beam.

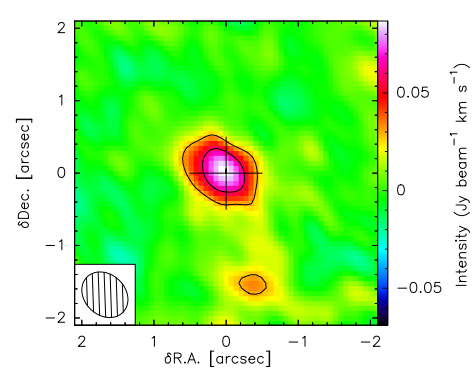

Fig. B.5. Zeroth moment map of the $\mathrm{PN}(\mathrm{N}=5-4, \mathrm{~J}=6-5)$ line. Contours are plotted every $3 \sigma$, where $1 \sigma=9.6 \mathrm{mJy} / \mathrm{beam} \mathrm{km} \mathrm{s} \mathrm{s}^{-1}$. North is up and east is to the left. The black ellipse in the lower left corner indicates the synthesized beam. 

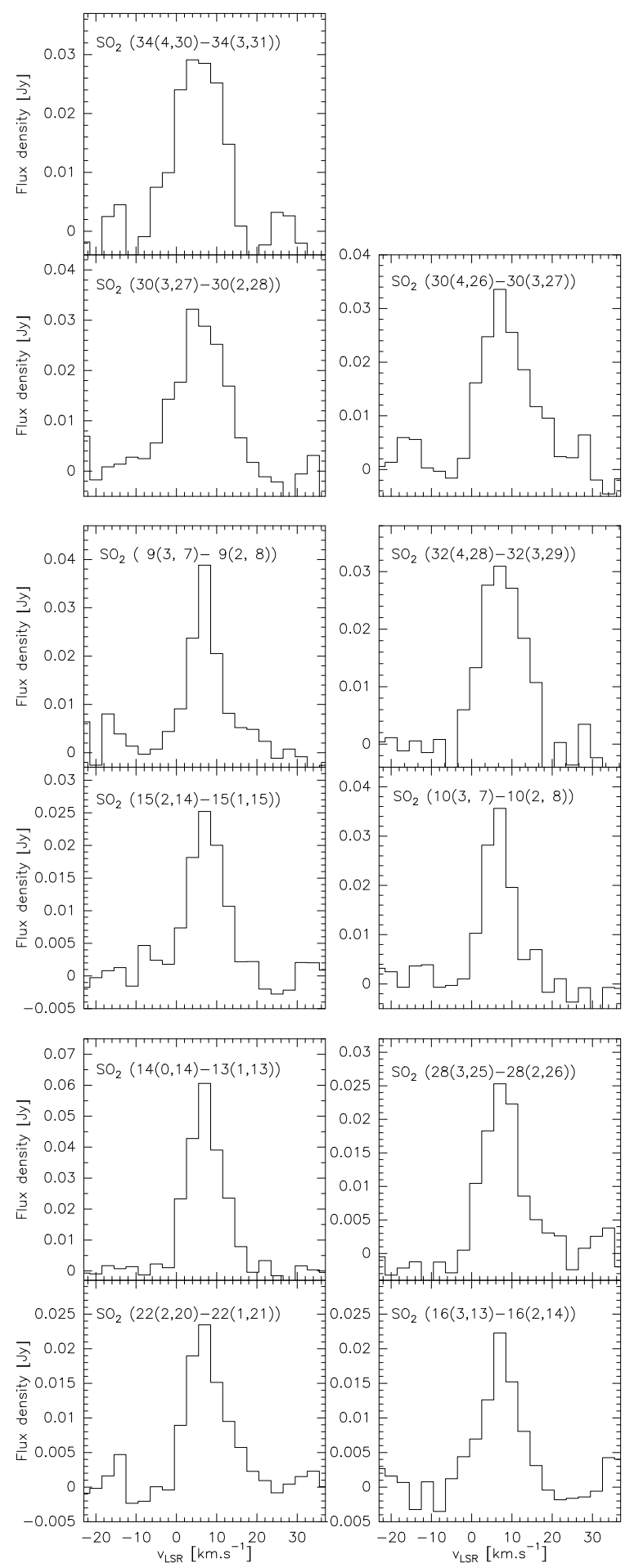

Fig. C.1. Profiles of all the 11 detected $\mathrm{SO}_{2}$ lines. A-configuration and D-configuration data are merged, the spectral resolution is $3 \mathrm{~km} \mathrm{~s}^{-1}$, and the emission is integrated over the central $2^{\prime \prime} \times 2^{\prime \prime}$ square aperture. The lines are ordered by decreasing frequency from top to bottom and left to right.
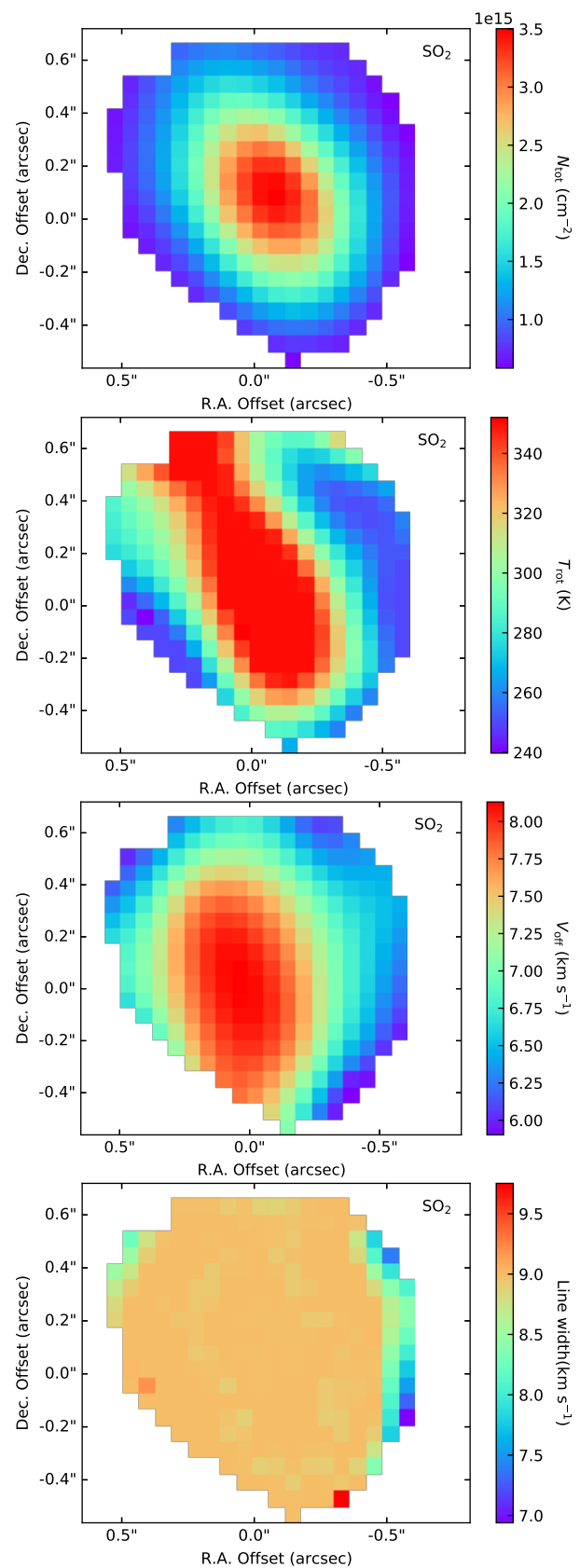

Fig. D.1. Maps of total column density, rotation temperature, velocity offset (in the LSR system), and line width for $\mathrm{SO}_{2}$ are derived with a threshold of $18 \sigma$ and fitting one Gaussian component to the line profiles. 
J. M. Winters et al.: Molecules, shocks, and disk in the axi-symmetric wind of the MS-type AGB star RS Cancri
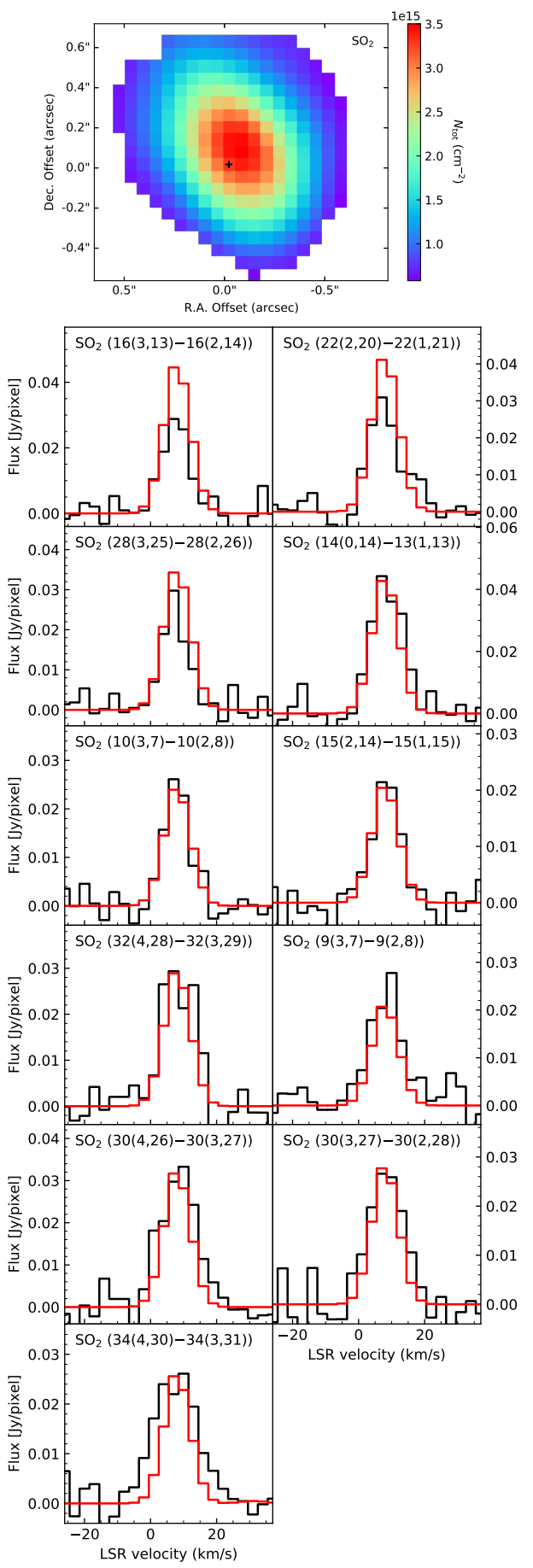

Fig. D.2. Profiles of the $11 \mathrm{SO}_{2}$ emission lines (in black) extracted on the central pixel and XCLASS modeled lines (in red) at the position of the continuum at RA $=09: 10: 38.77$ and $\mathrm{Dec}=+30: 57: 46.68$ (see Sect. 3.1) as marked on the upper diagram.
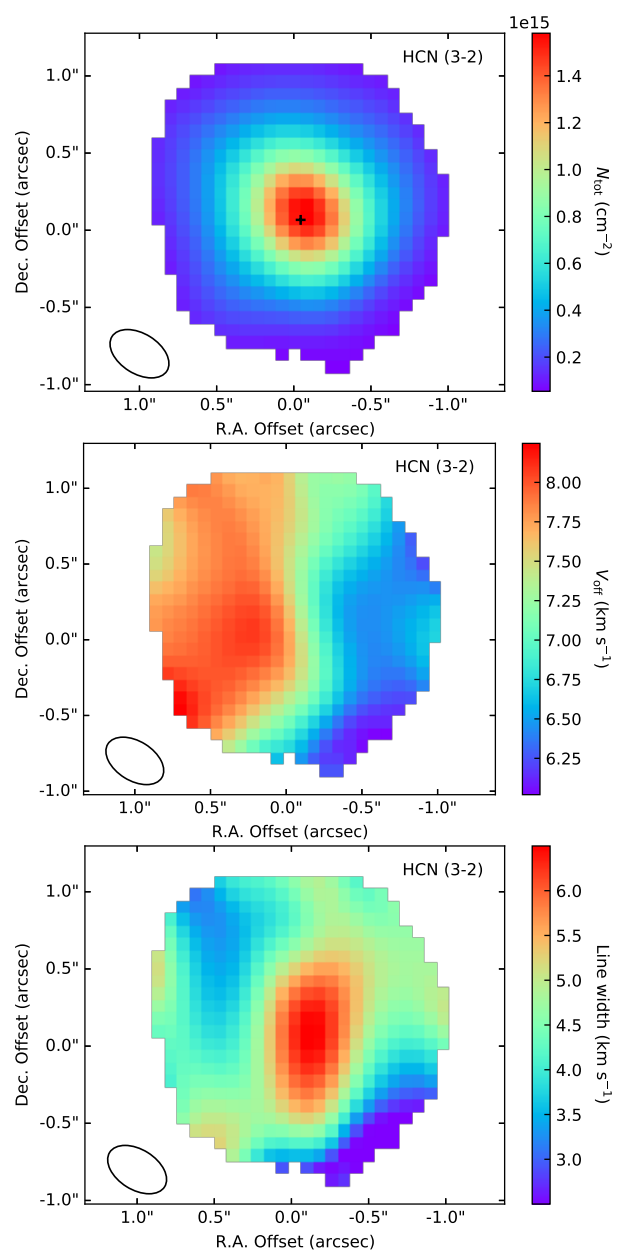

Fig. D.3. Maps of total column density, velocity offset $\left(=v_{\mathrm{lsr}}\right)$, and line width of $\mathrm{HCN}(3-2)$ (see text for details). The black ellipse in the lower left corner of each map indicates the synthesized beam.

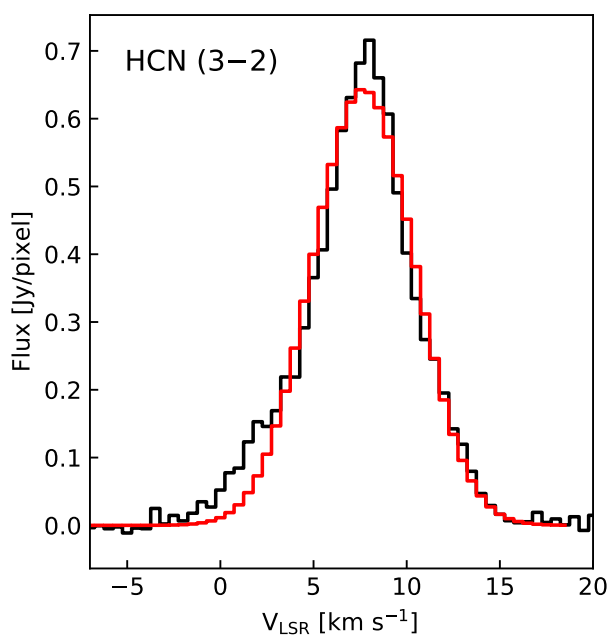

Fig. D.4. Profile of the $\mathrm{HCN}(3-2)$ emission line (in black) extracted on the central pixel and XCLASS modeled line (in red) at the position of the continuum at RA $=09: 10: 38.77$ and Dec $=+30: 57: 46.68$ (see Sect. 3.1) as marked on the upper diagram of Fig. D.3. 